\title{
Magnetic fields in isolated and interacting white dwarfs
}

\author{
Lilia Ferrario ${ }^{1}$ and Dayal Wickramasinghe ${ }^{2}$ \\ Mathematical Sciences Institute, The Australian National University, Canberra, ACT 2601, \\ Australia \\ Adela Kawka ${ }^{3}$ \\ International Centre for Radio Astronomy Research, Curtin University, Perth, WA 6102, \\ Australia
}

\begin{abstract}
The magnetic white dwarfs (MWDs) are found either isolated or in interacting binaries. The isolated MWDs divide into two groups: a high field group $\left(10^{5}-10^{9} \mathrm{G}\right)$ comprising some $13 \pm 4 \%$ of all white dwarfs (WDs), and a low field group $\left(B<10^{5} \mathrm{G}\right)$ whose incidence is currently under investigation. The situation may be similar in magnetic binaries because the bright accretion discs in low field systems hide the photosphere of their WDs thus preventing the study of their magnetic fields' strength and structure. Considerable research has been devoted to the vexed question on the origin of magnetic fields. One hypothesis is that WD magnetic fields are of fossil origin, that is, their progenitors are the magnetic main-sequence Ap/Bp stars and magnetic flux is conserved during their evolution. The other hypothesis is that magnetic fields arise from binary interaction, through differential rotation, during common envelope evolution. If the two stars merge the end product is a single high-field MWD. If close binaries survive and the primary develops a strong field, they may later evolve into the magnetic cataclysmic variables (MCVs). The recently discovered population of hot, carbon-rich WDs exhibiting an incidence of magnetism of up to about $70 \%$ and a variability from a few minutes to a couple of days may support the
\end{abstract}

\footnotetext{
${ }^{1}$ Lilia.Ferrario@anu.edu.au

${ }^{2}$ Dayal.Wickramasinghe@anu.edu.au
}

${ }^{3}$ Adela.Kawka@curtin.edu.au

Preprint submitted to Journal of ${ }^{A} T_{E} X$ Templates

January 29, 2020 
merging binary hypothesis. The fields in the weakly magnetic WDs may instead arise from a dynamo mechanism taking place in convective zones during post main-sequence evolution. Should this be the case, there may be a field strength below which all WDs are magnetic and thus fields are expected to always play a role in accretion processes in close binaries. Several studies have raised the possibility of the detection of planets around MWDs. Rocky planets may be discovered by the detection of anomalous atmospheric heating of the MWD when the unipolar inductor mechanism operates whilst large gaseous planets may reveal themselves through cyclotron emission from wind-driven accretion onto the MWD. Planetary remains have recently revealed themselves in the atmospheres of about $25 \%$ of WDs that are polluted by elements such as $\mathrm{Ca}, \mathrm{Si}$, and often also $\mathrm{Mg}, \mathrm{Fe}$, Na. This pollution has been explained by ongoing accretion of planetary debris. Interestingly, the incidence of magnetism is approximately $50 \%$ in cool, hydrogen-rich, polluted WDs, suggesting that these fields may be related to differential rotation induced by some super-Jupiter bodies that have plunged into the WD. The study of isolated and accreting MWDs is likely to continue to yield exciting discoveries for many years to come.

Keywords: Magnetic white dwarfs, magnetic cataclysmic variables, binary systems, Zeeman and cyclotron modelling, accretion processes, planetary systems.

\section{Introduction}

In recent years, there has been a rapid increase in the rate of discovery of MWDs. There are now around 600 isolated MWDs with magnetic fields spanning the range $10^{3} \mathrm{G}$ to $10^{9} \mathrm{G}$ and about 200 MWDs in interacting binaries. The sample is now large enough to allow studies of the group properties of the MWDs.

Early estimates of the space density of isolated MWDs were mostly based on those discovered by the Palomar Green (PG) survey which suggested an incidence of magnetism of only $\sim 2 \%$ for stars with fields greater than a few 
$10^{6} \mathrm{G}$. It was subsequently realised that this estimate should be revised upwards to at least $10 \%$ to account for the different volumes that are sampled by WDs with different masses when allowance is made for the higher mean mass of the MWDs (Liebert et al., 2003). A higher incidence of magnetism was also indicated from studies of the nearly complete $13 \mathrm{pc}(21 \pm 8 \%)$ and $20 \mathrm{pc}(13 \pm$ $4 \%$ ) volume limited samples of WDs conducted by Kawka et al. (2007). These findings were more recently confirmed by Holberg et al. (2016) who found that the inner $20 \mathrm{pc}$ volume contains $12 \%$ MWDs which translates to a space density of $\sim 0.6 \times 10^{-3} \mathrm{pc}^{-3}$.

Spectropolarimetric surveys have revealed the existence of low-field MWDs with fields in the range $10^{3}-10^{5} \mathrm{G}$ (e.g., Aznar Cuadrado et al., 2004; Jordan et al. 2007; Landstreet et al., 2012)). Their incidence is estimated by Landstreet et al. (2012) to be around $10 \%$, but this field regime still needs to be thoroughly investigated.

The origin of the magnetic fields of isolated and binary WDs has received renewed attention in recent years. The traditional view has been that their progenitors are the magnetic main-sequence $\mathrm{Ap}$ and $\mathrm{Bp}$ stars, and that the fields are essentially of fossil origin. A compelling argument in favour of this hypothesis is the correspondence that exists between the field strengths of the two groups under magnetic flux conservation (Ferrario and Wickramasinghe, 2005). However, given the incidence of magnetism among WDs the required birth rate of MWDs exceeds by a factor $\sim 2-3$ the birth rate of Ap and Bp stars (Kawka and Vennes, 2004). This and the lack of binaries consisting of a MWD paired with a fully detached, non-degenerate companion (Liebert et al., 2005, 2015; Tovmassian et al., 2016, 2018b, (a) has led to the suggestion that magnetic fields in WDs could be the outcome of binary interaction and stellar merging (see $\S[6)$.

It has been known for some time that MWDs tend to exhibit complex field structures and that off-centred dipole models generally produce a better fit to spectroscopic observations than centred dipole models (see Wickramasinghe and Ferrario, 2000). Structures with dominant quadrupolar components or evidence for signif- 
icant contributions from higher order multipoles have also emerged from detailed modelling of isolated and accreting MWDs (Maxted et al.,, 2000; Euchner et al., 2005, 2006; Beuermann et al., 2007; Landstreet et al., 2017), although not all MWDs require complex field structures to understand their spectra. This suggests that there may be a complex interplay between fossil and dynamo generated fields during stellar evolution and/or stellar merging events which are still poorly understood phenomena despite some extensive studies conducted over many decades (Tout et al., 2004; Brun et al., 2005; Featherstone et al., 2009; Potter and Tout, 2010; Quentin and Tout, 2018).

We begin by presenting an overview of the methods used for determining magnetic fields in WDs in $\S 2$ In this section we also present the rich variety of spectra that result from the Zeeman effect in WDs of different atmospheric compositions. The assumptions involved in the construction of model atmospheres for MWDs are presented in $\S 3$ where we also describe the methods that have been used to unravel the magnetic field structure of MWDs. The population properties of MWDs (fields, mass, temperature distributions) are highlighted in $\S$. The characteristics of interacting magnetic binaries can be found in $\S$ 罒 The population properties of isolated and accreting MWDs and current ideas on the origin of magnetic fields are presented and discussed in $\S 6$. A summary and an outlook for future research is presented in $\S[7$

\section{Measuring magnetic fields in WDs}

\subsection{Zeeman spectroscopy}

\subsubsection{Hydrogen lines}

Similarly to their non-magnetic counterparts, the vast majority of MWDs are hydrogen-rich and are classified as DAH, if the WD was originally discovered to be magnetic from Zeeman splitting, or DAP, if the WD was discovered to be magnetic from polarisation studies. An understanding of their properties relies heavily on the theory of the hydrogen atom at strong magnetic fields. 
The structure of the hydrogen atom at arbitrary field strengths was solved in the mid 1980's by various groups (Kemic, 1974; Roesner et al., 1984; Forster et al., 1984; Henry and O'Connell, 1985; Wunner et al., 1985) culminating in the publication of wavelengths and oscillator strengths of most of the important transitions in the ultraviolet, optical and infrared spectral regions as a function of the parameter

$$
\beta=\frac{\omega_{C}}{4 \omega_{R}}=\frac{B}{4.7 \times 10^{9} \mathrm{G}}
$$

that measures the relative importance of the cyclotron frequency of a free electron

$$
\omega_{C}=\frac{e B}{m_{e} c}
$$

to the Rydberg frequency $\omega_{R}$. Here $e$ is the electron charge, $m_{e}$ the electron rest mass and $c$ the speed of light. At low $\beta$, the atomic structure is dominated by the spherical symmetry of the Coulomb potential while at high $\beta$ is dominated by the cylindrical symmetry of the field, $B$.

The dependence of wavelength $\lambda$ on magnetic field, the so-called $\lambda-B$ curves, have formed the basis for all studies of MWDs. These calculations are for fields that are high enough for the magnetic splitting to be larger than the spin-orbit coupling (the Paschen-Back regime).

If we use the standard zero field quantum numbers $\left(n, l, m_{l}\right)$ to label energy levels ( $n$ is the principal quantum number, $l$ is the angular momentum quantum number, and $m_{l}$ is the magnetic quantum number), three basic Zeeman regimes can be identified: linear (removal of $m_{l}$ degeneracy), quadratic (removal of $l$ degeneracy), fully mixed (removal of $n$ degeneracy).

In the simplest Paschen-Back linear Zeeman regime $(\beta<<1)$, when the $m_{l}$ degeneracy is removed, all energy levels are shifted by an amount $\frac{1}{2} m_{l} h \omega_{C}$. A single line is split into a triplet composed of an unshifted central $\pi$ component $\left(\Delta m_{l}=0\right)$, a red-shifted $\sigma_{+}\left(\Delta m_{l}=+1\right)$ component and a blue-shifted $\sigma_{-}$ $\left(\Delta m_{l}=-1\right)$ component. The $\pi$ component occurs at the zero field frequency, $\omega_{0}$, while the two satellite $\sigma$ components occur at $\omega_{0}+\omega_{L}$ and $\omega_{0}-\omega_{L}$ where $\omega_{L}=$ $\omega_{C} / 2$ is the Larmor frequency. The $\pi$ components are linearly polarised, while 
the $\sigma_{-}$and the $\sigma_{+}$components are circularly polarised with opposite signs when viewed along the magnetic field. In the linear regime and in wavelength units the splitting between a $\sigma$ component and the unshifted central $\pi$ component is

$$
\delta \lambda_{L}=20.2\left(\frac{\lambda}{6564 \AA}\right)^{2}\left(\frac{B}{10^{6} \mathrm{G}}\right) \AA
$$

If the fields are low enough $\left(\sim 10^{6}-10^{7} \mathrm{G}\right)$ and the intrinsic broadening of the line due to pressure effects does not mask the Zeeman splitting, the characteristic pattern of a Zeeman triplet is detectable in flux spectra in the lower members of the Balmer and Lyman series at spectral resolutions typically used in WD surveys $(\approx 10 \AA)$. We show in Figure 1 the spectra of seven SDSS DAP WDs (Schmidt et al., 2003) showing the splitting of $\mathrm{H}_{\alpha}$ into a classical Zeeman triplet. At higher spectral resolutions, Zeeman triplet patterns can be detected in narrow non-LTE line cores in MWDs, extending the range of fields that can be measured by spectroscopy down to $\sim 10^{5}-10^{6}$ G. A nice set of data depicting how $\mathrm{H}_{\alpha}$ varies as the magnetic fields strength increases is the series of low field WDs shown in Fig.20btained by Landstreet et al. (2016). A powerful method for measuring magnetic fields, particularly in the very low field regime where the Zeeman splitting is small and unresolvable, is the use of circular spectropolarimetry across lines. A statistically significant detection of the expected change in the sign of circular polarisation across spectral lines allows such fields to be measured, and this is the method used to discover kilo-Gauss fields (see Figure 3 from Landstreet and Bagnulo (2019) and also §4.1).

In the next field regime the quadratic term dominates in the Hamiltonian so that the $l$ degeneracy is also removed although the field is still not strong enough for adjacent $n$ manifolds to overlap significantly $\left(\beta<10^{-3}\right.$ for Balmer lines). In this regime the $\pi$ components are also shifted. The quadratic effect is expected to be first seen at low fields as an asymmetry in the line profile and a blue-ward displacement of the centroid of the line from its zero field position and then at higher fields with the individual components resolved. Note that the quadratic effect becomes stronger as $n_{u}$ (upper principal quantum number) increases for a given $n_{l}$ (lower principal quantum number) - that is, it is relatively 


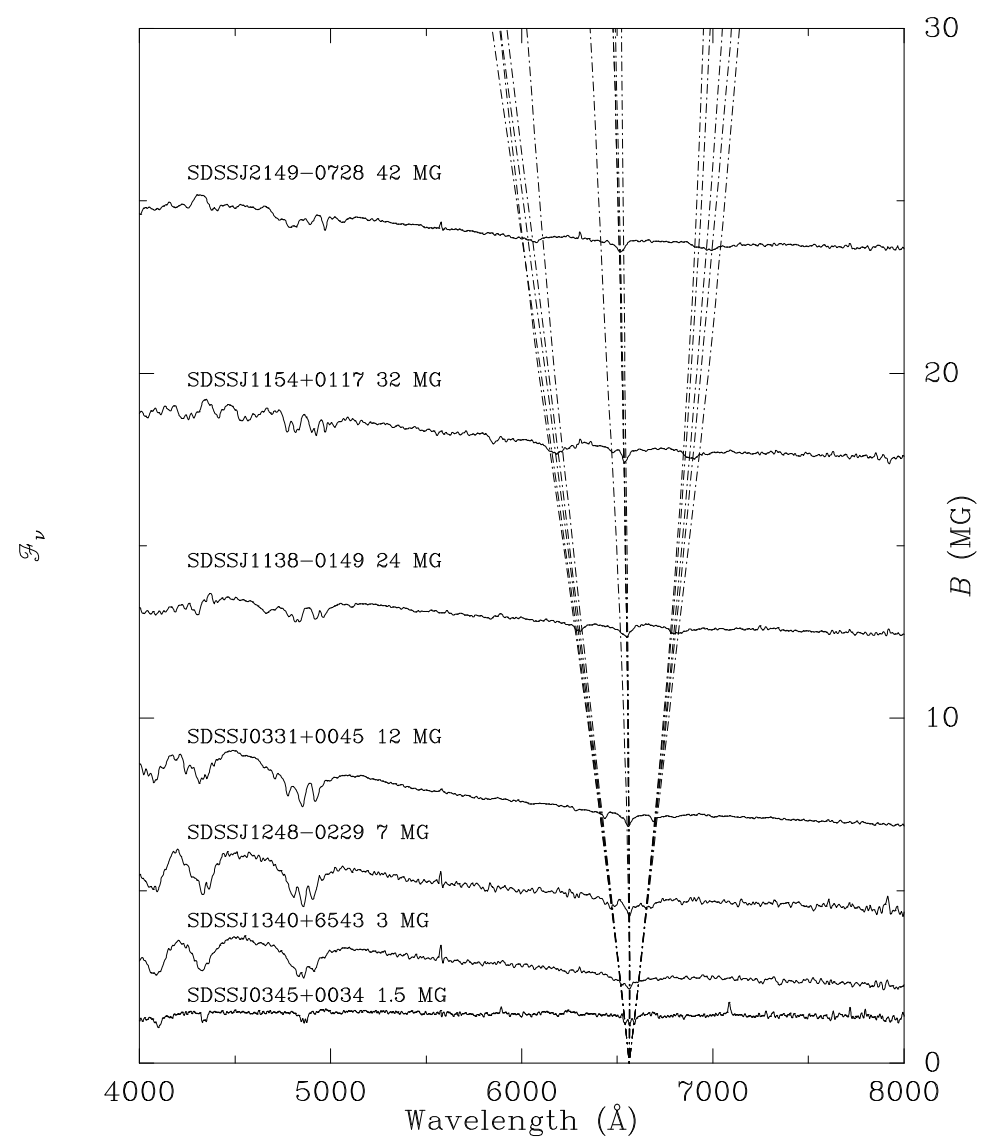

Figure 1: The Zeeman effect in the linear and quadratic regimes illustrated in the spectra of seven DAP WDs. The $\mathrm{H}_{\alpha}$ line is split into a triplet over the entire field range(1.5 $42 \mathrm{MG}$ ) while the quadratic Zeeman effect is already seen at $\mathrm{H}_{\beta}$ at a field of $12 \mathrm{MG}$ (from Schmidt et al., 2003). 


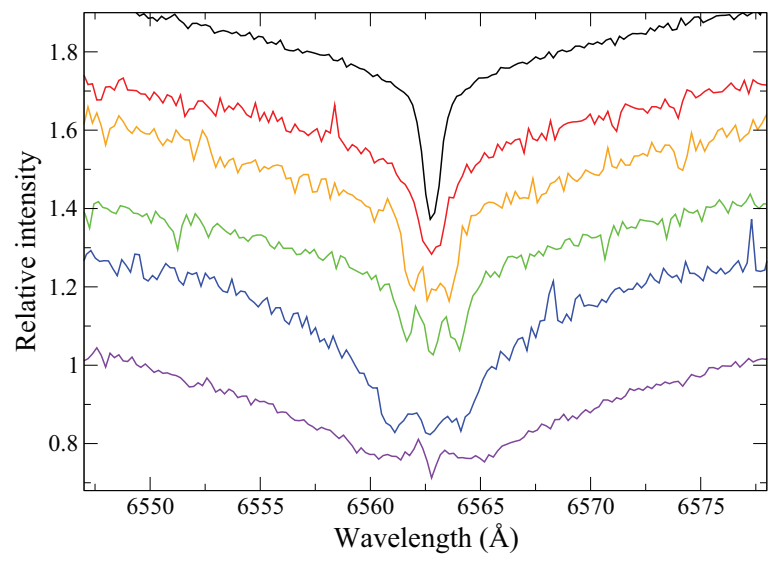

Figure 2: Intensity spectrum of a non-magnetic WD (top). The spectra that follow are those of MWDs with increasingly higher field strength. For full details see Landstreet et al. (2016).

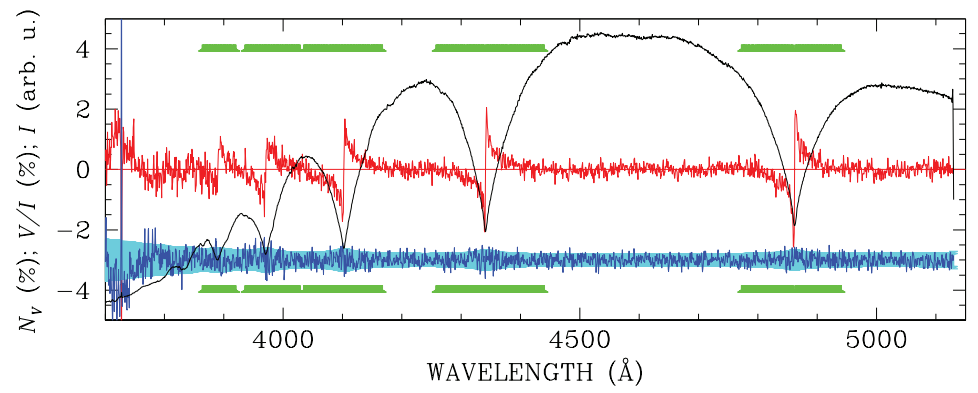

Figure 3: Spectropolarimetric observations of WD 1105-340. The black curve is the intensity profile and the red curve the circular polarisation $(\mathrm{V} / \mathrm{I})$ spectrum in percentage units (see Landstreet and Bagnulo, 2019, for full details). The change in the sign of circular polarisation across the members of the Balmer series is clearly visible. 
more important in the higher members of a given series as shown in Fig.11. The majority of MWDs are discovered through the detection of resolvable linear or quadratic Zeeman structures. At even higher fields $(\beta \sim 1$ - the diamagnetic

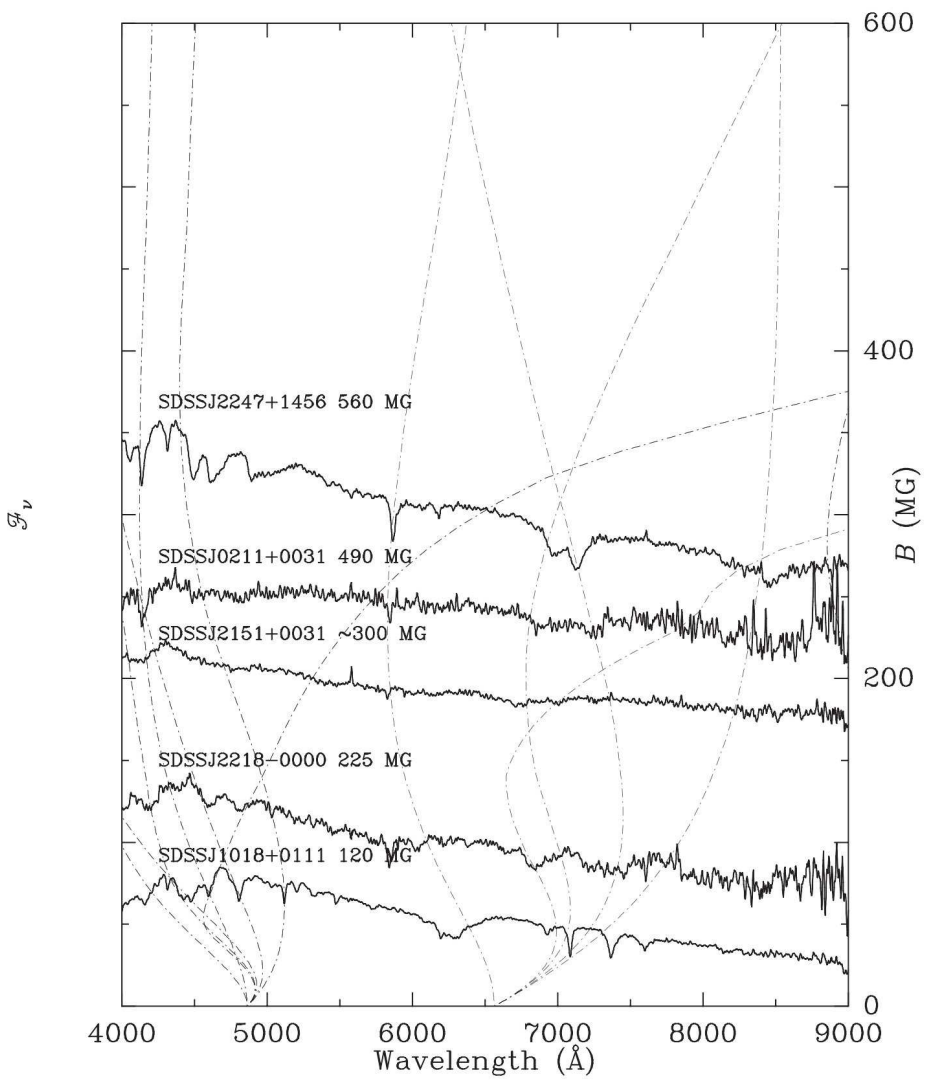

Figure 4: The spectra of five MWDs with fields between 120 and 560 MG showing stationary Zeeman components of $\mathrm{H}_{\alpha}$ and $\mathrm{H}_{\beta}$ (from Schmidt et al., 2003). The dash-dotted lines are the Balmer lines transitions as a function of magnetic field strength in MegaGauss. All the strong absorption features observed in these spectra are associated with transitions whose wavelengths are stationary (or nearly stationary) with magnetic field strength.

regime), different $n$ manifolds begin to overlap and the only good quantum numbers are the parity $\pi$ and $m_{l}$. Although the energy level diagram shows no simple structure at these fields, in the strong field regime $(\beta>>1)$ a new structure begins to appear, reflecting the quantisation of the motion of the 
electron perpendicular to the field into Landau energy states, with the motion along the field being effectively 1-D Coulombic. In the approach to this regime, the $\sigma^{+}$components become nearly "stationary" in the sense that large changes in the magnetic field result only in small changes in the wavelength. MWDs are discovered through the detection of these stationary components. In Figure 4 we present a montage of spectra for a range of field strengths showing the appearance of stationary components (Vanlandingham et al., 2005).

\subsubsection{Forbidden lines}
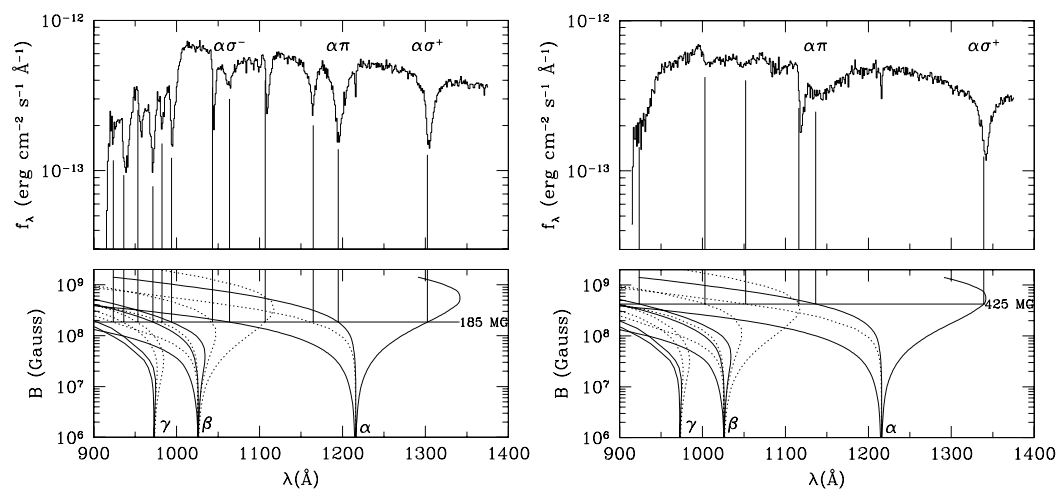

Figure 5: Top: Phase-resolved HST FOS/FUSE spectroscopy of RXJ 0317-853 at polarimetric phase $\phi=0$ (left) and $\phi=0.5$ (right). Bottom: the solid curves are the hydrogen Lyman transitions and the dotted curves the forbidden transitions that are enabled because of the strong electric fields induced by the super-strong magnetic fields. The observed spectra are compared to predicted line positions showing that the spectrum at $\phi=0$ has a field $B=$ $185 \mathrm{MG}$, while at $\phi=0.5$ has a field $B=425 \mathrm{MG}$ (from Vennes et al., 2003).

The UV spectra of both isolated and accreting MWDs have shown the presence of transitions that are normally forbidden. For example, the $1 s_{0}-2 s_{0}$ $\mathrm{Ly}_{\alpha}$ component that violates the parity/magnetic moment selection rules was detected by Burleigh et al. (1999) in the UV spectrum of the isolated HFMWD RXJ 0317-853 and by Gänsicke et al. (2001); Hoard et al. (2004) in the UV spectrum of the accreting MWD in the MCV AR UMa. Further observations of RXJ 0317-853 (see Fig. 5) conducted by Vennes et al. (2003) revealed the pres- 
ence of many more forbidden transitions (seven in total) comparable in strength to permitted transitions. Burleigh et al. (1999) have explained that such transitions become possible in the dense atmospheres of MWDs because free electrons and ions can induce Lorentzian electric fields as strong as $10^{9} \mathrm{~V} \mathrm{~m}^{-1}$ that act perpendicularly to the magnetic field. Without these electric fields, the magnetic quantum number and the $z$-parity $\pi_{z}$ are conserved. However, only the magnetic quantum number is conserved for parallel electric and magnetic fields while only the $z$-parity is conserved for perpendicular electric and magnetic fields. If fields are randomly oriented there is no discrete symmetry and thus additional dipole transitions, such as $1 s_{0}-2 s_{0}$, are enabled (Burleigh et al., 1999).

\subsubsection{Helium lines}

MWDs in which helium is the dominant element in their atmospheres, the DBP (or DBH) MWDs, are characterised by Zeeman split HeI lines in their high temperature spectra. The understanding of their spectra required the unravelling of the structure of the energy levels of the two electron He atom at arbitrary fields. Following early numerical calculations of the wavelengths and transition probabilities of the stronger HeI lines in the low field regime $(\beta<<1)$ (Kemic, 1974), detailed calculations carried out in the 1990's led to the determination of accurate energy levels of the low-lying states of HeI at arbitrary magnetic fields (Becken and Schmelcher, 2001; Al-Hujaj and Schmelcher, 2003) and references therein). The calculations cover the lowest five $(n \leq 5)$ singlet and triplet states for the subspaces $m=0, \pm 1, \pm 2, \pm 3$ and include transition probabilities for the important optical and UV transitions. The lines split into triplets in the lowfield regime, and stationary components appear as for hydrogen at high fields (see Jordan et al., 2001, and Figure 6).

Zeeman split HeI lines have been identified in a sample of MWDs with fields in the range $4 \times 10^{6}-6 \times 10^{8} \mathrm{G}$ including the strongly polarised MWD GD 229 (Jordan et al., 1998; Wickramasinghe et al., 2002). A comparison of the spectra of GD 229, HE 1211-1707, and HE 1043-0502 with HeI Zeeman mod- 
els constructed under various simplifying approximations is shown in Figure 6 (Wickramasinghe et al., 2002). The identifications with HeI lines are secure except in the case of HE 1043-0502 where other interpretations may be possible. The discrepancies between observations and theory in GD 229 may be caused by resonances in the HeI bound-free opacities for which there is at present no adequate theory (see $₫ 3$ ).

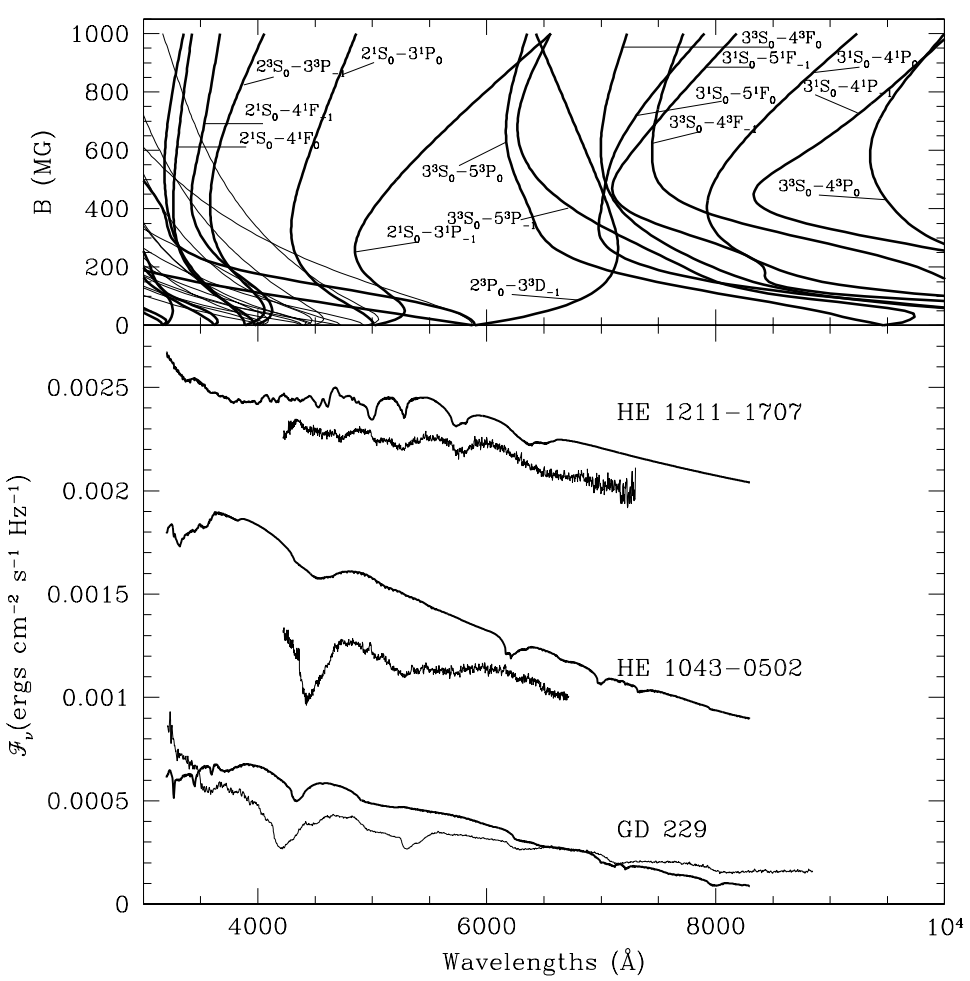

Figure 6: Top panel: Helium transitions as a function of magnetic field strength in MegaGauss. Bottom panel: A comparison of centred dipole models for helium rich WDs with observations of GD 229, HE 1043-0502, and HE 1211-1707. The models have, respectively, polar fields $B_{d}=$ 520, 820, and $50 \mathrm{MG}$ (from Wickramasinghe et al., 2002).

\subsubsection{Carbon molecular bands}

A sub-class of cool WDs $\left(T_{\text {eff }}<8,000 \mathrm{~K}\right)$ have atmospheres that (i) are He dominated, (ii) are $\mathrm{H}$ deficient, and (iii) exhibit molecular bands of $\mathrm{C}_{2}$ 
that increase in strength as the temperature decreases. Occasionally they also show $\mathrm{CH}$ and atomic $\mathrm{C}$ lines. These WDs were first noted in the late 50's (Greenstein and Matthews, 1957) and were later named "DQ" WDs (Greenstein, 1984). They are too cool to allow the formation of neutral He lines and any trace of pollution reveals itself easily due to the high pressure and low continuum opacity of helium-dominated regions. The presence of carbon in these cool DQ WDs is probably caused by the dredge-up from the core by the helium convection regions as first proposed by Pelletier et al. (1986).

Spectropolarimetric observations have shown that a few of these cool DQ WDs are strongly magnetic (Liebert et al., 1978). More recent observations have revealed field strengths in the range $\sim 7-100 \mathrm{MG}$ (Vornanen et al., 2010, 2013, and Kawka et al. 2019, in preparation). We show in Fig.7three WDs showing

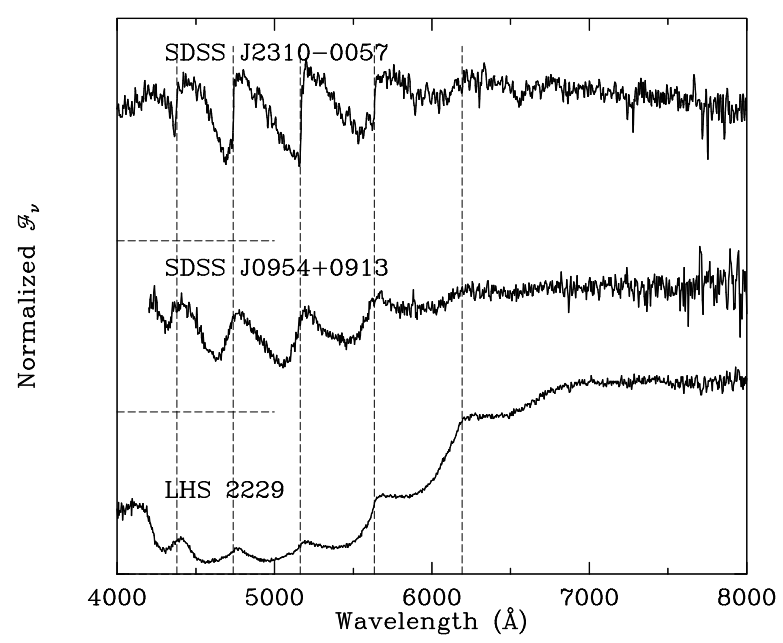

Figure 7: Comparison between SDSS J0954+0913, the normal ( $\mathrm{C}_{2}$ Swan band) molecular WD SDSS J2310 0057, and the peculiar magnetic DQ star LHS 2229. The horizontal dashed lines indicate the zero-flux levels while the vertical dashed lines indicate the band heads of normal $\mathrm{C}_{2}$ Swan features (from Vanlandingham et al., 2005).

the distortion of the characteristic $\mathrm{C}_{2}$ Swan bands with increasing magnetic field. The WD LHS 2229 has a higher circular polarisation than SDSS J0954+0913 while SDSS J2310-0057 is unpolarised and non-magnetic. We note that Kowalski 
(2010) showed that the blue shifted $\mathrm{C}_{2}$ molecular bands can be explained by the high pressure that exists in very cool WDs. Therefore, these shifted Swan molecular bands are most likely $\mathrm{C}_{2}$ molecular bands that have been pressure shifted.

Field estimates of DQ MWDs have so far relied on theoretical estimates of the quadratic Zeeman effect on $\mathrm{C}_{2}$ and $\mathrm{CH}$ bands based on the simplified rigid rotator model of Bues and Pragal (1987). More detailed theoretical calculations of the Zeeman effect in the Paschen Back regime are available but have so far only been used to model the intensity and polarisation spectrum of G9937 indicating a field of $8 \mathrm{MG}$ (Berdyugina et al., 2007). Models for the more strongly polarised and presumably higher field WDs, such as LHS 2229, are still unavailable.

\subsubsection{Metal lines}

WDs have such a strong surface gravity that the diffusion timescale for metals to sink to the bottom of the atmospheres is very short (days to weeks for a hydrogen-dominated atmosphere, as shown by Koester and Wilken, 2006). Nonetheless, some $25 \%$ of WDs show traces of metals in their spectra (generally $\mathrm{Ca}$ and $\mathrm{Si}$, but also Mg, Fe, Na and others, (Zuckerman et al., 2003, 2011; Farihi et al., 2011; Kawka and Vennes, 2016; Farihi et al., 2016). We shall come back to these intriguing objects in $\$ 4.5$. The level of an electron in an atom can be described by the three quantum numbers, $J, L, S$, where $J$ is the total angular momentum, $L$ is the orbital angular momentum and $S$ is the spin angular momentum. When a metal atom is placed in a magnetic field its levels are split into $2 J+1$ components that are defined by the magnetic quantum number $m=-J,-J+1, \cdots, J-1, J$. The resulting shifted Zeeman lines can be calculated using:

$$
\Delta \lambda=\frac{e B \lambda^{2}}{4 \pi m_{e} c^{2}}\left(g_{l} m_{l}-g_{u} m_{u}\right) \approx 4.67 \times 10^{-7} \lambda^{2} B\left(g_{l} m_{l}-g_{u} m_{u}\right)
$$

The magnetic quantum numbers of the upper and lower levels are $m_{u}$ and $m_{l}$, respectively. Just like for the hydrogen atom, the permitted transitions that are 
allowed are $\Delta m=0, \pm 1$. Similarly, the Lánde factors of the upper and lower levels are $g_{u}$ and $g_{l}$, respectively. Lánde factors for light elements, which includes calcium, sodium and magnesium, can be calculated assuming LS coupling:

$$
g=1+\frac{J(J+1)-L(L-1)+S(S+1)}{2 J(J+1)},
$$

In heavier atoms, such as iron, the interaction between the orbital and spin angular momenta is as strong as the interaction between individual spins or orbital angular momenta and LS coupling is no longer valid. Lánde factors for heavier elements can be obtained from the Vienna Atomic Line Database (VALD)4

The relative intensities of the Zeeman components in metal lines is proportional to functions of the total angular momentum and the magnetic quantum number (Condon and Shortley, 1963). For transitions where $\Delta J=0$ and $\Delta m=0$

$$
I \propto m^{2}
$$

and for $\Delta m= \pm 1$

$$
I \propto \frac{1}{4}(J \mp m)(J \mp m+1) .
$$

For transitions where $J \rightarrow J+1$ and $\Delta m=0$

$$
I \propto(J+1)^{2}-m^{2}
$$

and for $\Delta m= \pm 1$

$$
I \propto \frac{1}{4}(J \pm m+1)(J \pm m+2) .
$$

Finally, for transitions where $J \rightarrow J-1$ and $\Delta m=0$

$$
I \propto J^{2}-m^{2}
$$

and for $\Delta m= \pm 1$

$$
I \propto \frac{1}{4}(J \mp m)(J \mp m-1) .
$$

\footnotetext{
${ }^{4}$ http://vald.astro.univie.ac.at/ vald3/php/vald.php (Kupka et al., 2000).
} 
Table 1 in Appendix A lists three different angular momenta, Lánde factors and relative intensities of the Zeeman components for the most commonly detected element lines in WD atmospheres. Fig.8 shows the spectrum of NLTT 7547, a cool DAZ WD, compared to model spectra calculated at $B_{d}=240 \mathrm{kG}$. The different strengths of the multiple Zeeman components of the metal lines are clearly noticeable.

The regime described above is valid for heavy elements in relatively low magnetic fields. In higher magnetic fields, the Paschen-Back regime becomes appropriate and the Zeeman split lines become triplets. For Na, this occurs for $B \gtrsim 100 \mathrm{kG}$ and for others such as $\mathrm{Mg}$ and $\mathrm{Ca}$, this occurs for $B \gtrsim 2 \mathrm{MG}$.
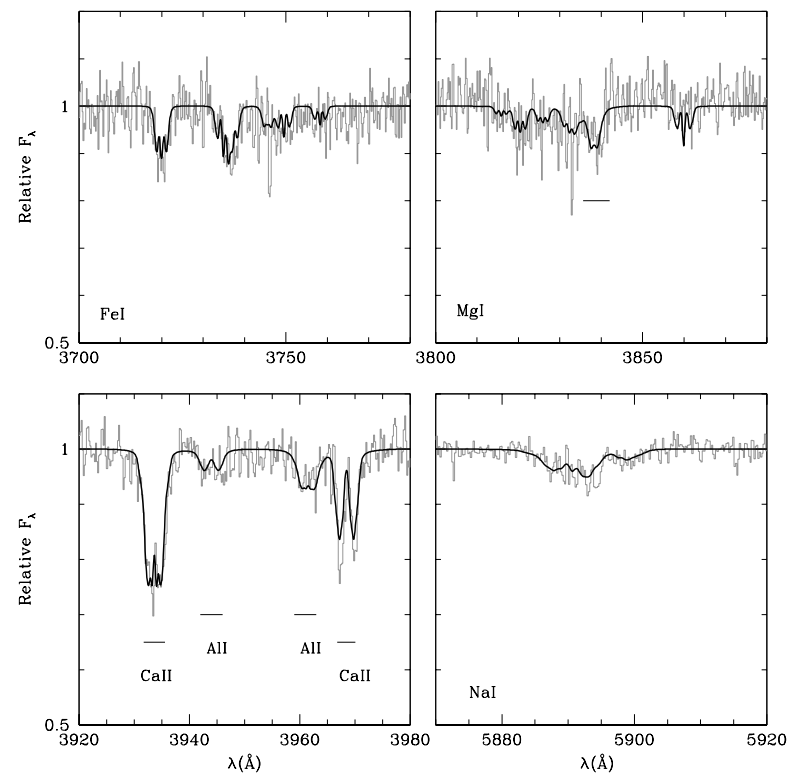

Figure 8: Spectrum of the cool DAZ NLTT 7547 (grey) compared to the best fitting model (black) at $T_{\text {eff }}=5460 \mathrm{~K}, \log g=8.04$ and $B_{d}=240 \mathrm{kG}$. The Zeeman split lines of sodium, magnesium, calcium and iron are shown. (see Kawka et al., 2019) for details. 


\subsection{Cyclotron lines and magnetised winds}

Free electrons in the atmospheres of MWDs are expected to give rise to thermal cyclotron radiation which results in an absorption feature at the cyclotron fundamental $\omega_{C}$ which corresponds to a wavelength

$$
\lambda_{c}=\frac{2 \pi}{\omega_{C}}=10710\left(\frac{10^{8} \mathrm{G}}{B}\right) \AA
$$

Although the integrated cross-section is similar to that for bound-free $\mathrm{H}$ opacity, the line is intrinsically narrow with a thermal width

$$
\delta \omega_{C} / \omega_{C}=\sqrt{2 k T / m_{e} c^{2}}|\cos \psi|
$$

where $\psi$ is the angle between the field direction and the line of sight (Martin and Wickramasinghe, 1979). The cyclotron fundamental should in principle be detectable in the optical to near IR band in MWDs with fields of a few $10^{8} \mathrm{G}$.

So far there has been no clear evidence for such a feature in the spectra of high-field MWDs, although the broad absorption feature extending over $\sim 1,000 \AA$ in the optical band in the strongly polarised MWD G240-72 may be of cyclotron origin (Martin and Wickramasinghe, 1979). In general, the detection of cyclotron features requires a very nearly uniform field structure over the visible surface because magnetic field broadening (with $\lambda \propto B$ ) is very effective in rendering the feature undetectable (see Martin and Wickramasinghe, 1979). Given the complex field structures that are now found in MWDs, and the possibility that the visible surface may present a nearly uniform field at some magnetic phases, one may expect that the cyclotron feature will be unambiguously detected in the absorption spectra of some MWDs in the near future.

Another method of detecting the cyclotron resonance is by using polarimetry. Cyclotron emission is 100\% circularly polarised when viewed along the field and elliptically polarised at a general viewing angle. The polarisability of the plasma and the index of refraction changes across the cyclotron resonance so as to give rise to a rotation in the polarisation angle by $90^{\circ}$ (or less than $90^{\circ}$ if vacuum 
polarisation is important, see Zheleznyakov and Serber, 1991). Spectropolarimetric data of Grw $+70^{\circ} 8247$ do show such a rotation near $5500 \AA$ (West, 1989), although such rotations may also be caused by continuum absorption edges.

The intriguing possibility that radiation pressure acting on the cyclotron resonance may drive outflows in high-field MWDs has been discussed by Zheleznyakov and Serber (1991). The mass loss rates are estimated to be $\sim(0.3-1) \times 10^{-13} \mathrm{M}_{\odot} \mathrm{yr}^{-1}$ for MWDs with effective temperatures $(2-5) \times 10^{4} \mathrm{~K}$ and magnetic fields of $(1.5-10) \times 10^{8} \mathrm{G}$. At the low densities expected in such coronal outflows, vacuum polarisation could have a significant effect across the vacuum resonance where the polarisability of the plasma and the vacuum cancel (Gnedin et al., 2006; Zheleznvakov and Serber, 1991). The vacuum resonance occurs at $\lambda \propto B / N_{e}$ and in the context of WDs, will be important in the optical band at the low electron densities $N_{e} \leq 10^{8} \mathrm{~cm}^{-3}$ that may be expected in such outflows.

\subsection{Constraining gravity theories using gravitational birefringence}

Some non-standard theories of gravity that go beyond general relativity by coupling the electromagnetic field directly with gravitational gauge fields could be constrained by studies of continuum polarised emission from MWDs (Solanki et al., 1999). For certain types of coupling, space-time becomes birefringent (gravitational birefringence) causing a phase-shift between different polarisation components leading to an additional source of depolarisation over and above the depolarisation that occurs due to the magnetic bi-refringence of the plasma as the radiation propagates through the atmosphere. The predicted phase-shift is directly proportional to the coupling constant and the gravity at the surface of the star and inversely proportional to the wavelength of observation. A measurement of the excess depolarisation of polarised radiation emitted by an astronomical source can in principle be used to constrain such theories. The observed levels of polarisation in the MWDs Grw $+70^{\circ} 8247$ (Solanki et al., 1999) and REJ0317-853 (Preuss et al., 2004) have been used to place some limits on the coupling constant for specific non-metric gravity models. These limits could be significantly improved when we have a better understanding of 
the atmospheres of MWD atmospheres and their intrinsic polarised emission. Potentially, neutron stars with their stronger surface gravities would be better suited for constraining such theories.

\section{Model atmospheres and magnetic field structure}

\subsection{Model atmospheres}

Calculations of model atmospheres for MWDs must include the effects of the magnetic field on continuum and line opacities and incorporate a full solution of the polarised transfer equations in the four Stokes parameters $\{I, Q, U, V\}$ that allows for the mixing of the different modes of propagation due to magnetooptical effects (Martin and Wickramasinghe, 1979). Magnetic fields may also have a direct influence on the hydrostatic equilibrium of the atmosphere. However, there are no calculations at present that fully account for all of these effects so our understanding of the atmospheres of MWDs is still incomplete.

\subsubsection{The force-free approximation and hydrostatic equilibrium}

The possible effect that a magnetic field may have on the hydrostatic and thermal equilibrium of a star has been discussed by several investigators (e.g. Landstreet, 1987; Fendt and Dravins, 2000). It is generally assumed that the magnetic field in the atmosphere is force-free maintained by currents in the interior of the WD. In this approximation, the magnetic pressure $\left(B^{2} /(8 \pi)\right)$ balances the magnetic tension along field lines $(\vec{B} \cdot \nabla \vec{B})$ so that there is no net magnetic force, even though each of these forces exceeds the gas pressure in the atmosphere by many orders of magnitude.

It has been argued that the Ohmic decay of a fossil field would lead naturally to a decay-induced Lorentz force that would drive a meridional motion perpendicular to field lines and affect the magneto-hydrodynamic structure of the WD atmosphere (Landstreet, 1987; Jordan, 1992). Non force-free configurations are also possible if the electric currents that maintain the field in the interior of the star also extend to the atmosphere (Fendt and Dravins, 2000). 
It has been estimated that a $\sim 2-10 \mathrm{MG}$ toroidal field that is superimposed on a $100 \mathrm{MG}$ poloidal field and maintained by current flows would lead to an increase in the scale height of the atmosphere by a factor $\sim 10$. Such a change in the atmospheric structure should in principle be detectable from line profiles (see Friedrich et al., 1994).

\subsubsection{Magnetic dichroism and birefringence}

Field dependent opacities (magnetic dichroism) and the associated magnetooptical parameters (magnetic birefringence) can be calculated for bound-bound transitions of hydrogen or neutral helium using existing calculations of energy levels and transition probabilities. As we have seen, the bound states of $\mathrm{H}$ and He show a rich and complex structure which must translate into complex structure in the bound-free opacities. A full quantum mechanical treatment of bound-free transitions must allow for the quantisation of the continuum into quasi-Landau levels in the presence of the Coulomb potential. The first results of such calculations for the hydrogen atom show how complex resonances develop in the Landau continua (Merani et al., 1995). Attempts at using these results to model the flux and polarisation spectra of Grw $+70^{\circ} 8247$, however, have met with limited success particularly with regard to polarisation. When the H bound-free opacities calculated by Zhao and Stancil (2006) and covering a wider field range are supplemented with associated magneto-optical parameters they should lead to the construction of better models of the hotter magnetic DA WDs. At present, though, there are no similar calculations for the helium atom, which are important for the modelling of the magnetic DB WDs. Nor are there calculations of the field dependence of $\mathrm{H}^{-}$or $\mathrm{He}^{-}$opacity which are important in cool WDs.

Although bound-free calculations predict narrow resonances in the Landau continua, there has been no observational evidence for such features in the spectra of MWDs. As with the cyclotron resonance, because of magnetic field broadening, the detection of such features would require a field structure which is very nearly uniform over the visible stellar surface. 
The model atmospheres that are currently in use for studying MWDs have adopted various approximations to the continuum opacity. These range from a simple linear splitting and shifting of ionisation edges, to a splitting and shifting of edges which allow transitions from generally Zeeman-split bound states to discrete continuum states assumed to be the Landau's states of a free electron (Jordan, 1992) for the case of hydrogen (see Wickramasinghe and Ferrario, 2000, for more details). Such approximations have proven to be adequate for establishing or constraining the magnetic field structure over the stellar surface where the dominant effect is the Zeeman splitting and shifting of the lines as a function of field strength (3.2).

\subsection{Magnetic field broadening and magnetic field structure}

The wavelength of a Zeeman component is strongly field dependent in most Zeeman regimes except for those components that become "stationary" in the high-field regime (see section 2 and Fig.4). In addition to pressure broadening, the spectral lines are also broadened by field spread across the visible stellar surface. In the simplest case of a dipolar field distribution and a component which shifts entirely according to the linear Zeeman effect, magnetic field broadening will impart to the line an intrinsic width of $\approx 2 \lambda_{L}$, where $\lambda_{L}$ is the Larmor wavelength (see $\S 2.1 .1$ ). For typical field strengths of $\approx 10^{7} \mathrm{G}$, and WD temperatures of $\approx 10,000 \mathrm{~K}$, this width exceeds the pressure or thermal width of a line $(\approx 10 \AA)$ by an order of magnitude, and thus magnetic field broadening dominates. Stationary components can therefore be used to investigate field structure even in the absence of a detailed atmospheric structure.

Rotating WDs provide the best opportunity for investigating the underlying field structure since they allow different parts of the stellar surface to be viewed at different rotational (and generally magnetic) phases. The Zeeman shift of a line component depends only on the magnitude of the magnetic field, while the circular and linear polarisation of a Zeeman split component also carry information on the field direction. Phase-dependent spectropolarimetric observations therefore provide strong constraints on field structure. With the advent of large 
optical telescopes, it has recently become possible to carry out such studies for a few stars, although at present the data have been restricted to circular spectropolarimetry which is a major limitation.

All studies assume that the magnetic field in the atmosphere is force-free and derivable from solutions of Laplace's equation (see however $\$ 3.1 .1$. Two approaches have been adopted. The first assumes a truncated multipolar expansion using spherical harmonics that include only the lowest harmonics (usually $l=2$ (dipole), $l=3$ (quadrupole), $l=4$ (octupole) with $m=0, . . l$ ). The series is arbitrarily truncated for computational efficacy which leads to restrictions on the type of field structures that can be modelled. The second approach, for which there is also no obvious physical justification, assumes that the field structure can be described by a combination of off-centred dipole, quadrupole and octupole zonal $(m=0)$ components, with arbitrary tilt angles and offsets for each of the components (see Euchner et al., 2002).

Detailed studies have been carried out for HE 1045+0908 and PG 1015+014 (Euchner et al., 2005, 2006). The best fit to the field structure of HE 1045+0908 is shown in Fig.9 (Euchner et al., 2005). For this star, the Zeeman tomographic analysis has revealed a field structure which is dominated by a quadrupole, but which also contains additional dipole and octupole contributions. The field structure in PG 1015+014 appears even more complex. Model fits using multipolar expansions with $l=1,2,3,4$ including tesseral components $(m \neq 0)$ give reasonable agreement with observations but also indicate that more terms are needed. The quality of the model fits appears to depend generally on which class of model is adopted, highlighting the fact that the inverse problem is not as yet adequately constrained. Stronger constraints will require improvements in dealing with the inverse problem, and the use of all four Stokes parameters.

\subsection{Convective mixing and accretion}

The hydrogen that is seen in the atmospheres of non-magnetic WDs could either be accreted, or be remnant from the time of formation. Sub-photospheric convection could mix in the outer $\mathrm{H}$ rich layers in certain ranges of effec- 


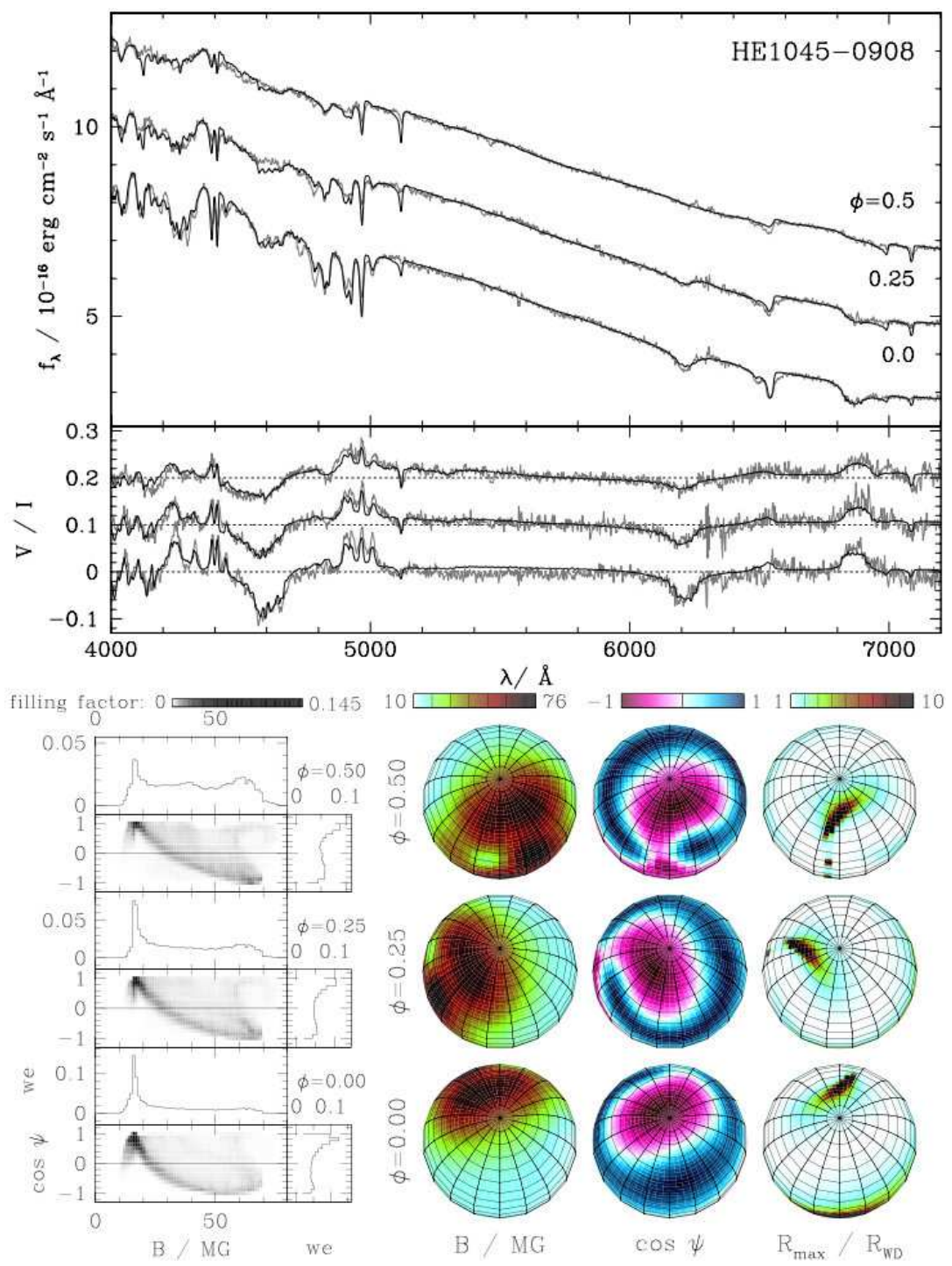

Figure 9: Zeeman tomographic analysis of the field structure of HE 1045+0908 (from Euchner et al., 2005) using an off-centred, non-aligned combination of dipole, quadrupole, and octupole. Top: Flux and polarisation data overlapped with the best fit model. Bottom left: $B-\psi$ diagram, where $\psi$ is the angle between the local field direction and the line of sight. Bottom right: absolute value of the surface magnetic field, cosine of $\psi$, and the maximum radial distance reached by field lines in units of the WD radius (see Euchner et al., 2005, for details). 
tive temperature, provided they are sufficiently thin, and result in a transformation of the spectral appearance from DA to DB as first suggested by Strittmatter and Wickramasinghe (1971). The surface atmospheric composition could also be altered by interstellar accretion or circumstellar accretion from a debris disc, and modified by gravitational diffusion, and the current evidence is that all these processes (accretion-diffusion-convective mixing) are in operation (see, e.g., Vauclair et al., 1979; Wesemael and Truran, 1982).

Magnetic fields can affect each of these processes to different extents. They may inhibit interstellar accretion or modify the accretion pattern by shielding the star from incoming plasma. The ratio $\zeta$ of ram pressure to magnetic pressure for a non-rotating star with a dipolar field moving with a velocity $v$ through an ambient medium of density $n_{\infty}$ can be estimated to be (e.g., Strittmatter and Wickramasinghe, 1971):

$$
\zeta \sim\left(\frac{n_{\infty}}{\mathrm{cm}^{-3}}\right)\left(\frac{M}{M_{\odot}}\right)^{6}\left(\frac{4 \times 10^{7} \mathrm{G}}{B}\right)^{2}\left(\frac{10^{9} \mathrm{~cm}}{R}\right)^{6}\left(\frac{50 \mathrm{~km} \mathrm{~s}^{-1}}{v}\right)^{2} .
$$

In the strong field case $(\zeta \leq 1)$ we expect an inhibition of accretion, while in the weak field case $(\zeta \geq 1)$ we may expect preferential accretion of ionised material on to the polar regions of the star. Given the strong dependence of $\zeta$ on $B$ we may expect patchy $\mathrm{H} / \mathrm{He}$ surface compositions in an otherwise $\mathrm{He}$ rich atmosphere due to differential effects on accretion in stars with fields of $\sim 1-4 \times 10^{7} \mathrm{G}$.

It is possible that a MWD with a field that is not strong enough to inhibit accretion $(\zeta \geq 1)$ if it is non-rotating, may inhibit accretion by the propeller mechanism if it does rotate. This mechanism could be important even in MWDs with modest magnetic fields $\left(\sim 5 \times 10^{7} \mathrm{G}\right)$ and rotation velocities $\left(\gtrsim 20 \mathrm{~km} \mathrm{~s}^{-1}\right)$.

A magnetic field may have a more direct effect on the atmospheric composition by inhibiting mixing of the outer layers of a WD with a convective envelope. This process may lead to the development of patchy surface chemical compositions even in weaker field stars. The work of Valyavin et al. (2014) indicates that magnetic fields may quell atmospheric convection and cause the formation of dark spots. At the high end of the field distribution convection can 
be suppressed altogether over the entire stellar surface thus altering the cooling evolution by making MWDs appear younger than they are. Using 3D radiation magneto-hydrodynamic simulations, Tremblay et al. (2015) confirmed that convection is suppressed by magnetic fields, however they also show that the cooling of the WD is not affected until the convective zone couples with the degenerate core, which occurs at effective temperatures below $6000 \mathrm{~K}$. Gentile Fusillo et al. (2018) provided evidence that magnetic fields suppress convection by modelling the ultraviolet and optical spectra of the MWD WD 2105-820, which has an effective temperature of $10000 \mathrm{~K}$ and should have a convective atmosphere. In their analysis, they showed that the ultraviolet and optical spectra resulted in consistent atmospheric parameters if the spectra were fitted with non-convective models. On the other hand, ultraviolet and optical spectra of non-magnetic WDs of similar temperature, had consistent atmospheric parameters when convective models were used. Given the above considerations, one may expect differences in the atmospheric properties of non-magnetic and MWDs, and there is some evidence that this may indeed be the case.

Feige 7 is rare among MWDs in showing both $\mathrm{H}$ and He lines in its spectrum. The spectrum varies with a rotation period of $2.19 \mathrm{hrs}$ and can be interpreted as evidence for a patchy surface chemical composition with some regions being more hydrogen rich than others (Achilleos et al., 1992). The star has an effective temperature of $\sim 21,000 \mathrm{~K}$ in the regime where convective mixing is expected to take place.

In addition to spectral line variations, rotating MWDs are expected to also exhibit variations in continuum flux and polarisation as the underlying magnetic field is viewed at different magnetic phases. Such a behaviour is typified again by Feige 7 (see Wickramasinghe and Ferrario, 2000) that exhibits variations at a level of $4 \%$ in $\mathrm{V}$ which can be explained in terms of a patchy surface composition and the field dependence of continuum opacities (magnetic dichroism).

Flux variations at the $0.2 \%$ level has been detected in the low-field MWD WD 1953-011. This star has an effective temperature of $\sim 7900 \mathrm{~K}$ and a convective atmosphere (Maxted et al., 2000; Brinkworth et al., 2005). The spectral 
line variations have been interpreted in terms of a two component model consisting of an underlying dipolar field distribution with $B_{d}=7 \times 10^{4} \mathrm{G}$ and a spot with a field of $5 \times 10^{5} \mathrm{G}$ covering $\sim 10 \%$ of the stellar surface (Maxted et al., 2000). The magnetic field strength appears too weak to explain the photometric changes purely in terms of magnetic dichroism (see Wickramasinghe and Ferrario, 2000). Larger scale changes in opacity due to variations in the atmospheric $\mathrm{He} / \mathrm{H}$ ratio across the surface may be a more plausible explanation, although unlike with Feige 7, the star is too cool to show helium lines. Another possibility is that chemical inhomogeneities play a secondary role and that the magnetic field has a direct influence on the thermal structure of the atmosphere by inhibiting convective motions perpendicular to field lines. The changes in atmospheric structure may then lead to small scale variations in the optical flux over the stellar surface (Brinkworth et al., 2005).

\subsection{Planets around magnetic WDs}

In this section we shall focus specifically on the detection of planets using methods that are contingent on the presence of a magnetic field in the WD.

GD 356 is so-far the only WD to show the Balmer series in pure emission. The emission lines are split into Zeeman triplets and the lack of significant field broadening suggests that the lines originate from a region of the stellar surface with a nearly uniform field of $13 \pm 2 \mathrm{MG}$ (Ferrario et al., 1997b). The appearance of emission rather than absorption lines can be explained if there is a temperature inversion in the outer layers that begins at significant optical depth. Nearly sinusoidal low amplitude $(0.2 \%)$ photometric $\mathrm{V}$ band variability has been detected in this star, yielding a rotation period of $\sim 115 \mathrm{~min}$ (Brinkworth et al., 2004).

The origin of the chromospheric type temperature inversion in GD 356 has received much attention. One possibility is that the atmosphere is heated by X-ray emission from a corona, but searches for such emission have led to negative results with an upper limit of $6 \times 10^{25} \mathrm{erg} \mathrm{s}^{-1}$ to the X-ray luminosity being placed from Chandra observations (Weisskopf et al., 2007), an order of 
magnitude lower than what is required to explain the luminosity in Balmer line emission (Ferrario et al., 1997b). The absence of detectable X-ray emission also argues against heating caused by accretion from a brown dwarf companion or the interstellar medium. Further studies by Wickramasinghe et al. (2010) using Spitzer Infrared Array Camera observations have confirmed this by placing a strong upper limit of $12 \mathrm{M}_{\mathrm{J}}$ (where $\mathrm{M}_{\mathrm{J}}$ is the mass of Jupiter) on the mass of a possible companion. In view of this result, models invoking accretion from a companion star can be dismissed.

Another intriguing possibility is that GD 356 has an Earth-type planetary companion that causes the heating of the atmosphere of the WD by the unipolar inductor mechanism (see Li et al., 1998, who first suggested this possibility). On this model, the resistive dissipation of electrical currents that flow between the conductive iron core of an Earth-like planetary companion (stripped of its mantle during the red giant evolution of its star) and the MWD results in the heating of the upper atmosphere of the MWD. The model is similar to that proposed for the Io-Jupiter system and predicts the existence of heated regions near the footpoints of closed field lines which is generally consistent with the constraints provided by the Zeeman modelling. A schematic diagram illustrating this model is shown in Fig.10 (Li et al., 1998).

The fate of planetary systems around main sequence stars that evolve into WDs is not well understood. Rocky planets that are dragged in during the redgiant phase of evolution of the parent star may be either fully encompassed by the star or their cores may survive in close orbit around the WD. According to Willes and $\mathrm{Wu}(2005), \sim 0.1 \%$ of main-sequence planetary systems may evolve into MWD - Earth type planet systems. These planets may be detectable via the unipolar inductor mechanism before they are tidally disrupted. A subset of these systems may emit radio emission by the electron-cyclotron maser mechanism at levels that may be detectable in future radio surveys in the $\sim 50-500 \mathrm{GHz}$ band (Willes and Wu, 2005).

It may be possible to detect brown dwarfs and gaseous giant planets around MWDs through the detection of cyclotron emission from the MWD. For in- 


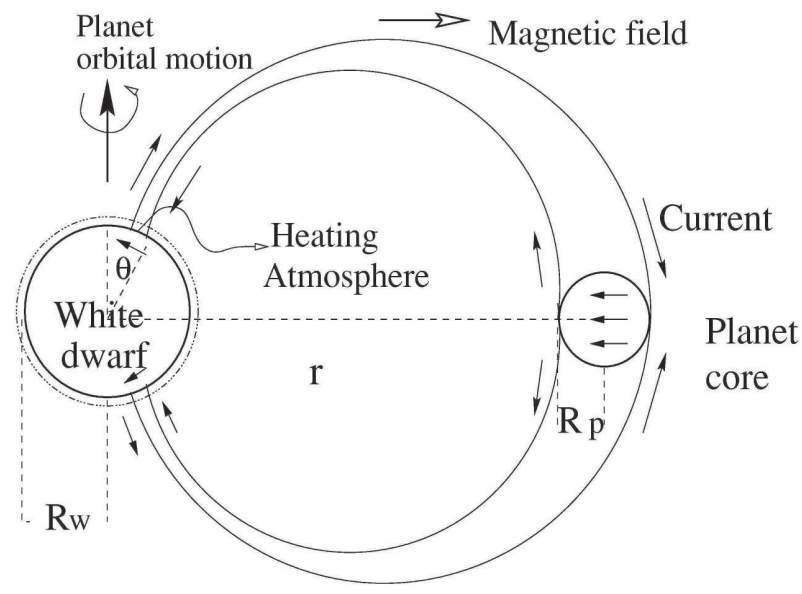

Figure 10: The highly conductive iron core of a planet acts like a battery by generating electric currents as it moves in the magnetosphere of the MWD. The electric circuit that is setup causes the heating of the MWDs atmosphere (the circuit's resistor) near its magnetic poles causing a temperature inversion and the formation of emission lines (see Li et al., 1998, for details). 
stance, the system SDSS J1212+0136 (Schmidt et al., 2005a) has an orbital period of 88.4 min and consists of a L7 brown dwarf (Debes et al., 2006) orbiting a MWD with a field of $\sim 7 \mathrm{MG}$. The cyclotron emission from the accretion shock on the surface of the MWD is powered by wind accretion (as opposed to Roche lobe overflow) from its brown-dwarf companion (Schmidt et al., 2005a). The strength of these cyclotron lines, and hence their detectability, depends on the specific accretion rate, but it is not clear whether Jupiter-like giant planets have winds of sufficient strength to produce measurable cyclotron emission.

There have been reports of the detection of planets in a number of CVs, among which the short-period DP Leo (a MCV that belongs to the polar subclass) by Beuermann et al. (2011) who found convincing evidence that the third body orbiting this system is a giant planet in an elliptical orbit. This suggests that the occurrence of planets or planetary systems in post-CE binaries may not be a rare incidence. However, it is unclear whether these planets are first generation, that is, they were born together with their star in a proto-planetary disc, or second generation, that is, born from the material that was ejected during the AGB super-wind phase and/or common envelope evolution. Some $25 \%$ of WDs show the presence of metals in their spectra caused by ongoing accretion of planetary debris (see $\$ 4.5$ ). This and the detection of gaseous debris discs around many WDs strengthen the hypothesis that planets exist or did exist around a large fraction of WDs.

\section{Population properties of magnetic WDs}

\subsection{The incidence of magnetism}

MWDs with fields in the range $10^{6}-10^{9} \mathrm{G}$ are readily recognised even at low spectral resolution $(\sim 10 \AA)$ and thus many MWDs have been discovered in surveys such as the Sloan Digital Sky Survey (SDSS), the Hamburg/ESO survey and the Edinburgh-Cape survey. Interestingly, the early work of Putney (1997) revealed that the incidence of magnetism among WDs with no detectable absorption features (the DC WDs) is close to $22 \%$, which is much higher than 
among the general population of WDs. Further studies conducted over the following years revealed that a significant fraction of the magnetic DC discovered in this survey were in fact cool DAP white dwarfs. A similar survey conducted on the current much larger sample of DC WDs may lead to the discovery of a considerable number of new MWDs.

Higher resolution spectroscopic studies capable of measuring even lower fields have usually been limited to a few bright WDs. The high resolution $(\sim 0.1 \AA)$ ESO Supernova Ia Progenitor Survey (SPY) of more than 1,000 WDs presented an opportunity for the study of magnetism in the range $\sim 10^{5}-10^{6} \mathrm{G}$. However, Koester et al. (2009)) reported that SPY only detected nine MWDs with fields below $10^{6} \mathrm{G}$ (five new and four already described in the literature), a result that seems to be generally consistent with spectropolarimetric surveys also sensitive to this field range (see below).

Despite the large increase in the number of newly discovered MWDs, the question of the incidence of magnetism among WDs remains unresolved. Early studies based on magnitude limited surveys, such as the PG survey, indicated that $\sim 2 \%$ of WDs are magnetic. However, such surveys were biased against the discovery of massive and hence fainter WDs. A re-analysis of the data of the PG survey taking into account the different volumes that are sampled by different mass WDs resulted in an upward revision of the percentage of MWDs to at least $\sim 10 \%$ (Liebert et al., 2003). This revision rests on the observation that MWDs with fields $\geq 10^{6} \mathrm{G}$ tend on average to have higher masses than their non-magnetic counterparts (see $\S 4.2$ ).

A complementary approach has been to isolate and study the properties of nearly complete volume limited samples of nearby WDs using field determinations from spectroscopy and spectropolarimetry. For the 13 pc sample, it has been estimated that $21 \pm 8 \%$ of all WDs have magnetic fields greater than $\sim 3 \mathrm{kG}$, while for the $20 \mathrm{pc}$ sample, an incidence of $13 \pm 4 \%$ has been estimated (Kawka et al., 2007). In this statistical analysis, however, it is suggested that the 20 pc sample is incomplete at the kilo-Gauss level. The more recent work of Holberg et al. (2016) corroborated these earlier findings by showing that the in- 
ner 20 pc volume contains $12 \%$ MWDs with a space density of $\sim 0.6 \times 10^{-3} \mathrm{pc}^{-3}$.

The search for low-field MWDs $\left(B<10^{6} \mathrm{G}\right)$ is based almost exclusively on spectropolarimetric surveys, except for the few bright WDs for which high resolution spectra are available. A survey of 169 mainly northern WDs (Schmidt et al., 1995, and references therein) led to the discovery of the first two low field WDs: LP 907-037, with a longitudinal field $B_{l}=8.5 \times 10^{4} \mathrm{G}$, and G 217-037, with $-100 \pm 15 \leq B_{l} \leq+9 \pm 12 \mathrm{kG}$. An extension of this survey to include 60 southern hemisphere WDs (Schmidt et al., 2001) led to the discovery of another possible low-field MWD with a longitudinal field of $6.1 \pm 2.2 \mathrm{kG}$. Other spectropolarimetric studies with sensitivities capable of detecting kilo-Gauss fields resulted in the discovery of many more low-field MWDs. However, the sample of low-field MWDs, based on detections of polarisation across spectral lines, is still not large enough to establish the real incidence of very weak fields in WDs (Landstreet et al., 2012; Bagnulo and Landstreet, 2018).

The current evidence is that the magnetic field distribution of WDs has a well defined high field component $\left(10^{6}-10^{9} \mathrm{G}\right.$, forming the class of high-field MWDs) whose incidence is about $3 \%$ in magnitude-limited surveys, and a low field component ( $<10^{6} \mathrm{G}$, forming the class of low-field MWDs) whose incidence and properties are not as well established at the present time. The magnetic field distribution of all known MWDs is shown in Figure 11.

\subsection{The Mass distribution}

The problem concerning the determination of surface gravities and masses from the line spectra of MWDs has always been intractable except in low field stars, i.e., $B \leq$ a few $\times 10^{6} \mathrm{G}$. In this regime it is assumed that the magnetic field does not affect the atmospheric structure so that field broadening is small and existing Stark broadening theories (at zero magnetic field) can be used to model the line wings (e.g. Ferrario et al., 1998).

In principle, it should also be possible to use stationary components that are insensitive to field structure (see $\S 3.2$ ) to estimate gravities from line profiles. However, this strategy must await a full theory of Stark broadening in the 


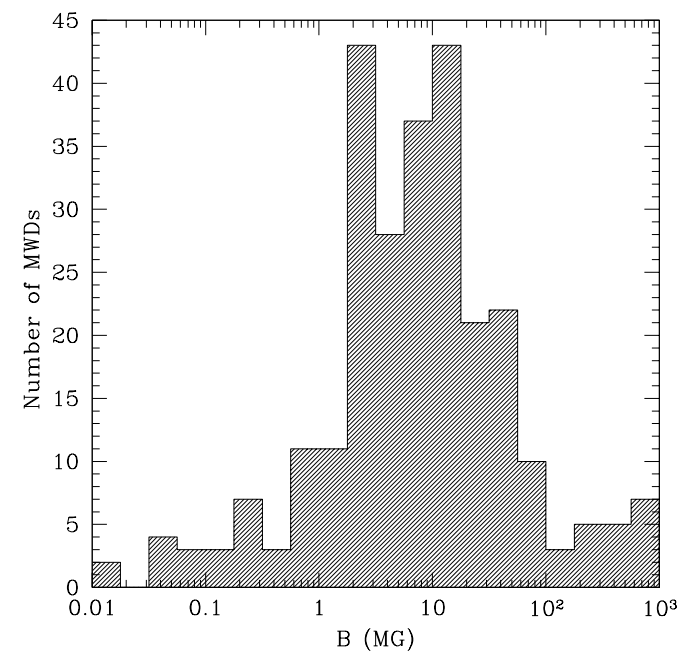

Figure 11: The magnetic field distribution of all isolated MWDs (this work).

presence of crossed electric and magnetic fields, and the construction of more realistic model atmospheric structures that allow for the effects of the magnetic field. Although this is an important and unsolved problem pertaining to the physics of MWDs, their masses can now be found thanks to the parallax measurements provided by the Gaia mission. It is encouraging to see that the mass determinations for low field MWDs that were made through spectroscopic studies compare well with those provided by the parallax measurements of GAIA. Furthermore, the masses of MWDs that are determined through parallax will help us constrain Stark broadening theories (and atmospheric models) that allow for the presence of strong fields.

We show in Fig.12 the mass distribution of hydrogen-rich MWDs that have SDSS (DR12) photometry and Gaia trigonometric parallax. The data in Figure 12 confirm that the MWDs as a group have a higher than average mean mass as first noted by Liebert (1988). The discovery by Kilic et al. (2018) that nonmagnetic WDs show two very distinct populations, one peaking near $0.6 \mathrm{M}_{\odot}$ and the other near $0.8 \mathrm{M}_{\odot}$, is very surprising (see Fig.13). The explanation of 

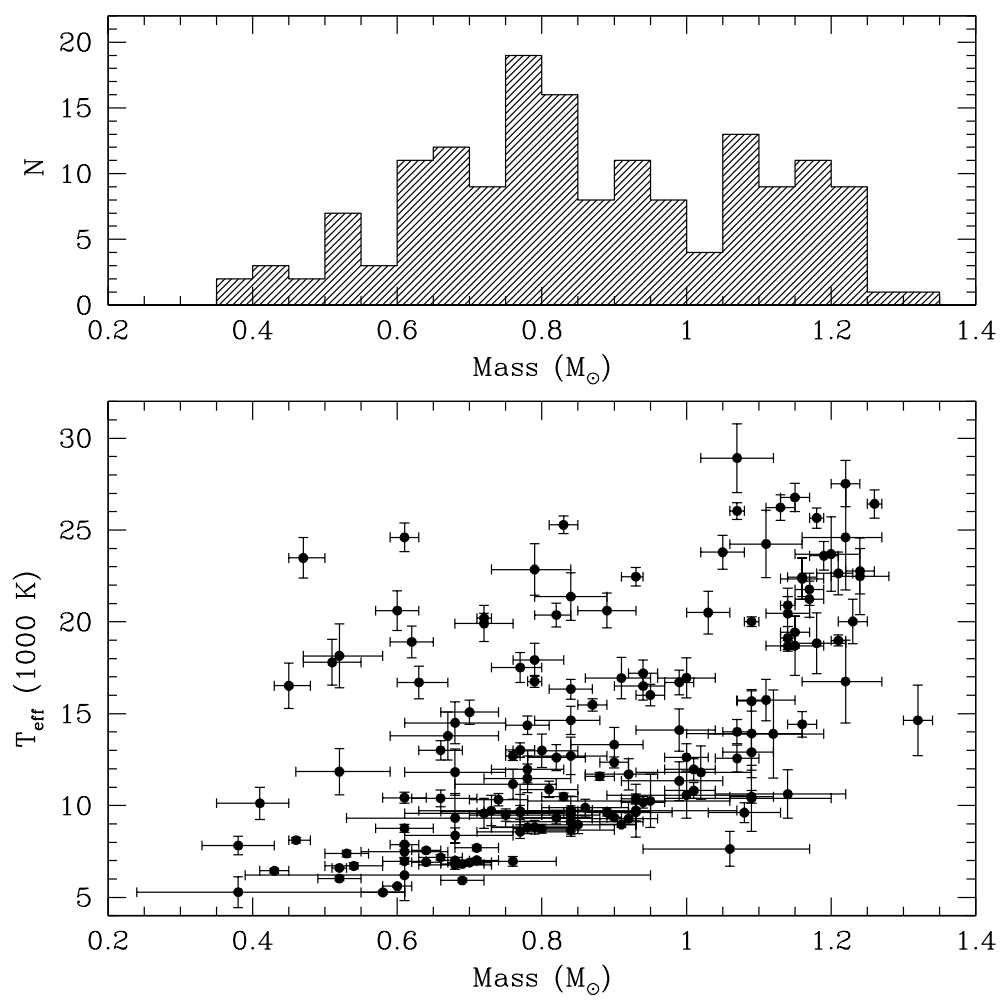

Figure 12: Top panel: histogram of the mass distribution of hydrogen-rich MWDs that have SDSS photometry and Gaia parallaxes. Bottom panel: Effective temperature of these MWDs against masses (this work). 
Kilic et al. (2018) is that the more massive population is the likely result of stellar merging events. However, a word of caution is in order, since Bergeron et al. (2019) noted that some of these high mass WDs could be $\sim 0.6 \mathrm{M}_{\odot}$ WDs with a mixed $\mathrm{H} / \mathrm{He}$ atmosphere. Thus, the magnitude of this second high mass peak can only be established by conducting further observational studies to determine the atmospheric composition of this group of stars. Nonetheless, the existence of a large population of massive merged WDs is consistent with the studies of Holberg et al. (2016) of the WDs in the $13 \mathrm{pc}$ volume-limited sample. They find that the incidence of WDs in systems containing two or more stars is only 26\%. As first noted by Ferrario (2012), who studied an earlier volume-limited sample, this finding is at odds with observations showing that at least $50 \%$ of intermediate mass main-sequence stars (the progenitors of the WDs) reside in multiple systems. The explanation provided by Ferrario (2012) was that the missing WDs may be concealed either in double degenerate systems or in Sirius-like systems. However, it now appears more likely that a large fraction of binaries merge during the post-main sequence phase. This topic will be covered extensively in $\S 6$.

\subsection{Rotational Periods}

The spectroscopic determination of the rotational periods of WDs is very difficult because of their extremely pressure-broadened lines. However, the $\mathrm{H}_{\alpha}$ absorption lines exhibit a very weak and narrow non-NLTE core that has allowed the studies of the rotational properties of hot $\left(T_{\text {eff }} \gtrsim 14,000 \mathrm{~K}\right) \mathrm{DA}$ WDs. Such studies have yielded null detections in the vast majority of WDs resulting in upper limits $\mathrm{v} \sin \mathrm{i} \leq 20 \mathrm{~km} \mathrm{~s}^{-1}$ being placed on the projected rotational velocities (Karl et al., 2005) which corresponds to lower limits of $P_{\text {rot }} \geq 1 \mathrm{hr}$ to the rotational periods.

Rotational periods can also be determined from the rotational splitting of the oscillation modes in pulsating WDs. Some well studied systems (see, e.g., Winget et al., 1991; O'Brien et al., 1996) have yielded rotation periods in the range $1.17-1.60 \mathrm{~d}$ corresponding to velocities of a few $\mathrm{km} \mathrm{s}^{-1}$. This may suggest 


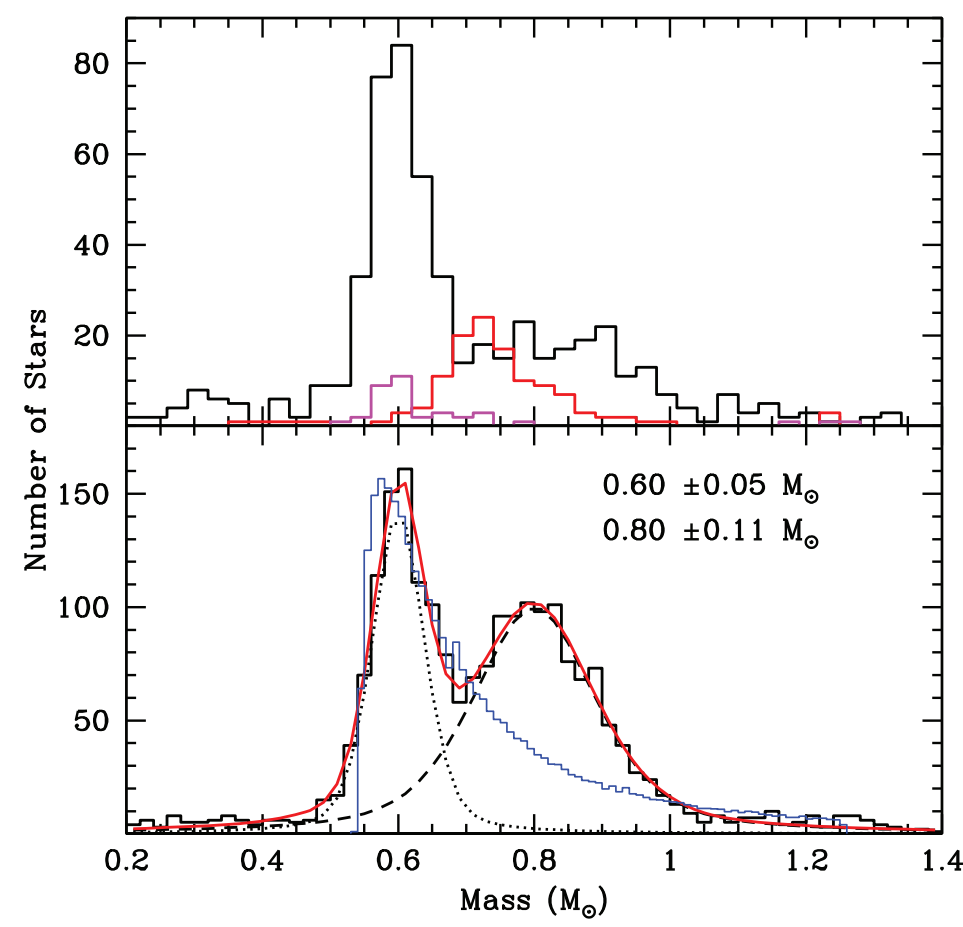

Figure 13: Top panel: mass distributions of SDSS DA, DB and DC non-magnetic WDs, depicted in black, magenta, and red respectively (see Kilic et al., 2018, for full details). Bottom panel: mass distribution of the $100 \mathrm{pc}$ non-magnetic WDs with $T_{\text {eff }} \geq 6,000 \mathrm{~K}$. The presence of two distinct populations peaking near $0.6 \mathrm{M}_{\odot}$ and $0.8 \mathrm{M}_{\odot}$ is clearly evident (see Kilic et al., 2018, for full details). 
a few days as the typical rotational period for non-magnetic WDs although significantly longer periods are much more difficult to establish without long term observations. The more recent studies of Hermes et al. (2017) of 27 pulsating hydrogen-rich WDs, the DAVs or ZZ Ceti stars, observed by the Kepler mission (Basri et al., 2005) provided constraints on the spin period of 20 of them, doubling the number of WDs whose spin periods are derived through asteroseismology. Follow-up spectroscopic observations, also conducted by Hermes et al. (2017), provided WD's rotation as a function of mass. They find that WDs in the mass range $0.51-0.73 \mathrm{M}_{\odot}$, which are the progenies of main sequence stars in the mass range $1.7-3.0 \mathrm{M}_{\odot}$, have an average spin of $35 \pm 28 \mathrm{hr}$ while the three most massive WDs in their sample seem to rotate much faster $(4.0 \pm 3.5 \mathrm{hr})$. The measured values and upper limits are significantly smaller than the breakup speed. For instance, a $3 \mathrm{M}_{\odot}$ main-sequence star that rotates uniformly with an equatorial velocity of $\sim 200 \mathrm{kms}^{-1}$ would be expected to generate a WD with a rotation period of about $\sim 20 \mathrm{~s}$ which is not verified by observations. This is evidence of efficient transfer of angular momentum from the stellar core to the envelope during pre-WD evolution. Thus, core and envelope must be strongly coupled during the evolution with powerful stellar winds, particularly during the AGB evolution, carrying away most of the star's angular momentum (Ferrario and Wickramasinghe, 2005). The asteroseismology studies of about 300 red giant stars with data from the Kepler mission have verified this hypothesis showing that degenerate cores already rotate more slowly than expected when they are at the end of the red giant branch evolution (Mosser et al., 2012).

In the case of the high-field MWDs, photometry and polarimetry can be used to determine or place more stringent limits on rotational velocities. Early studies of a few high-field MWDs showed no variations in polarisation over periods of tens of years. This was taken as evidence that they had periods $\geq 100$ yrs. The unlikely possibility that these stars may in fact have rotation periods that are significantly shorter than the typical integration times $(\sim$ hrs $)$ used in early studies was subsequently ruled out for Grw $+70^{\circ} 8247$ and LP 790-29 (Jordan and Friedrich, 2002) by fast time resolution polarimetric studies. By 
using the changes in the polarisation position angle, which gives the rotation of the magnetic axis in the plane of the sky, Bagnulo and Landstreet (2019) confirmed that the rotation period of $\mathrm{Grw}+70^{\circ} 8247$ is likely to be in the range $100-1,000$ years. Polarimetric variations have also been detected on a timescale of $\geq 10$ yrs in the high-field MWDs GD 229 and G 240-72 with implied rotational periods of $\sim 100 \mathrm{yrs}$ (Berdyugin and Piirola, 1999). The subsequent work of Brinkworth et al. (2013) confirmed the absence of short term variability in G 240-72. Thus, it appears that there is a sub-class of high-field MWDs that is very slowly rotating which may be evidence that magnetic fields play an additional important role in the removal of angular momentum. The photometric studies of Brinkworth et al. (2013) of 21 high-field MWDs revealed that $67 \%$ are variable and $24 \%$ have measurable rotation periods. They find no correlation between rotation and other WD parameters, such as mass, temperature, magnetic field, or age. Fig.14 shows the magnetic field versus rotation period of all currently known MWDs. Whilst we also find that there is no correlation between field strength and period, we also note that only strongly magnetic MWDs exhibit very long rotational periods.

There is also a significant class of rapidly rotating MWDs consisting of stars such as RE J0317-853 ( $P_{\text {rot }}=12 \mathrm{~min}$ ) (Barstow et al., 1995; Ferrario et al., 1997a). RE J0317-853 is believed from other considerations to be the result of a merger (Ferrario et al., 1997a; Vennes et al., 2003). We note that MWDs that are spun up in interacting binaries through the action of accretion torques could have typical rotation periods of hours. Some of the isolated MWDs in the rapidly rotating group could therefore be mergers or the end products of the binary evolution of MCVs with very low-mass companions.

\subsection{The magnetic $D Q$ white dwarfs}

One of the most remarkable discovery in recent years is that of DQ WDs that are hot $(\sim 15000-24000 \mathrm{~K})$ and whose atmospheres are carbon dominated $(-2 \lesssim \log [\mathrm{C} / \mathrm{He}] \lesssim 2)$. These objects show no $\mathrm{H}$ or HeI lines in their optical spectra. Instead, they exhibit absorption lines of C II at 4267, 4300, 


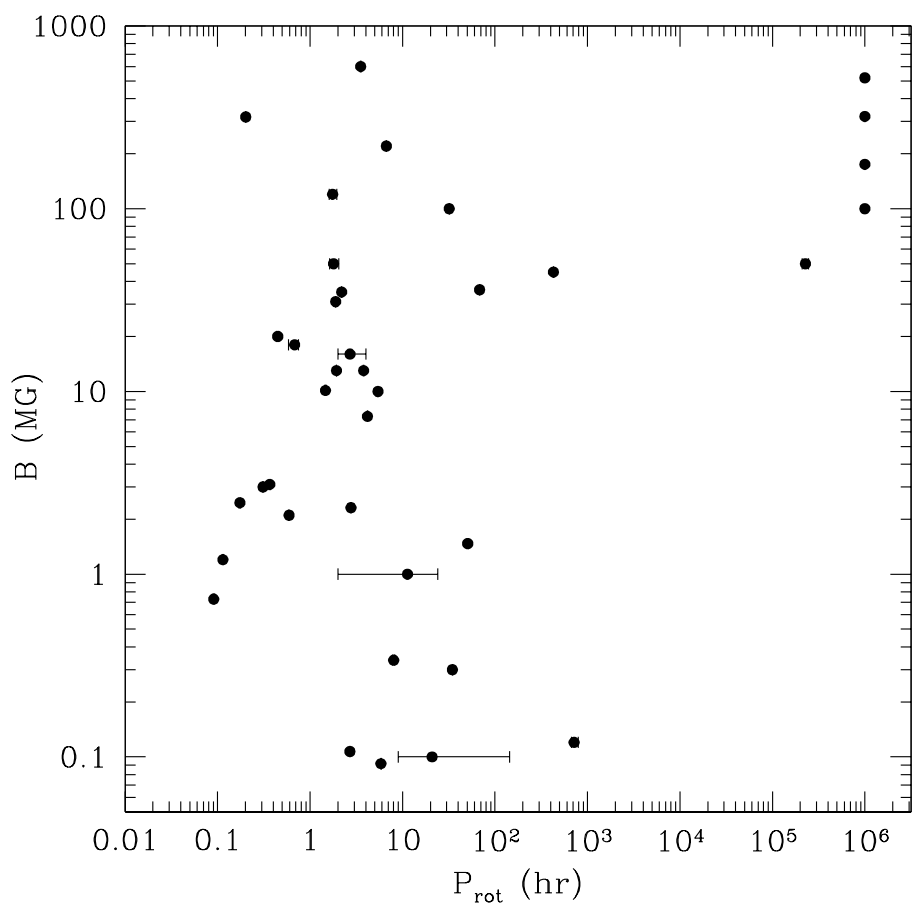

Figure 14: The dependence of rotational period on magnetic field. Stars with estimated rotational periods of greater than $100 \mathrm{yrs}$ are plotted with a period of $100 \mathrm{yrs}$. 


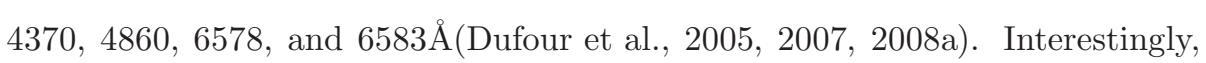
there are no DQ WDs with temperatures over 24,000 K. Furthermore, approximately $70 \%$ of them are magnetic (Coutu et al., 2019; Dufour et al., 2013; Dunlap and Clemens, 2015, and Kawka et al. (2020), in preparation). Werner (2012) have proposed that objects with CO dominated atmospheres (such as H 1504+65 Nousek et al., 1986, which is extremely hot $(\sim 200,000 \mathrm{~K})$, massive $\left(0.85 \pm 0.15 \mathrm{M}_{\odot}\right)$, and shows no $\mathrm{H}$ or He lines in its spectrum), could be the progenitors of the hot DQs. Coutu et al. (2019) suggested that the massive $\left(M \geq 0.8 M_{\odot}\right)$ warm DQs $\left(10000 \leq T_{\text {eff }} \leq 16000 \mathrm{~K}\right)$ could be the descendants of the hot DQs. Most of these warm DQs are not known to be magnetic, but this could simply be due to the poor signal-to-noise of the spectra used in their analysis. High-resolution spectroscopy or spectropolarimetry with high signalto-noise is necessary to determine the incidence of magnetism among warm DQs.

A possible explanation for the origin of these carbon-dominated objects is that their AGB precursors could have undergone a final thermal pulse that resulted in the ejection of most, if not all, their helium envelopes. A reasoning along the same lines was proposed to explain the existence of hydrogen-poor WDs (e.g., Werner and Herwig, 2006, and references therein). However, this does not explain why so many hot DQs are also massive and magnetic. Two possibilities have been investigated: (i) they could be the progenies of the most massive main-sequence stars that can evolve into WDs $\left(5 \lesssim M / \mathrm{M}_{\odot} \lesssim 8\right)$, or (ii) they could be the result of stellar merging events, as proposed by Wickramasinghe (2014b) and Briggs et al. (2015) to explain the origin of fields in all high-fields MWDs (see $\S[6)$. At present it is unclear how stellar merging occurs and whether it could lead to the ejection of most of the He layers of the merged object. However, because the kinematic properties of the hot DQ WDs are those of an older stellar population (Dunlap and Clemens, 2015), the stellar merging hypothesis seems to be by far the most likely cause for their origin (Coutu et al., 2019). This merging scenario is strongly supported by the recent study of (Kawka et al., 2020) of the ultra-massive warm DQ WD LP93-21 whose physical properties and 
highly retrograde halo kinematics can only be explained if this object is the outcome of an ancient double degenerate merger. We show in Fig.15 the carbon abundance as a function of the effective temperature for all known DQ WDs (hot and warm DQs plus also the cool DQ WDs described in section 2.1.4). The

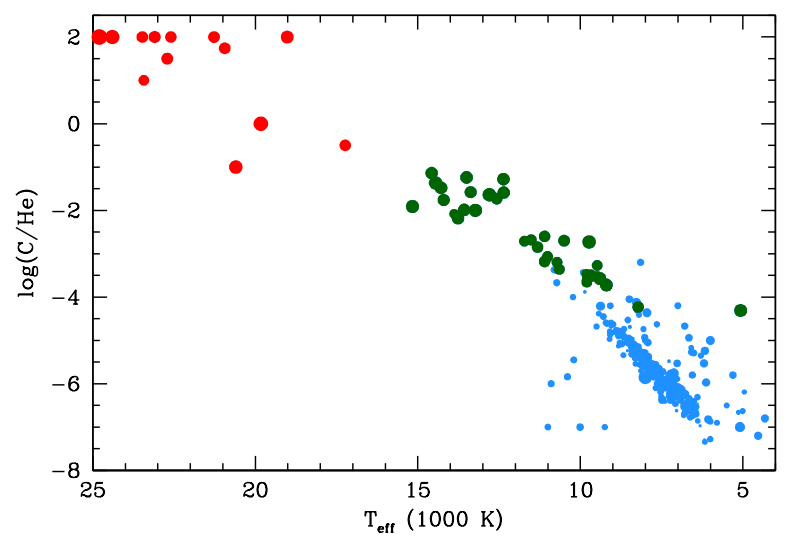

Figure 15: Carbon abundances as a function of the effective temperature for known DQ stars. The point size is a function of the mass of the WD. Normal, low-mass DQs are depicted in blue. The higher mass warm DQs are in green and the hot and massive DQs are in red (Kawka et al., 2020a, in preparation).

temperature range of hot DQ WDs is such that some of them are expected to be pulsating (Fontaine et al., 2008). Hydrogen-rich WDs pulsate at temperatures $\left(T_{\text {eff }} \approx 12000 \mathrm{~K}\right)$ where $\mathrm{H}$ is partially ionized. Similarly, helium-rich WDs pulsate where helium is partially ionized at $T_{\text {eff }} \approx 25000 \mathrm{~K}$. Fontaine et al. (2008) showed that the effective temperature of the small number of known hot DQs fall in the temperature range where carbon is partially ionized, and therefore should pulsate in non-radial g-modes similarly to WDs with hydrogen and helium dominated atmospheres. These pulsations can then be used to probe the interior structure of the WD. A search for pulsations in this class of objects led to the discovery by Montgomery et al. (2008) of the first pulsating, carbondominated WD, SDSS J142625.71+575218.3, which exhibits a period of $417 \mathrm{~s}$. Further observations of this intriguing WD conducted by Dufour et al. (2008b) revealed that it is not only pulsating, but also possesses a $1.2 \mathrm{MG}$ field, as the 
spectra of Dufour et al. (2008b) depicted in Fig.16 show.

Following the discovery of photometric variations in SDSS J142625.71+575218.3, several more hot DQ WDs have been found to be variable. Although this variability was originally attributed to pulsations, the lack of multi-mode variations, expected from pulsating WDs, and the discovery of a $2.1 \mathrm{~d}$ photometric modulation in the hot DQ WD SDSS J000555.9-100213.5 (Lawrie et al., 2013) led to the suggestion that the variations are not caused by pulsations but by rotation (Williams et al., 2016). This means that hot DQ WDs are generally characterised by rapid rotation which again supports the hypothesis that these objects originate from merging.

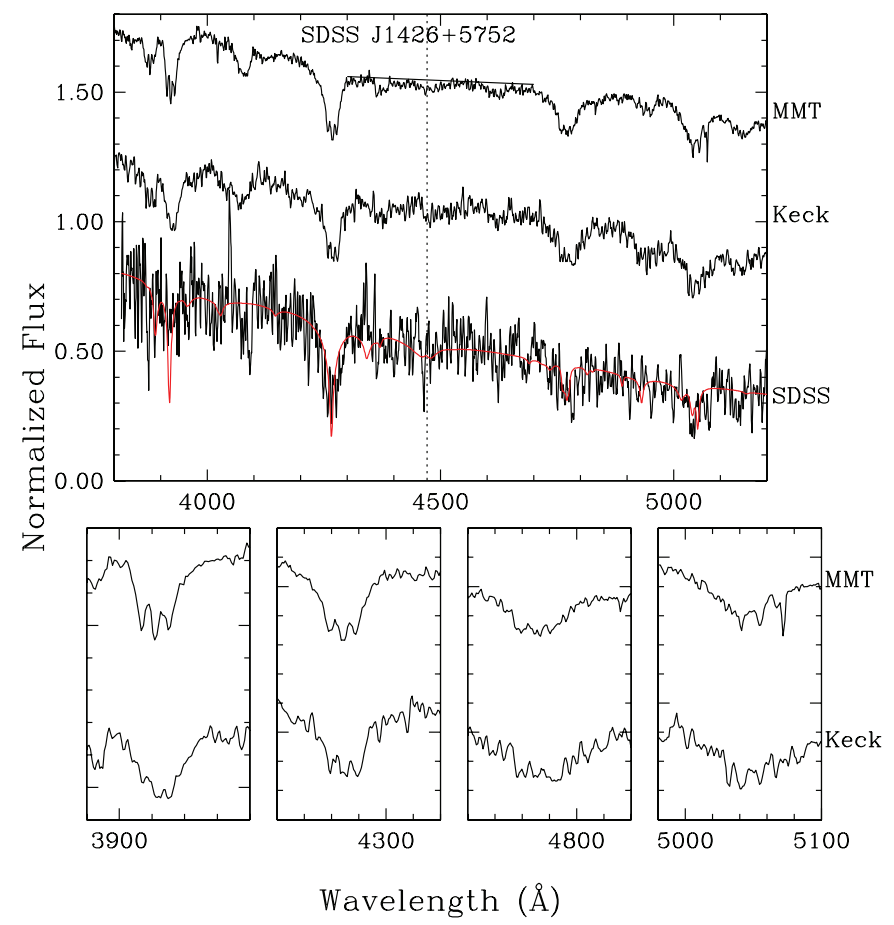

Figure 16: Top panel: Spectra of SDSS J142625.71+575218.3 with overlapped in red the best, non-magnetic, fit (see Dufour et al., 2008b, for full details). The dotted line indicates the position of the HeI 4471 line. Bottom panel: Carbon lines from the Keck and MMT spectra showing the presence of a 1.2 MG magnetic field (see Dufour et al., 2008b, for full details). 


\subsection{The magnetic $D A Z$ and $D Z$ white dwarfs}

As noted in $\S 2.1 .5$ about $25 \%$ of WDs show traces of metals in their spectra. For this metal pollution to exist it is necessary to assume ongoing accretion of planetary debris (Jura, 2003) because the diffusion time-scales for these elements to sink is too short. This idea is strengthened by the discovery of gaseous and dusty discs around WDs (Becklin et al., 2005; Jura et al., 2007; Farihi et al., 2008, 2009). A subset of metal polluted WDs exhibits the presence of magnetic fields (Reid et al., 2001; Kawka and Vennes, 2011; Farihi et al., 2011; Kawka and Vennes, 2014). The studies of Kawka and Vennes (2014) have shown that the incidence of magnetism is higher in cool $(<6,000 \mathrm{~K})$ and polluted hydrogen-rich WDs (denoted as DAZ) with fields measured to be in the range $\sim 70-500 \mathrm{kG}$ (Kawka et al., 2019). The incidence drops to essentially zero at temperatures above about 7,000 K. The studies of Kawka et al. (2019) of the current sample of DAZ WDs is summarised in Fig.17 which clearly shows that the incidence of magnetism is close to $50 \%$ at $T_{\text {eff }}<7,000 \mathrm{~K}$. The reason for this higher incidence of very weak fields at low temperatures is still unknown. Kawka et al. (2019) speculated that it could be caused by a gaseous planet plunging onto a WD. Such an event may instigate differential rotation over a large enough outer layer of the WD to generate the very weak fields detected in cool DAZ WDs. This mechanism was first suggested by Farihi et al. (2011) to explain G77-50. According to their picture, the orbits of outer planets and asteroids around the WD G77-50 could have been disrupted by a close encounter with another star. If, as estimated by Farihi et al. (2011), such an encounter has a $50 \%$ chance of happening every $0.5 \mathrm{Gyr}$, then older WDs would be more likely to have accreted a gaseous giant planet during their motion around the Galactic centre than their younger counterparts.

Similarly, Hollands et al. (2015) showed that cool, helium-rich DZ WDs exhibit a high incidence of magnetism, i.e., about $13 \%$ at $T_{\text {eff }} \leq 8000 \mathrm{~K}$ dropping rapidly at higher temperatures. Another peculiarity is that the strengths of magnetic fields in cool DZ WDs have a range from weak fields, comparable to the field strengths in cool DAZ WDs, to much stronger fields in excess of $10 \mathrm{MG}$ 
(Hollands et al., 2015, 2017). It is possible that this is entirely a selection effect because the metal lines in DZ WDs are much stronger than in DAZ WDs. Therefore at higher fields the weaker metal lines may be smeared out by magnetic field broadening (see 3.2). The metal lines also become weaker at higher temperatures.
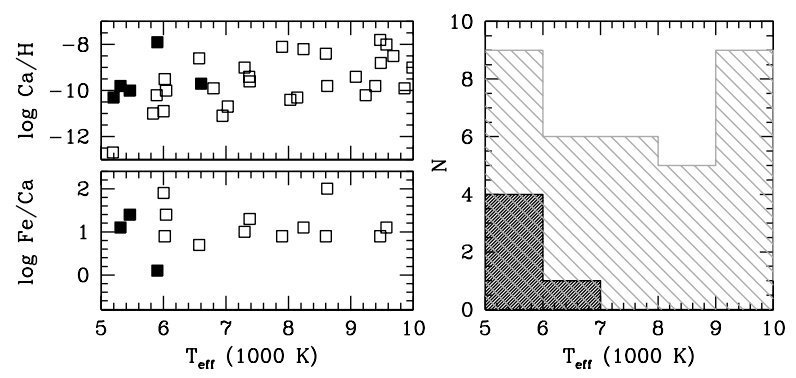

Figure 17: Left: The abundances of cool DAZ WDs. The full squares represent the MWDs while the open squares the non-magnetic WDs. Right: The effective temperature distribution of DAZ WDs. The magnetic DAZ WDs are shaded in dark grey (from Kawka et al., 2019).

\subsection{Chromospheres, coronae and contemporary dynamos in WDs?}

WDs develop convective envelopes as they cool, driven by the ionisation of HeI and HeII. It has been suggested that cool WDs with deep convective envelopes may generate fields of $\sim 100 \mathrm{kG}$ driven by an $\alpha-\omega$ dynamo (Thomas et al., 1995). Such fields may be expected to lead to the formation of hot coronae with X-ray luminosities of $10^{27}-10^{30} \mathrm{erg} \mathrm{s}^{-1}$ (Winget et al., 1994). However, searches for coronal X-ray emission from cool WDs have so far led to negative results (see Musielak et al., 2003, and references therein). It is possible that in the case of WDs, the wave flux energy is dissipated in a chromosphere rather than a corona (Musielak et al., 2005). However, chromospheric activity is also not a general characteristic of MWDs.

From an observational point of view, there is no strong evidence to support that the incidence of magnetism increases in cooler WDs (but see $\$ 4.7$ ) as may be expected if a contemporary dynamo were in operation nor have there 
been reports on reversals in polarity in any MWDs. Current observations are, however, limited to WDs with fields significantly larger than $10^{3} \mathrm{G}$.

\subsection{Complexity of field structure, field decay and field evolution}

The free Ohmic-decay time can be approximated by $t_{\mathrm{ohm}} \sim \frac{4 \pi \sigma L^{2}}{c^{2}}$ where $L$ is the magnetic field's variability scale-length and $\sigma$ is the electrical conductivity. If we take $L \sim R$ (WD's radius) and $\sigma$ consistent with what is expected in a WD's degenerate core, then $t_{\mathrm{ohm}} \sim 2-6 \times 10^{11} \mathrm{yr}$ (Cumming, 2002). Calculations allowing for changes in conductivity with temperature in the outer layers of a WD yield decay time scales for the dipole $(l=0)$ mode of $8-12 \times 10^{9} \mathrm{yrs}$ and for the quadrupole $(l=2)$ mode of $4-6 \times 10^{9} \mathrm{yr}$ for WDs masses in the range $0.6-1 \mathrm{M}_{\odot}$ and Carbon-Oxygen interiors (Cumming, 2002; Wendell et al., 1987). The non-linear coupling between the different modes that occurs when the Hall current is included could result in the growth of higher order modes relative to the dipole mode (Muslimov et al., 1995). However, there are estimates that have questioned whether this mechanism will give rise to the development of a dominant quadrupolar component in a typical cooling time (Cumming, 2002).

According to current observations (see Fig.18), there seems to be no evidence for a change in the incidence of magnetism or in field evolution as shown by Ferrario et al. (2015a). That is, the mean field strength and distribution about this mean seem to be independent of effective temperature. They also show that the cumulative distribution function of the effective temperatures is smooth over the full range of temperatures, again suggesting that the incidence of magnetism has remained the same throughout the life of the Galaxy. The lack of evidence for the evolution of field strength with effective temperature is consistent with the long decay time scales outlined above. However, the observational findings may not give us the full picture because the current sample of MWDs, by being Sloan-dominated, is very biased, favouring the detection of hot WDs with fields larger than a few MegaGauss. On the other hand, volumelimited samples such as that of Sion et al. (2014); Holberg et al. (2016), contain too few MWDs to establish whether the incidence of magnetism is inversely 
proportional to effective temperature (as first raised by Liebert and Sion, 1979) or if there is a link between field strength and stellar age. Larger volume-limited samples comprising MWDs distributed over a wider range of field strengths and temperatures are needed to answer this long-standing question.

Definitive statements about field structure can only be made for a few well studied MWDs. However, it is clear that complex field structures can be found in both the hotter (e.g. REJ0317-853 Ferrario et al., 1997a; Vennes et al., 2003) and the cooler (e.g. WD1953-011 Maxted et al., 2000) MWDs. Furthermore, there are examples of both high field (Euchner et al., 2005), and low field (Maxted et al., 2000; Landstreet et al., 2017) MWDs that appear to exhibit a similar level of field complexity. The complexity of the field could be linked to its origin. For instance, García-Berro et al. (2012) suggested that MWDs resulting from mergers may have a more complex structure than those descending from the magnetic Ap and Bp main sequence stars (see, e.g., Braithwaite and Spruit, 2004), if the conservation of magnetic flux from the main sequence to the compact star stage is a viable evolutionary path (see 6).

\section{Magnetic interacting binaries}

High-field MWDs are the compact objects that accrete mass from a lowmass non-degenerate companion in the MCVs. These interacting magnetic binaries comprise the polars and the intermediate polars (IPs). The IPs are powered by Roche-lobe overflow and have stable and magnetically truncated accretion discs that mask the properties of the underlying MWD. In the strongly magnetic polars the coupling radius is larger than the circularisation radius so that accretion on to the MWD is not mediated by an accretion disc. Instead, matter is channelled onto the MWD's surface by magnetically confined flows (e.g., Ferrario and Wehrse, 1999). The presence of a sub-class of polars at first named "low accretion rate polars (LARPs)", where accretion occurs from the wind emanated by the companion star, was first recognised by Schmidt et al. (2005b). These objects were later identified as the possible pro- 


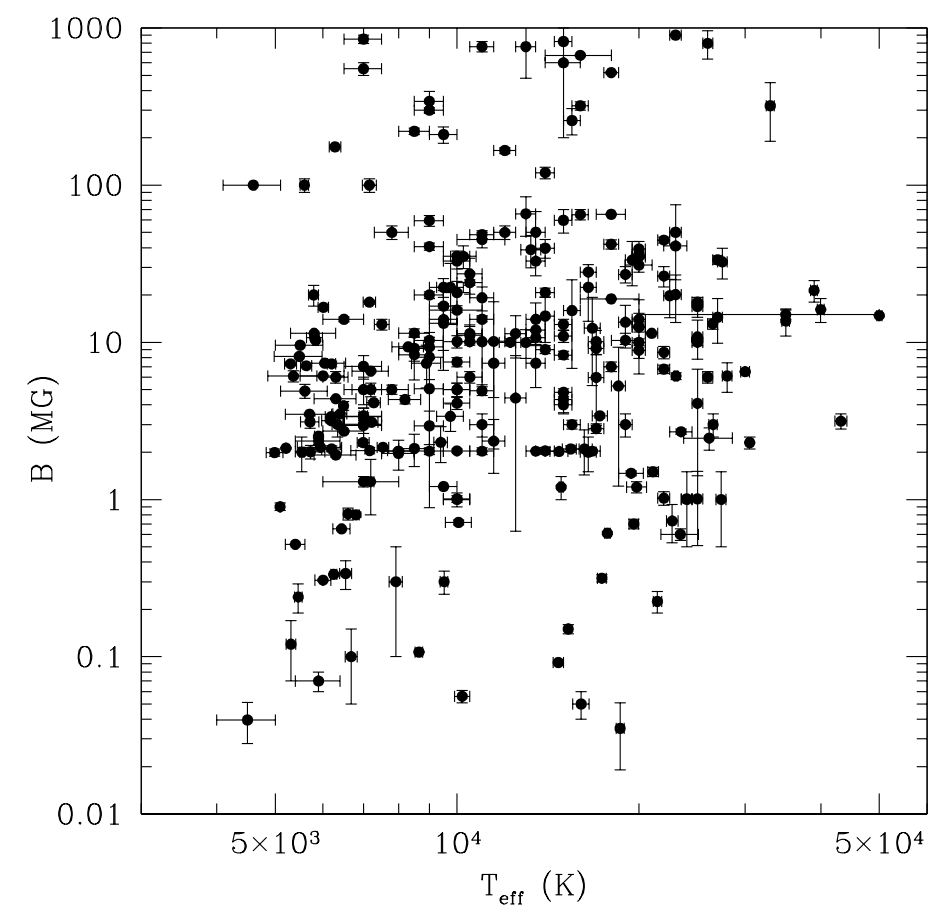

Figure 18: Magnetic field strength against effective temperature showing no indication for field evolution with cooling age (this work). 
genitors of the MCVs by Tout et al. (2008) and re-named PREPs (pre-polars) by Schwope et al. (2009) to avoid confusion (see $\S[$ ). In the polars the spin of
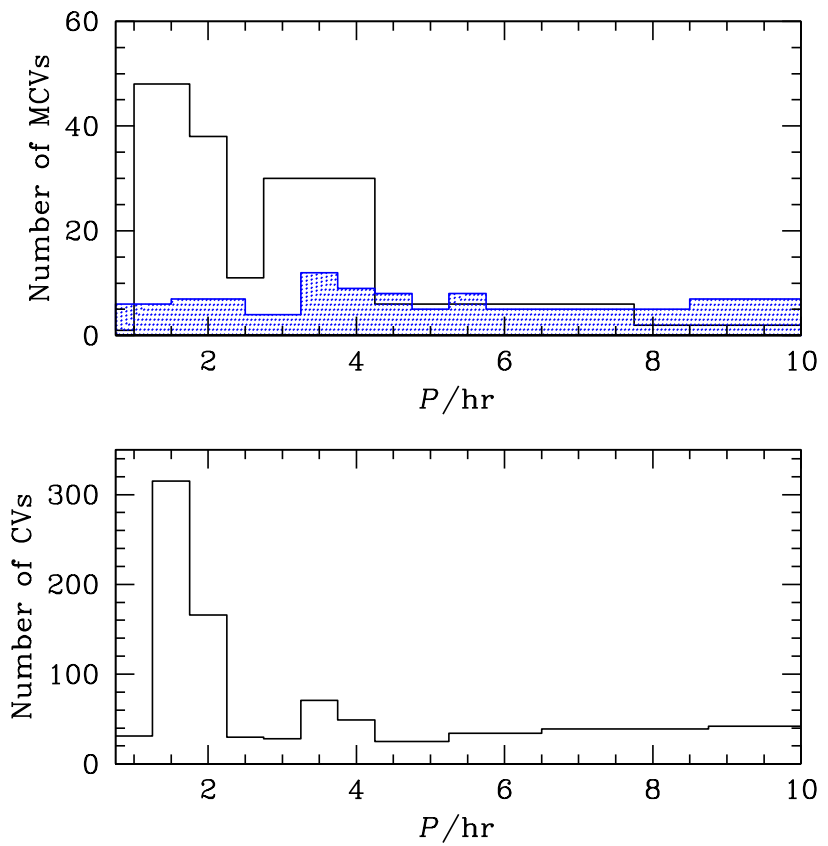

Figure 19: Top: Orbital period distribution of MCVs. The polars are depicted with the black line histogram and the IPs with the shaded histogram. Bottom: Orbital period distribution of CVs taken from Ritter and Kolb (2003). This figure is from Briggs et al. (2018b)).

the MWD is synchronised with the orbital period of the binary with the exception of a few systems, such as V1500 Cyg, BY Cam, V1432 Aql, V4633 Sgr, CD Ind, Paloma, IGR J19552+0044, and RX J0838-2827. Generally, the two periods differ by less than $2 \%$ but in two systems, Paloma (Schwarz et al., 2007) and IGR J19552+0044 (Tovmassian et al., 2017) the desynchronisation is found to be somewhat larger $(\approx 3 \%)$. This asynchronicity has been attributed to recent nova explosions given that V1500 Cyg, did undergo a nova eruption in 1975. Such systems are expected to reach synchronism over very short time-scales and Harrison and Campbell (2016) have reported that V1500 Cyg has already become fully synchronized and exhibits an X-ray spectrum and luminosity that 
are consistent with those of a polar in a high state of accretion.

The period distribution of the MCVs tends to be dominated by the polars at short orbital periods and the IPs at longer periods (see Fig.(19). Although the field strengths have been measured for only a handful of IPs, they generally seem to possess lower fields than the polars (see Fig.201). It is however very likely that some of the most strongly magnetic IPs $\left(B \gtrsim 10^{7} \mathrm{G}\right)$ may eventually evolve into polars.

Although the evolution of magnetic and non-magnetic CVs is expected to be similar, the calculations of $\mathrm{Li}$ et al. (1994) showed that magnetic braking is not as effective in strongly magnetic CVs because the magnetosphere of the MWD traps the companion star's wind. This hampers the angular momentum loss and mass transfer and leads to longer evolutionary timescales thus explaining why the fraction of MCVs among CVs is very high $(\sim 20-25 \%)$.

The polars exhibit high and low states of accretion and because they do not have a readily supply of material stored in an accretion disk, their shift from high to low states is quite sudden. During low states, the bare surface of the MWD can be observed and its magnetic field studied using the methods highlighted in $\S 2.1$. None of the known IPs has ever been observed in a low state of accretion and this is one of the reasons why it is so difficult to determine the magnetic field distribution (and structure) of the MWDs in the IPs. The orbital periods of polars are typically in the range $P_{\text {orb }} \sim 80 \mathrm{~min}$ to $8 \mathrm{hrs}$ and ideally suited for phase-resolved spectropolarimetric studies of the type already discussed for the isolated MWDs in $\S 4.7$ The magnetic properties of the accreting MWD can also be studied from the cyclotron spectra arising from the accretion shocks that occur where the material that is channeled along field lines impacts on the stellar surface (usually close to the magnetic poles). This is the only method that can be used to estimate the fields of the most strongly magnetic and polarised IPs. In all other IPs the radiation emanating from the accretion shocks is hidden in the glare of the radiation emitted by their accretion disc and the region where the stream leaving the inner Lagrangian point impacts onto the disc (the so-called "hot spot"). 


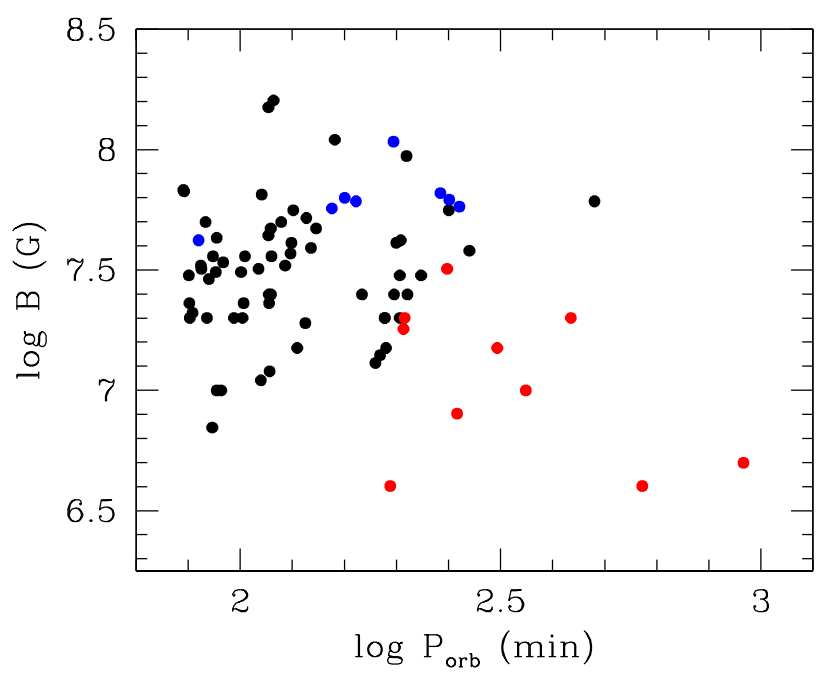

Figure 20: Magnetic field strengths of MCVs against their orbital periods. The polars are in black, the PREPs in blue and the IPs in red.

\subsection{Field structure of the MWDs in MCVs}

In this section, we focus on our understanding of the magnetic field structure of the MWDs in accreting binaries. The MWDs in the three polars EF Eri, BL Hyi and CP Tuc that have been extensively studied using Zeeman tomography (Beuermann et al., 2007, see Fig.21), exhibit field structures that are at least as complex as those in the two isolated high-field MWDs discussed in $\S 3.2$ using the same method. The additional constraints provided by the locations of the accretion shocks are also generally consistent with field structures that have strong contributions from multipoles higher than the dipole (see below). The study of the polarised radiation arising from the accretion shocks provides an excellent method to investigate the field strength and structure of the MWD in the MCVs. In these systems, the material that is channelled from the orbital plane to the surface of the MWD forms stand-off shocks that achieve temperatures

$$
T=3.7 \times 10^{8}\left(\frac{M_{\mathrm{wd}}}{M_{\odot}}\right)\left(\frac{10^{9}}{R_{\mathrm{wd}}}\right) \mathrm{K} .
$$



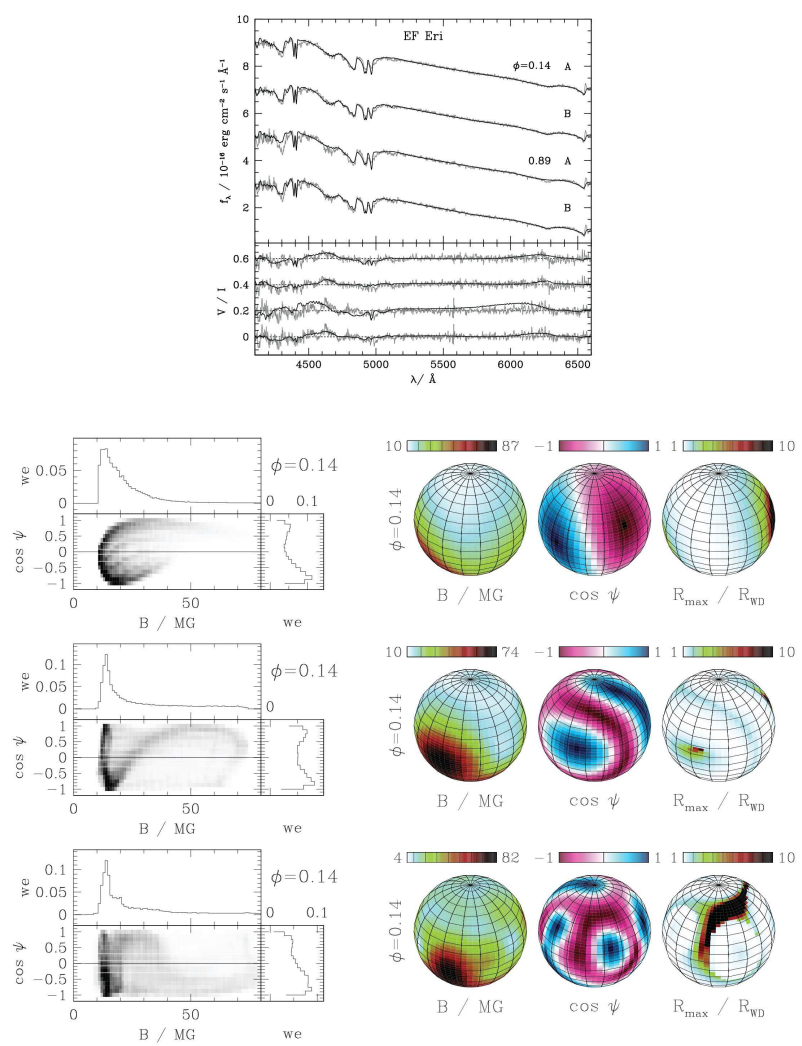

Figure 21: Top: Flux and circular polarisation spectra of EF Eri at two orbital phases for an offset dipole model (marked A) and a multipole expansion model (marked B). The black curves are the best fit models superimposed to the observed spectra in grey (see Beuermann et al., 2007, for further details). Bottom: The $B-\psi$ diagrams, where $\psi$ is the angle between the local field direction and the line of sight, corresponding to an offset dipole (top), the full multipole expansion up to $l_{\max }=3$ (centre), and the full multipole expansion up to $l_{\max }=5$ (bottom). The weight distributions (we) are also shown. The right panels show the inferred distributions of the field strength $B, \cos \psi=B_{l} / B$ (where $B_{l}$ is the field component along the line of sight) and of the maximum radius $R_{\max }$ to which the field lines extend (see Beuermann et al., 2007, for further details) 
The shocked material emits bremsstrahlung radiation in the hard X-rays and cyclotron radiation in the UV to IR spectral regions (depending on field strength). At high accretion rates and/or low fields the shock is mostly bremsstrahlung dominated. At low accretion rates and/or high fields the main emission mechanism is thermal cyclotron caused by electrons gyrating in the WD's magnetic field. The observed soft X-ray component arises from the heated photosphere of the WD near the accretion shocks (Lamb and Masters, 1979). However, some polars appear to display some puzzling anomaly whereby their soft X-ray luminosity, $L_{\text {soft }}$, is much larger than expected from simple photospheric heating caused by the re-processing of the two primary, $L_{\mathrm{brems}}$ and $L_{\mathrm{cvcl}}$, sources of radiation. That is, $L_{\text {soft }}>>L_{\text {brems }}+L_{\text {cycl }}$. Kuijpers and Pringle (1982) suggested that dense clumps of material penetrate into the photosphere of the WD to large optical depths so that the shock becomes buried. Under this condition the energy is emitted as black body radiation at a temperature much larger than the effective temperature of the WD. It has become apparent through the study of accretion processes in polars that MHD and other instabilities occurring at the magnetospheric boundary, and that are not usually encapsulated in large scale MHD simulations of accretion flows, determine the nature of field channelled flow onto the MWD.

Two possible closely related scenarios may explain why the accretion in some polars should be clump-dominated (as inferred from their soft X-ray excess) whilst in others it is not. In the first scenario (scenario 1) a strong shock forms at the stream-magnetosphere boundary. Various interchange instabilities, such as the magnetic Rayleigh-Taylor instability in the cooled post-shock region, result in the stream fragmenting into clumps of gas with length scales and masses that are set by the strength of the field. As this material penetrates the magnetosphere the initially less massive clumps couple on to field lines and feed the main accretion pole (that is, the stronger accretor) while the more massive clumps penetrate further down stream and are shredded into smaller blobs by the Kelvin-Helmholtz instability which then couple on to field lines and feed the second pole (the weaker accretor). This model is consistent with observations of 


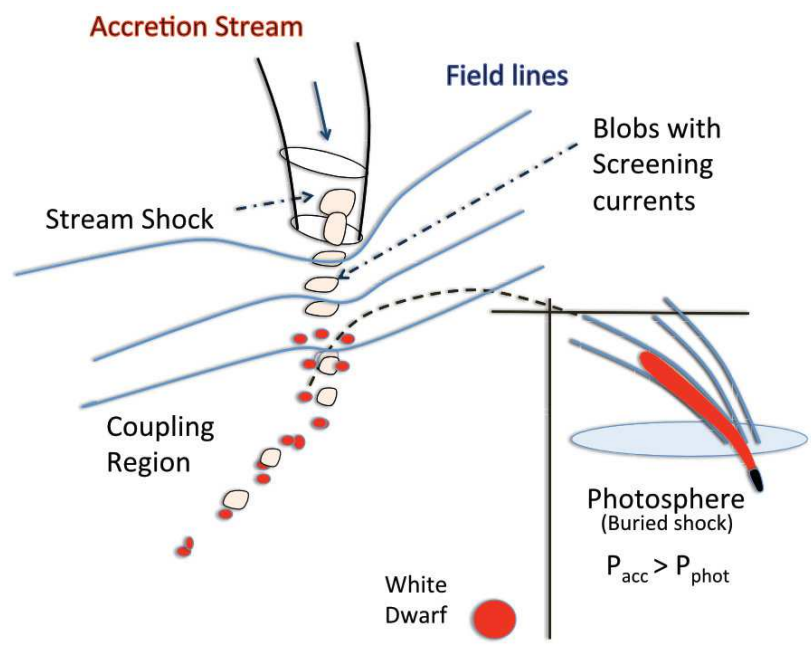

Figure 22: Schematic diagram explaining scenario 1 (see text). The shock generated at the region of impact of the stream on to the MWD's magnetosphere breaks up the material into different mass blobs because of the Rayleigh-Taylor instability limited by the strength of the magnetic field. The more massive blobs follow a dynamical trajectory but are gradually stripped of matter by the Kelvin-Helmholtz instability. The clumps get pulled and form discrete filaments as they free fall along field lines. At high specific accretion rates $\left(\gtrsim 10^{2} \mathrm{~g} \mathrm{~cm}^{-2}\right)$ the resulting shock is buried in the MWD's atmosphere and the accretion energy is released as a reprocessed soft-X-ray black-body component (this figure is from Wickramasinghe, 2014a). 
systems for which there is direct evidence for X-ray emission at $\sim 0.5 \mathrm{keV}$ from a strong stream shock but perhaps not for other systems where evidence for such emission has not been reported. We show in Fig.22 a schematic diagram depicting this scenario.

In an alternative but closely related scenario (scenario 2) the stream is continuously decelerated by a series of weak shocks as it plunges into the magnetosphere. MHD and other instabilities at its surface shred the stream into small enough mass components that diffuse into co-rotating field lines and deplete the mass of the stream until it is exhausted. This scenario is similar to what may be in operation in the inner transition region in an accretion disc where material is similarly depleted from its surface layers until the disc material is exhausted. However to date there has been no reported evidence for blobby accretion in the IPs. The physics of the stream-magnetosphere interaction has been described in more detail in the review of Wickramasinghe (2014a).

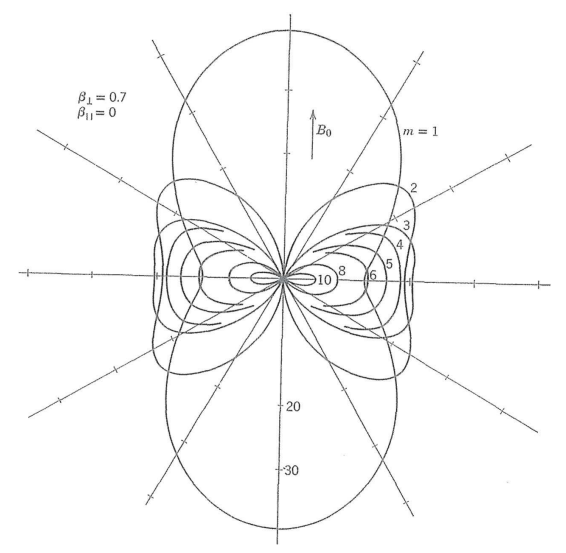

Figure 23: Angular distribution of cyclotron radiation from a mildly relativistic electron (after Oster, 1960). Here $B_{0}$ is the magnetic field direction and $m$ the cyclotron harmonic number. The curves labelled $m=1,2,3 \cdots$ enclose the volume of the beam of radiation emitted by the electron and show that as the harmonic number increases, the radiation is concentrated to an increasingly narrower cone whose axis is perpendicular to the direction of the magnetic field.

Cyclotron radiation is strongly polarised and beamed orthogonally to the magnetic field direction at harmonics numbers $m>1$ as shown in Fig.23, If the 
line of sight of a distant observer forms an angle $i$ to the spin axis of the MWD and the dipole magnetic axis is inclined by an angle $\beta$ to the rotation axis, then as the MWD rotates the angle $\psi$ between the line of sight and the field direction at the emission region varies as a function of the orbital phase $\phi$. Because cyclotron radiation is beamed, the observer will see strongly modulated intensity and polarisation curves (Meggitt and Wickramasinghe, 1982). All polars, together with a handful of IPs, exhibit a very high level of circular polarisation. As an example, we show in Fig.24 the circular polarisation curves in the $\mathrm{H}$ and $\mathrm{J}$ bands of the polar V834 Cen. Because thermal broadening dominates at most

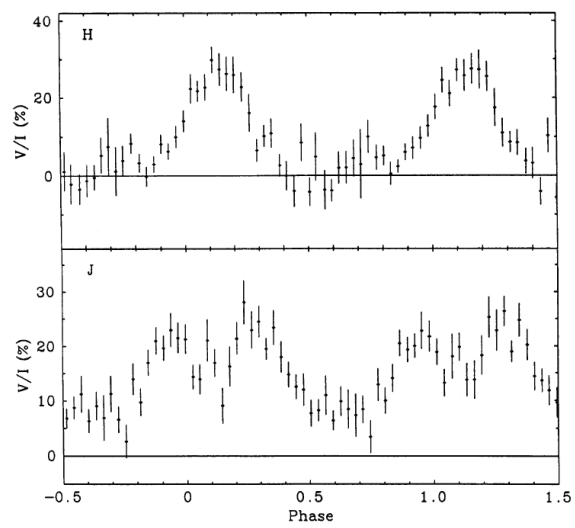

Figure 24: Circular polarisation curves of V834 Cen in the $\mathrm{H}$ and $\mathrm{J}$ infrared bands (figure from Ferrario et al., 1992)

viewing angles, cyclotron radiation generally appears as continuum emission. Nonetheless, if the shock's electron temperature is sufficiently low to prevent relativistic mass broadening to dominate, then resolvable cyclotron emission lines may become visible at those orbital phases when the magnetic field lines are nearly orthogonal to the line of sight (Meggitt and Wickramasinghe, 1982). If the accretion rate is high enough, the magnetically channelled material can flow to both foot points of closed field lines (that is, not only to the most favourably inclined pole). Thus it may be possible to detect two sets of cyclotron lines and the separation of consecutive harmonic bumps provides us with a precise mea- 
sure of the field strength at both shocks (above and below the orbital plane). At low temperatures the position of the $\mathrm{m}^{\text {th }}$ harmonic for a magnetic field $B$ and viewing angle $\psi=90^{\circ}$ to the magnetic field direction is given by:

$$
\lambda_{m}=\frac{10710}{m}\left(\frac{10^{8} \mathrm{G}}{B}\right)
$$

Cyclotron spectroscopy has proved to be a very powerful method to gain information on the magnetic field strength and structure of the MWD in MCVs as first shown by Visvanathan and Wickramasinghe (1979). As an example, we show in Fig 26] some spectra of VV Puppis from Mason et al. (2007) exhibiting two sets of prominent cyclotron harmonic features showing that the MWD was accreting near both magnetic poles. During the bright orbital phase the spectra

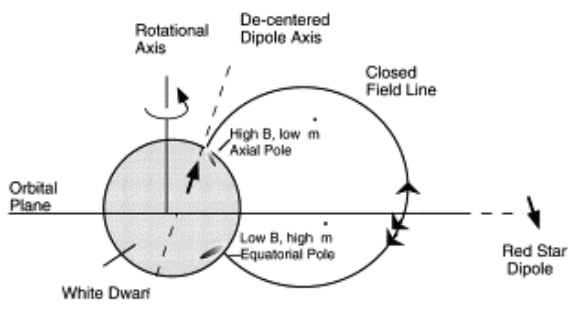

Figure 25: Schematic diagram of a MWD that accretes matter on to two regions. The more favourably inclined, and thus more strongly accreting region, is that located below the orbital plane. This region has a weaker magnetic field than the second located above the orbital plane.

show the presence of a field of $31.5 \mathrm{MG}$ (harmonic numbers 5, 6, and 7) while during the faint phase a second set of harmonics becomes visible revealing a much stronger field of 54.6 MG (harmonic numbers 3 and 4). The Zeeman absorption features, also present in the spectra of VV Pup, have been modelled as arising either from an accretion halo surrounding the cyclotron emission regions or from the photosphere of the MWD. Neither of these explanations has been satisfactory thus indicating that the field geometry of VV Pup is likely to be much more complex than can be modelled with a simple centred or off-centred dipole, as in the case for EF Eri (see above and Ferrario et al., 1996). We show 
in Fig.25 a schematic diagram of a MWD with an off-centred dipole that is accreting material on to two regions. In this figure the most favourably oriented region is that below the orbital plane. To date, we have been able to determine the field strengths of most polars through either the modelling of Zeeman split lines, or cyclotron spectroscopy, or in some cases both. Cyclotron harmonics have been detected in the IR (e.g., Bailev et al., 1991; Ferrario et al., 1993a), in the optical (Visvanathan and Wickramasinghe, 1979; Schwope and Beuermann, 1990; Ferrario et al., 1994; Mason et al., 2007) and in the most strongly magnetic systems also in the UV (e.g., Rosen et al., 2001; Gänsicke et al., 2001). It is possible that accretion may modify the field strength and/or structure
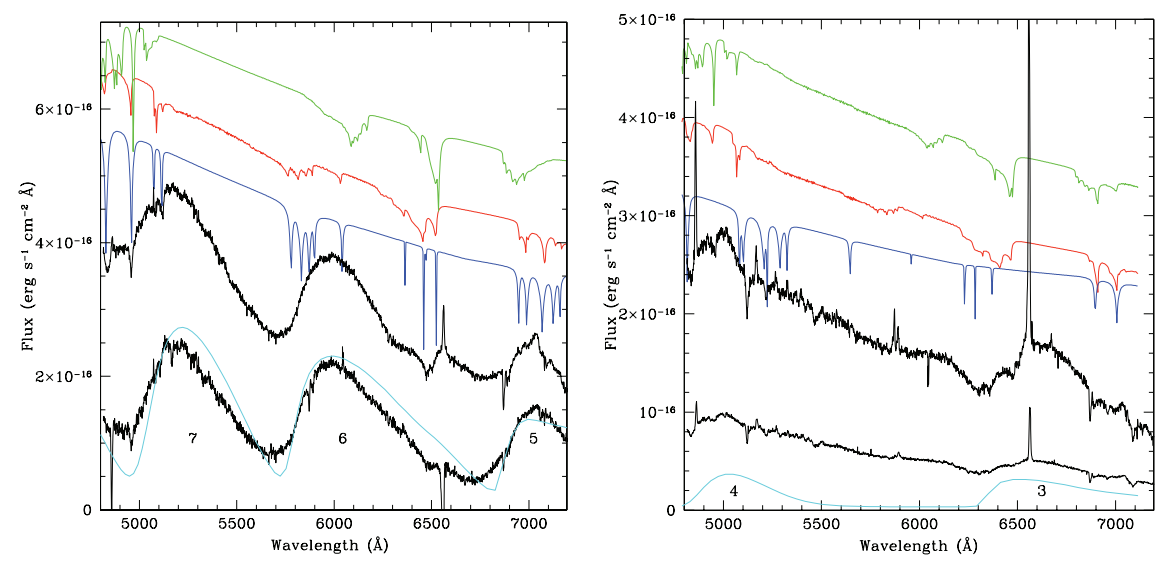

Figure 26: Left panel: Observed spectra of VV Pup (black curves). The upper spectrum is the average of all bright phase spectra of VVPup while the lower spectrum is obtained by subtracting the average faint phase spectra from the average bright phase spectra. The cyclotron model (cyan line) is overlapped to the spectrum at the bottom and the numbers denote the cyclotron harmonics which correspond to a field of 31.5 MG. Right panel: Two faint phase spectra of VV Pup (black lines) taken at two different epochs with overlapped the cyclotron model (cyan line) which correspond to a field of 54.6 MG (the numbers denote the cyclotron harmonics). Halo Zeeman features (blue lines), and photospheric Zeeman models (off-centre dipole in green and centred dipole in red) are also shown at the top of each panel. All details of observations and modelling can be found in Mason et al. (2007).

through accretion induced field decay (Cumming, 2002). The calculations of 
Zhang et al. (2009) indicate that magnetically confined flows that impact on to the surface of the MWD near the foot points of closed field lines, can advect the field toward the equator (see Fig 27) where it becomes buried. This leads

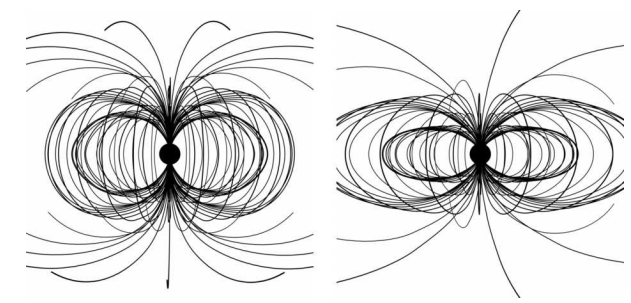

Figure 27: Schematic diagram of a dipole (left) and an advected dipole (right).

to a reduction in the polar field strength down to a bottom field of just a few $10^{3} \mathrm{G}$, regardless of the initial field strength. This mechanism was first invoked by Zhang and Kojima (2006) to explain the low fields of the millisecond radio pulsars that have undergone recycling via accretion from a companion star. However, if the total mass accreted is less than about $0.1-0.15 \mathrm{M}_{\odot}$, then the field diffuses faster than can be advected and is not modified by accretion. The mass accretion rates in polars, generally less than about $10^{16} \mathrm{~g} \mathrm{~s}^{-1}$, is low enough to prevent field restructuring. In the IPs, however, the much higher accretion rates could in principle cause significant field restructuring and decay. On the other hand, mass-loss caused by nova explosions (not present in neutron stars) could hinder the accumulation of mass onto the MWD and thus not even highly accreting IPs may suffer from field restructuring decay. Systems that have reached the bottom field are predicted to exhibit a substantially non-dipolar magnetic field structure and could be found among those dwarf novae with quasi-periodic oscillations (Zhang et al., 2009). Field screening in the IPs during phases of accretion from a disc has also been proposed by Webbink and Wickramasinghe (2002) and Cumming (2002).

Accretion in MCVs occurs via magnetically confined funnels in polars and accretion disc plus magnetically confined curtains in IPs (see, e.g., Ferrario et al., 1993b). However, the IPs FO Aqr, TX Col, BG CMi, AO Psc, and V1223 Sgr 
exhibit characteristics of both types, that is, while most of the accretion flow is mediated by an accretion disc, part of the flow appears to run over the disc to thread directly onto the magnetic field lines (e.g., see the work of Hellier (1993) on the IP FO Aqr). The IP V2400 Oph is, instead, a disc-less accretor (Buckley et al., 1995). As the star rotates around its axis a magnetically confined funnel impacts first near one of the two magnetic poles and then near the other (see Hellier et al., 1991; Ferrario and Wickramasinghe, 1999). The MCV Paloma has been proposed by Joshi et al. (2016) to also be a disc-less IP similar to V2400 Oph rather than an asynchronous polar.

Because of the radiation properties of the MCVs, the discovery of an unresolved hard (20-40 keV) X-ray emission in the inner 10 pc Galactic Centre by the Nuclear Spectroscopic Telescope Array (Perez et al.,, 2015) has been attributed to a large population of IPs (Pretorius and Mukai, 2014; Hailey et al., 2016). Non-magnetic CVs and polars, instead, may be responsible for the much softer $\mathrm{X}$-ray emission originating from the Galactic ridge (Xu et al., 2016).

\section{Origin of magnetic fields in isolated and accreting WDs}

There are two main theories for the origin of magnetic fields in WDs. The first is that the fields are essentially of fossil origin reflecting in some way the magnetic flux of their progenitor stars dating back to at least the mainsequence (Woltjer, 1964; Mestel, 1966; Moss, 2003; Tout et al., 2004). The second is that fields are generated in convective dynamos when two stars, one of which with a degenerate core, merge (see the various merging scenarios proposed by Tout et al., 2008; Nordhaus et al., 2011; García-Berro et al., 2012; Wickramasinghe et al., 2014). In the following sections we will review the work done on the origin of magnetic fields in WDs and the pros and cons of the two different generation theories noting that a full review on field generation in all stars can be found in Ferrario et al. (2015b). 


\subsection{Fossil fields}

The observed WDs are the end products of the evolution of main-sequence stars of mass $\sim 1.1-8 \mathrm{M}_{\odot}$. In this mass range, ordered large-scale magnetic fields of $\sim 300-30 \mathrm{kG}$ are observed in about $10 \%$ of early type stars (e.g., Wade et al., 2016). Their magnetic fluxes are similar to those of the MWDs at least at the high end of the field distribution suggesting a possible evolutionary link between these two groups of stars.

Additional support for a fossil origin of the fields in the high-field MWDs comes from their average mass being higher than that of their non-magnetic counterparts (see $\S 4.2$ ). This indicates that if high-field MWDs are the result of single star evolution they must, on average, descend from progenitors that are more massive $\left(\geq 2 \mathrm{M}_{\odot}\right)$ than those of non-magnetic WDs. The masses of the main-sequence Ap/Bp stars that host strong, large-scale magnetic fields would fulfill this requirement.

There are strong arguments to suggest that fields in the magnetic mainsequence stars are not generated by a contemporary dynamo but are of fossil origin. The discovery of global organised magnetic fields in about $7 \%$ of the premain sequence Herbig Ae/Be stars $\left(M \sim 2-15 \mathrm{M}_{\odot}\right)$ by Alecian et al. (2008) lends further support to this view. Calculations of pre-main-sequence stellar evolution have also shown that stars more massive than $\sim 2 \mathrm{M}_{\odot}$ are expected to reach the main sequence retaining a radiative core (Palla and Stahler, 1993$)$ in which a magnetic field may be preserved.

The numerical studies of Braithwaite and Spruit (2004) have shown that the stability of fossil fields in Ap/Bp stars require linked poloidal-toroidal field structures. Their toroidal component resides inside the star while the external field is approximately dipolar with an offset from the centre of the star much like the fields observed in $\mathrm{Ap} / \mathrm{Bp}$ stars. The subsequent evolution of such fields remains largely unexplored although various scenarios have been discussed (e.g., Tout et al., 2004; Quentin and Tout, 2018). Numerical magnetohydrodynamical calculations have shown that while the dynamo mechanism is effective in generating weak fields of the order of the equipartition value in 
the convective cores of stars with radiative envelopes (Brun et al., 2005), much stronger fields can be generated if a fossil field is present in the radiative region (Featherstone et al., 2009). It is possible that the interplay between toroidal and fossil poloidal components and the field generated in the convective core during stellar evolution may lead to a MWD's surface field structure that is much more complex than in the progenitor main-sequence star.

An argument against the fossil field hypothesis is that the birth rates of the magnetic main-sequence Ap/Bp stars are not consistent with those of the MWDs as first noted by Kawka and Vennes (2004) (see Fig.28). A better agreement can be achieved if it is assumed that in addition to the observed Ap/Bp stars, 40\% of main-sequence stars more massive than $4.5 \mathrm{M}_{\odot}$ have fields in the $10-100 \mathrm{G}$ range and also evolve into high-field MWDs (see Wickramasinghe and Ferrario, 2005, and their Figures 5 to 8 for the various proposed scenarios). However the ensuing spectropolarimetric surveys of Aurière et al. (2007) all but excluded the existence of these weak fields, revealing, instead, a "magnetic desert" below $300 \mathrm{G}$.

The arguments for and against a fossil origin of fields from single star evolution should be weighed against an important piece of evidence involving the seemingly lack of binaries consisting of a MWD with a non-degenerate and fully detached companion star (Liebert et al., 2005, 2015).

In a recent paper, Quentin and Tout (2018) follow the evolution of a $3 \mathrm{M}_{\odot}$ star with fossil toroidal and poloidal fields through the various stages of stellar evolution up to the point when a degenerate core is formed. Their calculations use a 1-D stellar evolution code modified to allow for differential rotation and use averaged large-scale poloidal and toroidal magnetic fields that evolve following the $\Omega$ and $\alpha-\Omega$ dynamo mechanisms. The models follow variations on nuclear time scales and are not equipped to allow for dynamo cycles as for instance in Brun et al. (2005) and Featherstone et al. (2009). They confirm a previous proposal by Tout et al. (2004) that large scale average fields are attenuated in the convective regions compared to radiative regions. The calculations show that a $3 \mathrm{M}_{\odot}$ star evolves into a MWD with strong toroidal fields of $10^{6}-10^{8} \mathrm{G}$ 
concentrated in its outer layers but with a very much weaker poloidal field almost independently of initial conditions. This suggests that all $3 \mathrm{M}_{\odot}$ stars (and by extrapolation all $3-8 \mathrm{M}_{\odot}$ stars) are likely to end their evolution with weak poloidal fields that will place them in the very low-field regime. The authors argue that the lower mass $\left(<3 \mathrm{M}_{\odot}\right)$ main-sequence stars with extensive convective envelopes are likely to evolve into non-magnetic WDs. The possibility that fields may be generated in stars during post main-sequence evolution in a core-envelope dynamo during helium-shell burning and later be encapsulated in the WDs resulting in low-field MWDs $\left(B \lesssim 10^{5} \mathrm{G}\right.$ ) was already proposed by, e.g., Levy and Rose (1974).

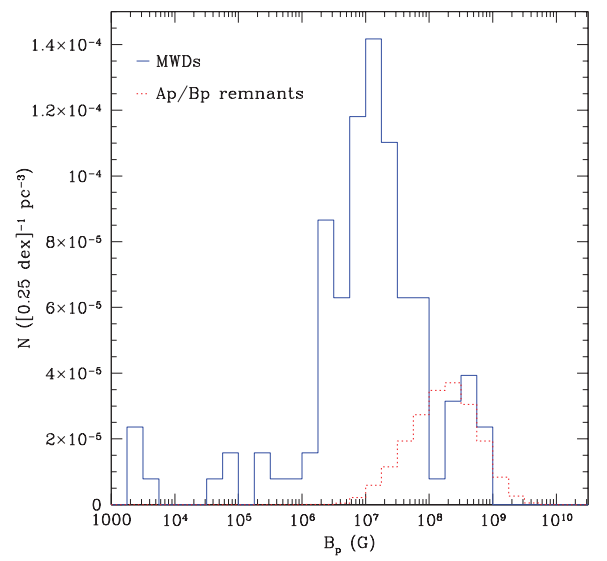

Figure 28: Solid line: incidence of magnetism among MWDs. Dotted line: predicted incidence of the remnants of magnetic Ap/Bp main-sequence stars (from Kawka and Vennes, 2004).

\subsection{High-field MWDs from common envelope mergers}

It was first noted by Liebert et al. (2005) that none of the $\sim 500$ spectroscopically observed WD+M pairs in the Sloan Digital Sky Survey (SDSS) with spectral resolutions that can measure fields greater than a few $\sim 10^{6} \mathrm{G}$ is magnetic. A follow-up study of a much larger sample of SDSS objects $(\sim 1,700)$ led to the same conclusion (Liebert et al., 2015). Likewise, of the $\sim 200$ MWDs with $B \geq 10^{6} \mathrm{G}$ that have so far been catalogued (Ferrario et al., 2015a), none has 
been found to be paired with a detached non-degenerate companion. However, we know that MWDs are commonly paired with such stars and accrete mass from them, either through Roche lobe overflow or stellar wind (see $\S 5$ ). These systems are the MCVs. Therefore, the intriguing aspect of this finding is that there are no wide binaries consisting of a MWD paired with a non-degenerate and non-interacting companion star even if wide-orbit pairs are very common among non-magnetic WDs ( 25\%, see Rebassa-Mansergas et al., 2013). This led to the suggestion that a significant proportion of the known isolated MWDs (if not all of them) may be the end product of close binary evolution as first suggested by Tout et al. (2008). The basic idea is that magnetic fields can be

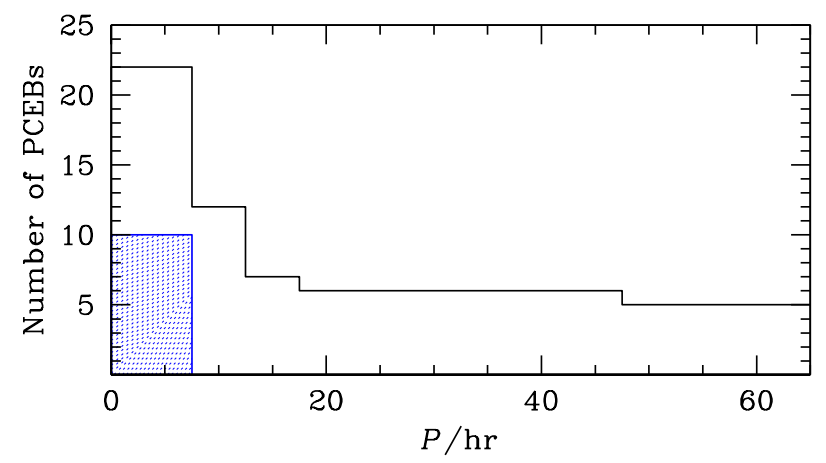

Figure 29: The orbital period distributions of PCEBs (black solid line, Nebot Gómez-Morán et al., 2013) and PREPs (shaded blue histogram).

generated in differentially rotating common envelopes (CE) and become frozen into the WDs. The strongest fields are expected in WDs that are formed when two stars merge during a CE phase of evolution (Wickramasinghe et al., 2014). The end product would be an isolated high-field MWD with a higher than average mass. Binaries whose components survive $\mathrm{CE}$ evolution and with their cores coming close enough to each other to generate a strong field in the WD, will evolve first into wind-accreting PREPs and then into bona-fide MCVs. The short orbital periods of the PREPs as compared to post-common envelope binaries (PCEBs, see Fig.29) and the high magnetic fields of PREPs (see Fig. 30) seem to support this hypothesis. If the stars emerge from $\mathrm{CE}$ with a large 
separation, they may either evolve into non-magnetic CVs or may never come into contact. There are some double degenerate binaries (DDs) consisting of a MWD with a non-magnetic WD companion (a list of these DDs can be found in Kawka et al., 2017). These could be the outcome of an initially triple system where two stars merged and generated a MWD, while the third component evolved in isolation to become a non-magnetic WD. The CE stellar interaction/merging hypothesis of Tout et al. (2008) and Wickramasinghe et al. (2014)

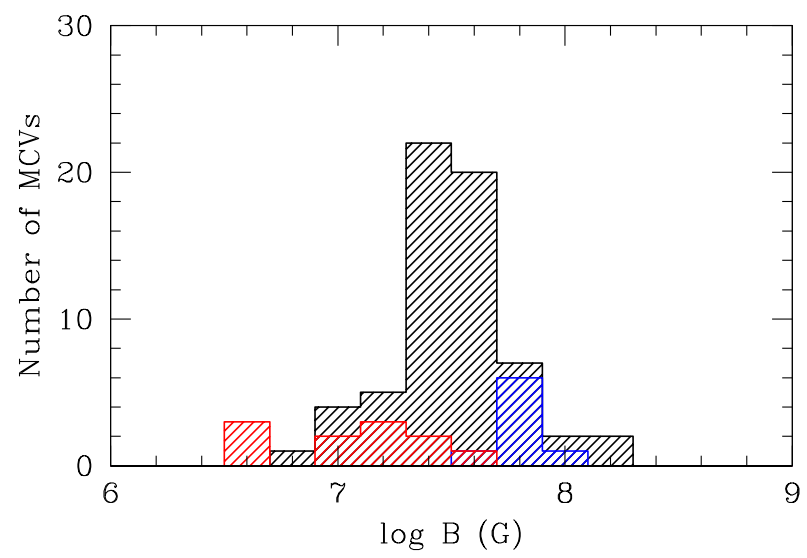

Figure 30: Magnetic field distribution of polars (black), IPs (red), and PREPs (blue).

is supported by the population synthesis calculations of Briggs et al. (2015) and Briggs et al. (2018a), in relation to the high-field MWDs, and Briggs et al. $(2018 \mathrm{~b})$, in relation to the magnetic fields of the WDs in the MCVs (but see also the work of Belloni and Schreiber, 2019). The calculations of Briggs et al. (2015) have shown that the incidence of magnetism among WDs together with their mass distribution are consistent with the view that they are the outcome of stellar merging during common envelope evolution. A follow-up study by Briggs et al. (2018a) has further demonstrated that the magnetic field strength and distribution of high-field MWDs are also consistent with the merging hypothesis. These studies were carried out by generating a population of binaries on the main-sequence and by evolving them to the age of the Galactic disc using the rapid binary stellar evolution algorithm (BSE) of Hurley et al. (2002). The 
conditions they set are that (i) as the stars enter the common envelope stage the more massive of the two (the primary) has a degenerate core in which the newly formed magnetic field can freeze-in and (ii) no further nuclear burning in the core of the star that will evolve into a WD can take place because such burning would destroy any frozen-in magnetic field. If one of these two conditions is not met the stars will evolve into non-magnetic isolated WDs or, if they have a companion, into non-magnetic binaries. In their calculations they also include double WD binaries that merge to form a single WD at any time after their last common envelope evolution. The hot DQ WDs (see section 4.4) have been proposed to be the single WD outcome of the merging of two WDs.

\section{Summary and outlook}

The magnetic fields of isolated and accreting MWDs range from $\sim 10^{3}$ to $\sim 10^{9} \mathrm{G}$ with a field distribution that peaks near a few $\times 10^{7} \mathrm{G}$. While the high-field cut-off is expected to be real, studies of the low-field distribution are compromised by the sensitivity of current spectropolarimetric surveys in the case of isolated MWDs and by the inability to observe the surface of the naked MWD in accreting binaries. In the case of accreting binaries, field strengths are either indirectly estimated through the studies of the observed accretion characteristics or, in the most strongly magnetic systems, through the detection of Zeeman and/or cyclotron lines from the MWD's surface.

The isolated MWDs appear to divide into two groups. A high-field group $\left(10^{5}-10^{9} \mathrm{G}\right)$ which dominates the current statistics because they are the easiest to discover, and a low-field group $\left(\lesssim 10^{5} \mathrm{G}\right)$, the importance of which has become apparent from spectropolarimetric surveys using $8 \mathrm{~m}$ class telescopes. Population studies of the $20 \mathrm{pc}$ volume-limited sample of MWDs suggest that at least $13 \%$ of all WDs are magnetic but the sample is incomplete at the kiloGauss level. Future spectropolarimetric surveys of even higher sensitivity may reveal that the incidence of magnetism increases at the very low field end. It is thus possible that all WDs possess a global, albeit weak, dipolar magnetic field. 
If this turns out to be the case, the accretion onto the WD in CVs may always be mediated, at least very close to the stellar surface, by the WD's magnetic field.

The tomography study of the field structure of a few isolated and accreting high-field MWDs have confirmed the presence of quite complex field structures. Other studies have indicated that complexity in field structure is not restricted to the very high field stars. Regardless of field complexity, there is so far no evidence that magnetic fields evolve with cooling age. This is consistent with theoretical estimates of the decay time-scales of the lower order dipole and quadrupole modes (4-10 Gyrs) although a larger and unbiased sample of MWDs (currently our sample is dominated by objects discovered by the SDSS) is critical to finally establish whether the incidence of magnetism is higher among cool WDs.

WDs tend in general to be slow rotators, which is evidence of efficient transfer of angular momentum from the stellar core to the envelope during pre-WD evolution (see $\S 4.3$ ). Several MWDs, however, exhibit anomalously slow rotation periods with some estimated to have periods in excess of $100 \mathrm{yrs}$. The MWDs in this group have high fields suggesting that this slow rotation may be a result of enhanced braking of the stellar core due to the presence of the magnetic field. There is also a group of high-field MWDs with a mean rotation period of a few days which may be typical for normal (non-magnetic) WDs as derived from the studies of their pulsation modes. However, some MWDs have rotation periods of less than a few hours and these could be the outcome of stellar merging or could be the end products of MCV evolution.

The significant increase in the number of known isolated and accreting MWDs has led to new insights on the nature of magnetism in these stars. However, several outstanding problems will need to be addressed before major advances can be made in this area of research. These include, for instance, the construction of more realistic model atmospheres that allow for magnetic fields. Further insights into the field structure of MWDs will require the use of more sophisticated atmospheric models combined with Zeeman tomographic 
observations extended to include all four Stokes parameters.

We conclude by noting that recent observations have shown that it is now possible to gain insights on planetary systems through the studies of the metalpolluted atmospheres of many isolated WDs. This opens up the intriguing possibility of the detection of Earth-type planets around MWDs through the discovery of the anomalous atmospheric heating or radio emission predicted by the unipolar inductor model (as it has been suggested to explain the emission lines observed in MWD GD 356), or of gaseous Jupiter-like planets through the detection of cyclotron emission from wind-driven accretion onto the MWD. The study of MCVs has also revealed the possible first discovery of a giant planet orbiting one of these systems. Thus planets and planetary systems associated to isolated and post-CE binaries may be more widespread than ever anticipated and can provide us with important clues on the formation of first and second generation planets.

Other studies have demonstrated that the polarised emission from the photospheres of MWDs could be used to place limits on fundamental physics, such as the coupling constants in some non-standard theories of gravity by measuring gravitational bi-refringence.

\section{Acknowledgements}

The authors thank Stéphane Vennes for useful discussions.

\section{References}

\section{References}

Achilleos, N., Wickramasinghe, D.T., Liebert, J., Saffer, R.A., Grauer, A.D., 1992. Exploring the peculiar magnetic field of feige 7. Astrophys. J. 396, $273-288$.

Al-Hujaj, O.A., Schmelcher, P., 2003. Electromagnetic transitions of the helium atom in superstrong magnetic fields. Phys. Rev. A 68, 053403. 
Alecian, E., Catala, C., Wade, G.A., Donati, J.F., Petit, P., Landstreet, J.D., Böhm, T., Bouret, J.C., Bagnulo, S., Folsom, C., Grunhut, J., Silvester, J., 2008. Characterization of the magnetic field of the Herbig Be star HD200775. Mon. Not. R. Astron. Soc. 385, 391-403. doi:10.1111/j.1365-2966.2008.12842.x, arXiv:0712.1746

Aurière, M., Wade, G.A., Silvester, J., Lignières, F., Bagnulo, S., Bale, K., Dintrans, B., Donati, J.F., Folsom, C.P., Gruberbauer, M., Hui Bon Hoa, A., Jeffers, S., Johnson, N., Landstreet, J.D., Lèbre, A., Lueftinger, T., Marsden, S., Mouillet, D., Naseri, S., Paletou, F., Petit, P., Power, J., Rincon, F., Strasser, S., Toqué, N., 2007. Weak magnetic fields in Ap/Bp stars. Evidence for a dipole field lower limit and a tentative interpretation of the magnetic dichotomy. Astron. Astrophys. 475, 1053-1065. doi:10.1051/0004-6361:20078189, arXiv:0710.1554,

Aznar Cuadrado, R., Jordan, S., Napiwotzki, R., Schmid, H.M., Solanki, S.K., Mathys, G., 2004. Discovery of kilogauss magnetic fields in three DA white dwarfs. Astron. Astrophys. 423, 1081-1094.

Bagnulo, S., Landstreet, J.D., 2018. Searching for the weakest detectable magnetic fields in white dwarfs. Highly-sensitive measurements from first VLT and WHT surveys. Astron. Astrophys. 618, A113-A154. doi $10.1051 / 0004-6361 / 201833235$, arXiv: 1807.09649,

Bagnulo, S., Landstreet, J.D., 2019. The long-term polarimetric variability of the strongly magnetic white dwarf Grw +70 8247. Mon. Not. R. Astron. Soc. 486, 4655-4670. doi 10.1093/mnras/stz1121, arXiv:1904.08327.

Bailey, J., Ferrario, L., Wickramasinghe, D.T., 1991. Cyclotron features in the infrared spectrum of AM Herculis. Mon. Not. R. Astron. Soc. 251, 37P-40P.

Barstow, M.A., Jordan, S., O’Donoghue, D., Burleigh, M.R., Napiwotzki, R., Harrop-Allin, M.K., 1995. RE J0317-853: the hottest known highly magnetic DA white dwarf. Mon. Not. R. Astron. Soc. 277, 971-985. 
Basri, G., Borucki, W.J., Koch, D., 2005. The Kepler Mission: A wide-field transit search for terrestrial planets [review article]. New Astron. Rev. 49, 478-485. doi $10.1016 /$ j.newar.2005.08.026

Becken, W., Schmelcher, P., 2001. Higher-angular-momentum states of the helium atom in a strong magnetic field. Phys. Rev. A 63, 053412-053424.

Becklin, E.E., Farihi, J., Jura, M., Song, I., Weinberger, A.J., Zuckerman, B., 2005. A Dusty Disk around GD 362, a White Dwarf with a Uniquely High Photospheric Metal Abundance. Astrophys. J. Lett. 632, L119-L122. doi:10.1086/497826, arXiv: astro-ph/0509193.

Belloni, D., Schreiber, M.R., 2019. Are white dwarf magnetic fields in close binaries generated during common-envelope evolution? arXiv e-prints , arXiv:1910.08582arXiv:1910.08582

Berdyugin, A.V., Piirola, V., 1999. Polarization variability in magnetic white dwarfs GD 229 and G 240-72. å 352, 619-622.

Berdyugina, S.V., Berdyugin, A.V., Piirola, V., Shapiro, A., 2007. Broad-Band Molecular Polarization in White Dwarfs, in: Napiwotzki, R., Burleigh, M.R. (Eds.), 15th European Workshop on White Dwarfs, pp. 177-182.

Bergeron, P., Dufour, P., Fontaine, G., Coutu, S., Blouin, S., Genest-Beaulieu, C., Bédard, A., Rolland, B., 2019. On the Measurement of Fundamental Parameters of White Dwarfs in the Gaia Era. Astrophys. J. 876, 67-86. doi:10.3847/1538-4357/ab153a, arXiv:1904.02022,

Beuermann, K., Buhlmann, J., Diese, J., Dreizler, S., Hessman, F.V., Husser, T.O., Miller, G.F., Nickol, N., Pons, R., Ruhr, D., Schmülling, H., Schwope, A.D., Sorge, T., Ulrichs, L., Winget, D.E., Winget, K.I., 2011. The giant planet orbiting the cataclysmic binary DP Leonis. Astron. Astrophys. 526, A53-A57. doi 10.1051/0004-6361/201015942, arXiv:1011.3905,

Beuermann, K., Euchner, F., Reinsch, K., Jordan, S., Gänsicke, B.T., 2007. Zeeman tomography of magnetic white dwarfs. IV. The complex field structure 
of the polars EF Eridani, BL Hydri and CP Tucanae. Astron. Astrophys. 463, 647-655. doi:10.1051/0004-6361:20066332, arXiv:astro-ph/0610804

Braithwaite, J., Spruit, H.C., 2004. A fossil origin for the magnetic field in A stars and white dwarfs. Nature 431, 819-821. doi 10.1038/nature02934, arXiv: astro-ph/0502043

Briggs, G.P., Ferrario, L., Tout, C.A., Wickramasinghe, D.T., 2018a. Genesis of magnetic fields in isolated white dwarfs. Mon. Not. R. Astron. Soc. 478, 899-905. doi 10.1093/mnras/sty1150, arXiv: 1804.09910

Briggs, G.P., Ferrario, L., Tout, C.A., Wickramasinghe, D.T., 2018b. Origin of magnetic fields in cataclysmic variables. Mon. Not. R. Astron. Soc. 481, 3604-3617. doi $10.1093 / \mathrm{mnras} /$ sty2481, arXiv:1809.08741

Briggs, G.P., Ferrario, L., Tout, C.A., Wickramasinghe, D.T., Hurley, J.R., 2015. Merging binary stars and the magnetic white dwarfs. Mon. Not. R. Astron. Soc. 447, 1713-1723. doi 10.1093/mnras/stu2539, arXiv:1412.5662.

Brinkworth, C.S., Burleigh, M.R., Lawrie, K., Marsh, T.R., Knigge, C., 2013. Measuring the Rotational Periods of Isolated Magnetic White Dwarfs. Astrophys. J. $773,47-62$.

Brinkworth, C.S., Burleigh, M.R., Wynn, G.A., Marsh, T.R., 2004. Photometric variability of the unique magnetic white dwarf GD 356. Mon. Not. R. Astron. Soc. 348, L33-L37.

Brinkworth, C.S., Marsh, T.R., Morales-Rueda, L., Maxted, P.F.L., Burleigh, M.R., Good, S.A., 2005. Rotational period of WD 1953-011- a magnetic white dwarf with a star-spot. Mon. Not. R. Astron. Soc. 357, 333-337.

Brun, A.S., Browning, M.K., Toomre, J., 2005. Simulations of Core Convection in Rotating A-Type Stars: Magnetic Dynamo Action. Astrophys. J. 629, 461-481. doi:10.1086/430430, arXiv:astro-ph/0610072. 
Buckley, D.A.H., Sekiguchi, K., Motch, C., O'Donoghue, D., Chen, A.L., Schwarzenberg-Czerny, A., Pietsch, W., Harrop-Allin, M.K., 1995. RX J1712.6-2414: a polarized intermediate polar from the ROSAT Galactic Plane Survey. Mon. Not. R. Astron. Soc. 275, 1028-1048.

Bues, I., Pragal, M., 1987. A new model atmosphere for G 99-37. Mem. Soc. Astron. Italiana 58, 97-100.

Burleigh, M.R., Jordan, S., Schweizer, W., 1999. Phase-resolved Far-Ultraviolet Hubble Space Telescope Spectroscopy of the Peculiar Magnetic White Dwarf RE J0317-853. Astrophys. J. Lett. 510, L37-L40.

Condon, E.U., Shortley, G.H., 1963. The theory of atomic spectra. Cambridge: Cambridge University Press.

Coutu, S., Dufour, P., Bergeron, P., Blouin, S., Loranger, E., Allard, N.F., Dunlap, B.H., 2019. Analysis of Helium-Rich White Dwarfs Polluted by Heavy Elements in the Gaia Era. arXiv e-prints, arXiv:1907.05932arXiv:1907.05932.

Cumming, A., 2002. Magnetic field evolution in accreting white dwarfs. Mon. Not. R. Astron. Soc. 333, 589-602. doi:10.1046/j.1365-8711.2002.05434.x, arXiv:astro-ph/0202079

Debes, J.H., López-Morales, M., Bonanos, A.Z., Weinberger, A.J., 2006. Detection of a Variable Infrared Excess around SDSS J121209.31+013627.7. Astrophys. J. Lett. 647, L147-L150. doi 10.1086/507486, arXiv: astro-ph/0607151.

Dufour, P., Bergeron, P., Fontaine, G., 2005. Detailed Spectroscopic and Photometric Analysis of DQ White Dwarfs. Astrophys. J. 627, 404-417.

Dufour, P., Fontaine, G., Liebert, J., Schmidt, G.D., Behara, N., 2008a. Hot DQ White Dwarfs: Something Different. Astrophys. J. 683, 978-989.

Dufour, P., Fontaine, G., Liebert, J., Williams, K., Lai, D.K., 2008b. SDSS J142625.71+575218.3: The First Pulsating White Dwarf with a Large Detectable Magnetic Field. Astrophys. J. Lett. 683, L167-L170. 
Dufour, P., Liebert, J., Fontaine, G., Behara, N., 2007. White dwarf stars with carbon atmospheres. Nature 450, 522-524.

Dufour, P., Vornanen, T., Bergeron, P., Fontaine, G., Berdyugin, A., 2013. White Dwarfs with Carbon Dominated Atmosphere: New Observations and Analysis, in: Krzesiń, ski, J., Stachowski, G., Moskalik, P., Bajan, K. (Eds.), 18th European White Dwarf Workshop., pp. 167-172.

Dunlap, B.H., Clemens, J.C., 2015. Hot DQ White Dwarf Stars as Failed Type Ia Supernovae, in: Dufour, P., Bergeron, P., Fontaine, G. (Eds.), 19th European Workshop on White Dwarfs, pp. 573-577.

Euchner, F., Jordan, S., Beuermann, K., Gänsicke, B.T., Hessman, F.V., 2002. Zeeman tomography of magnetic white dwarfs, I. Reconstruction of the field geometry from synthetic spectra. Astron. Astrophys. 390, 633-647.

Euchner, F., Jordan, S., Beuermann, K., Reinsch, K., Gänsicke, B.T., 2006. Zeeman tomography of magnetic white dwarfs. III. The 70-80 Megagauss magnetic field of PG 1015+014. Astron. Astrophys. 451, 671-681.

Euchner, F., Reinsch, K., Jordan, S., Beuermann, K., Gänsicke, B.T., 2005. Zeeman tomography of magnetic white dwarfs. ii. the quadrupole-dominated magnetic field of he 1045-0908. Astron. Astrophys. 442, 651-660.

Farihi, J., Dufour, P., Napiwotzki, R., Koester, D., 2011. The magnetic and metallic degenerate G77-50. Mon. Not. R. Astron. Soc. 413, 2559-2569.

Farihi, J., Jura, M., Zuckerman, B., 2009. Infrared Signatures of Disrupted Minor Planets at White Dwarfs. Astrophys. J. 694, 805-819. doi:10.1088/0004-637X/694/2/805, arXiv:0901.0973

Farihi, J., Koester, D., Zuckerman, B., Vican, L., Gänsicke, B.T., Smith, N., Walth, G., Breedt, E., 2016. Solar abundances of rock-forming elements, extreme oxygen and hydrogen in a young polluted white dwarf. Mon. Not. R. Astron. Soc. 463, 3186-3192. doi:10.1093/mnras/stw2182, arXiv:1608.07278. 
Farihi, J., Zuckerman, B., Becklin, E.E., 2008. Spitzer IRAC Observations of White Dwarfs. I. Warm Dust at Metal-Rich Degenerates. Astrophys. J. 674, 431-446. doi:10.1086/521715, arXiv:0710.0907.

Featherstone, N.A., Browning, M.K., Brun, A.S., Toomre, J., 2009. Effects of Fossil Magnetic Fields on Convective Core Dynamos in A-type Stars. Astrophys. J. 705, 1000-1018. doi $10.1088 / 0004-637 \mathrm{X} / 705 / 1 / 1000$.

Fendt, C., Dravins, D., 2000. Magnetic deformation of the white dwarf surface structure. Astronomische Nachrichten 321, 193-206. doi:10.1002/1521-3994(200008)321:3<193: :AID-ASNA193>3 .0.CD;2-V, arXiv:astro-ph/0007387.

Ferrario, L., 2012. Constraints on the pairing properties of main-sequence stars from observations of white dwarfs in binary systems. Mon. Not. R. Astron. Soc. 426, 2500-2506. doi $10.1111 / \mathrm{j} .1365-2966.2012 .21836 . \mathrm{x}$, arXiv:1209.1427

Ferrario, L., Bailey, J., Wickramasinghe, D., 1996. The magnetic fields of EF Eridani and BL Hydri. Mon. Not. R. Astron. Soc. 282, 218-222.

Ferrario, L., Bailey, J., Wickramasinghe, D.T., 1993a. Detection of cyclotron emission features in the infrared spectrum of ST LMi. Mon. Not. R. Astron. Soc. $262,285-288$.

Ferrario, L., de Martino, D., Gänsicke, B.T., 2015a. Magnetic White Dwarfs. Space Sci. Rev. 191, 111-169. doi 10.1007/s11214-015-0152-0, arXiv: 1504.08072

Ferrario, L., Melatos, A., Zrake, J., 2015b. Magnetic Field Generation in Stars. Space Sci. Rev. 191, 77-109. doi 10.1007/s11214-015-0138-y arXiv:1504.08074

Ferrario, L., Vennes, S., Wickramasinghe, D.T., 1998. 1RXS J0823.6-2525: a new ultramassive magnetic white dwarf. Mon. Not. R. Astron. Soc. 299, L1-L4. 
Ferrario, L., Vennes, S., Wickramasinghe, D.T., Bailey, J.A., Christian, D.J., 1997a. EUVE J0317-855 A rapidly rotating, high-field magnetic white dwarf. Mon. Not. R. Astron. Soc. 292, 205.

Ferrario, L., Wehrse, R., 1999. Accretion funnels in AM Herculis systems - I. Model characteristics. Mon. Not. R. Astron. Soc. 310, 189-202.

Ferrario, L., Wickramasinghe, D.T., 1999. The power of intermediate polars. Mon. Not. R. Astron. Soc. 309, 517-527.

Ferrario, L., Wickramasinghe, D.T., 2005. Magnetic fields and rotation in white dwarfs and neutron stars. Mon. Not. R. Astron. Soc. 356, 615-620. doi $10.1111 / j .1365-2966.2004 .08474 . x$

Ferrario, L., Wickramasinghe, D.T., Bailey, J., Hough, J.H., Tuohy, I.R., 1992. Detection of photospheric Zeeman features and cyclotron emission lines in V834 CEN in a low state. Mon. Not. R. Astron. Soc. 256, 252-260.

Ferrario, L., Wickramasinghe, D.T., Bailey, J.A., Buckley, D.A.H., 1994. The Polarization and Magnetic Field of REJ1938-461 during its Low State. Mon. Not. R. Astron. Soc. 268, 128-134.

Ferrario, L., Wickramasinghe, D.T., King, A.R., 1993b. The accretion curtain model for intermediate polars. I - A kinematical model for radial velocity and velocity dispersion. Mon. Not. R. Astron. Soc. 260, 149-162.

Ferrario, L., Wickramasinghe, D.T., Liebert, J., Schmidt, G.D., Bieging, J.H., 1997b. The magnetic field and emission-line spectrum of the remarkable white dwarf GD356. Mon. Not. R. Astron. Soc. 289, 105-116.

Fontaine, G., Brassard, P., Dufour, P., 2008. Might carbon-atmosphere white dwarfs harbour a new type of pulsating star? Astron. Astrophys. 483, L1-L4. doi $10.1051 / 0004-6361: 200809651$, arXiv:0803.2255

Forster, H., Strupat, W., Rosner, W., Wunner, G., Ruder, H., Herold, H., 1984. Hydrogen atoms in arbitrary magnetic fields. II. Bound-bound transitions. Journal of Physics B Atomic Molecular Physics 17, 1301-1319. 
Friedrich, S., Ostreicher, R., Ruder, H., Zeller, G., 1994. Line broadening in the presence of strong magnetic fields. Astron. Astrophys. 282, 179-189.

Gänsicke, B.T., Schmidt, G.D., Jordan, S., Szkody, P., 2001. Phase-resolved Hubble Space Telescope/STIS Spectroscopy of the Exposed White Dwarf in the High-Field Polar AR Ursae Majoris. Astrophys. J. 555, 380-392.

García-Berro, E., Lorén-Aguilar, P., Aznar-Siguán, G., Torres, S., Camacho, J., Althaus, L.G., Córsico, A.H., Külebi, B., Isern, J., 2012. Double Degenerate Mergers as Progenitors of High-field Magnetic White Dwarfs. Astrophys. J. 749, 25. doi 10.1088/0004-637X/749/1/25, arXiv:1202.0461.

Gentile Fusillo, N.P., Tremblay, P.E., Jordan, S., Gänsicke, B.T., Kalirai, J.S., Cummings, J., 2018. Can magnetic fields suppress convection in the atmosphere of cool white dwarfs? A case study on WD2105-820. Mon. Not. R. Astron. Soc. 473, 3693-3699. doi:10.1093/mnras/stx2584, arXiv:1710.02151.

Gnedin, Y.N., Borisov, N.V., Larionov, V.M., Natsvlishvili, T.M., Piotrovich, M.Y., Arkharov, A.A., 2006. Spectropolarimetry and IR photometry of magnetic white dwarfs: Vacuum polarization or rydberg states in their magnetic fields? Astronomy Reports 50, 553-561. doi:10.1134/S1063772906070055.

Greenstein, J.L., 1984. Spectrophotometry of the white dwarfs. Astrophys. J. 276, 602-620. doi $10.1086 / 161649$.

Greenstein, J.L., Matthews, M.S., 1957. Studies of the White Dwarfs. I. Broad Features in White Dwarf Spectra. Astrophys. J. 126, 14. doi:10.1086/146364.

Hailey, C.J., Mori, K., Perez, K., Canipe, A.M., Hong, J., Tomsick, J.A., Boggs, S.E., Christensen, F.E., Craig, W.W., Fornasini, F., Grindlay, J.E., Harrison, F.A., Nynka, M., Rahoui, F., Stern, D., Zhang, S., Zhang, W.W., 2016. Evidence for Intermediate Polars as the Origin of the Galactic Center Hard X-ray Emission. Astrophys. J. 826, 160-175. doi $10.3847 / 0004-637 X / 826 / 2 / 160$, arXiv:1605.06066 
Harrison, T.E., Campbell, R.K., 2016. The apparent synchronization of V1500 Cygni. Mon. Not. R. Astron. Soc. 459, 4161-4173. doi $10.1093 / \mathrm{mnras} / \mathrm{stw961}$

Hellier, C., 1993. Disc-overflow accretion in the intermediate polar FO Aquarii. Mon. Not. R. Astron. Soc. 265, L35-L39. doi 10.1093/mnras/265.1.L35.

Hellier, C., Cropper, M., Mason, K.O., 1991. Optical and X-ray observations of $\mathrm{AO}$ PISCIUM and the origin of the spin pulse in intermediate polars. Mon. Not. R. Astron. Soc. 248, 233-255.

Henry, R.J.W., O'Connell, R.F., 1985. Hydrogen spectrum in magnetic white dwarfs - H-alpha, H-beta and H-gamma transitions. Publ. Astron. Soc. Pac. 97, 333-339.

Hermes, J.J., Gänsicke, B.T., Kawaler, S.D., Greiss, S., Tremblay, P.E., Gentile Fusillo, N.P., Raddi, R., Fanale, S.M., Bell, K.J., Dennihy, E., Fuchs, J.T., Dunlap, B.H., Clemens, J.C., Montgomery, M.H., Winget, D.E., Chote, P., Marsh, T.R., Redfield, S., 2017. White Dwarf Rotation as a Function of Mass and a Dichotomy of Mode Line Widths: Kepler Observations of 27 Pulsating DA White Dwarfs through K2 Campaign 8. Astrophys. J. Suppl. Ser. 232, 23-50. doi $10.3847 / 1538-4365 / a a 8 b b 5$, arXiv: 1709.07004

Hoard, D.W., Schmidt, G.D., Szkody, P., Ferrario, L., Fraser, O., Wolfe, M.A., Gänsicke, B.T., 2004. The Ultraviolet Spectrum of the High-Field Magnetic Cataclysmic Variable AR Ursae Majoris. Astron. J. 128, 1894-1898.

Holberg, J.B., Oswalt, T.D., Sion, E.M., McCook, G.P., 2016. The 25 parsec local white dwarf population. Mon. Not. R. Astron. Soc. 462, 2295-2318. doi $10.1093 / \mathrm{mnras} / \mathrm{stw1357}$.

Hollands, M., Gänsicke, B., Koester, D., 2015. The incidence of magnetic fields in cool DZ white dwarfs. Mon. Not. R. Astron. Soc. 450, 681-690.

Hollands, M.A., Koester, D., Alekseev, V., Herbert, E.L., Gänsicke, B.T., 2017. Cool DZ white dwarfs - I. Identification and spectral analysis. 
Mon. Not. R. Astron. Soc. 467, 4970-5000. doi $10.1093 / \mathrm{mnras} / \mathrm{stx} 250$, arXiv:1701.07827,

Hurley, J.R., Tout, C.A., Pols, O.R., 2002. Evolution of binary stars and the effect of tides on binary populations. Mon. Not. R. Astron. Soc. 329, 897-928. doi:10.1046/j.1365-8711.2002.05038.x arXiv:astro-ph/0201220

Jordan, S., 1992. Models of white dwarfs with high magnetic fields. Astron. Astrophys. 265, 570-576.

Jordan, S., Aznar Cuadrado, R., Napiwotzki, R., Schmid, H.M., Solanki, S.K., 2007. The fraction of DA white dwarfs with kilo-Gauss magnetic fields. Astron. Astrophys. 462, 1097-1101. doi 10.1051/0004-6361:20066163, arXiv:astro-ph/0610875

Jordan, S., Friedrich, S., 2002. Search for variations in circular-polarization spectra of the magnetic white dwarf LP 790-29. Astron. Astrophys. 383, $519-523$.

Jordan, S., Schmelcher, P., Becken, W., 2001. Stationary components of He I in strong magnetic fields - a tool to identify magnetic DB white dwarfs. Astron. Astrophys. 376, 614-620.

Jordan, S., Schmelcher, P., Becken, W., Schweizer, W., 1998. Evidence for helium in the magnetic white dwarf GD 229. Astron. Astrophys. 336, L33L36.

Joshi, A., Pandey, J.C., Singh, K.P., Agrawal, P.C., 2016. PALOMA: A Magnetic CV between Polars and Intermediate Polars. Astrophys. J. 830, 56-66. doi:10.3847/0004-637X/830/2/56, arXiv:1610.00557

Jura, M., 2003. A Tidally Disrupted Asteroid around the White Dwarf G29-38. Astrophys. J. Lett. 584, L91-L94.

Jura, M., Farihi, J., Zuckerman, B., 2007. Externally Polluted White Dwarfs with Dust Disks. Astrophys. J. 663, 1285-1290. doi 10.1086/518767. arXiv:0704.1170 
Karl, C.A., Napiwotzki, R., Heber, U., Dreizler, S., Koester, D., Reid, I.N., 2005.

Rotation velocities of white dwarfs. III. DA stars with convective atmospheres.

Astron. Astrophys. 434, 637-647. doi:10.1051/0004-6361:20041437.

Kawka, A., Briggs, G.P., Vennes, S., Ferrario, L., Paunzen, E., Wickramasinghe, D.T., 2017. A fast spinning magnetic white dwarf in the double degenerate, super-Chandrasekhar system NLTT 12758. Mon. Not. R. Astron. Soc. 466, 1127-1139. doi $10.1093 / \mathrm{mnras} /$ stw3149, arXiv:1612.00325

Kawka, A., Vennes, S., 2004. Ap stars as progenitors of magnetic white dwarfs, in: Zverko, J., Ziznovsky, J., Adelman, S.J., Weiss, W.W. (Eds.), The A-Star Puzzle, pp. 879-885. doi:10.1017/S1743921305009920.

Kawka, A., Vennes, S., 2011. The cool magnetic DAZ white dwarf NLTT 10480. Astron. Astrophys. 532, A7-A14.

Kawka, A., Vennes, S., 2014. The polluted atmospheres of cool white dwarfs and the magnetic field connection. Mon. Not. R. Astron. Soc. 439, L90-L94.

Kawka, A., Vennes, S., 2016. Extreme abundance ratios in the polluted atmosphere of the cool white dwarf NLTT 19868. Mon. Not. R. Astron. Soc. 458, 325-331. doi:10.1093/mnras/stw383, arXiv:1602.05000

Kawka, A., Vennes, S., Ferrario, L., 2020. An ancient double degenerate merger in the Milky Way halo. Mon. Not. R. Astron. Soc. 491, L40-L45. doi $10.1093 / \mathrm{mnrasl} / \mathrm{slz165}$,

Kawka, A., Vennes, S., Ferrario, L., Paunzen, E., 2019. Evidence of enhanced magnetism in cool, polluted white dwarfs. Mon. Not. R. Astron. Soc. 482, 5201-5210. doi $10.1093 / \mathrm{mnras} / \mathrm{sty} 3048$ arXiv: 1811.03210

Kawka, A., Vennes, S., Schmidt, G.D., Wickramasinghe, D.T., Koch, R., 2007. Spectropolarimetric Survey of Hydrogen-rich White Dwarf Stars. Astrophys. J. 654, 499-520. 
Kemic, S.B., 1974. Wavelengths and strengths of hydrogen and helium transitions in large magnetic fields. JILA Pub. 1153.

Kilic, M., Hambly, N.C., Bergeron, P., Genest-Beaulieu, C., Rowell, N., 2018. Gaia reveals evidence for merged white dwarfs. Mon. Not. R. Astron. Soc. 479, L113-L117. doi:10.1093/mnrasl/sly110, arXiv:1805.01227.

Koester, D., Voss, B., Napiwotzki, R., Christlieb, N., Homeier, D., Lisker, T., Reimers, D., Heber, U., 2009. High-resolution UVES/VLT spectra of white dwarfs observed for the ESO SN Ia Progenitor Survey. III. DA white dwarfs. Astron. Astrophys. 505, 441-462.

Koester, D., Wilken, D., 2006. The accretion-diffusion scenario for metals in cool white dwarfs. Astron. Astrophys. 453, 1051-1057. doi:10.1051/0004-6361:20064843, arXiv: astro-ph/0603185.

Kowalski, P.M., 2010. The origin of peculiar molecular bands in cool DQ white dwarfs. Astron. Astrophys. 519, L8. doi 10.1051/0004-6361/201015238, arXiv: 1008.4542

Kuijpers, J., Pringle, J.E., 1982. Comments on radial white dwarf accretion. Astron. Astrophys. 114, L4-L6.

Kupka, F.G., Ryabchikova, T.A., Piskunov, N.E., Stempels, H.C., Weiss, W.W., 2000. VALD-2 - The New Vienna Atomic Line Database. Baltic Astronomy 9, 590-594. doi:10.1515/astro-2000-0420

Lamb, D.Q., Masters, A.R., 1979. X and UV radiation from accreting magnetic degenerate dwarfs. Astrophys. J. Lett. 234, L117-L122.

Landstreet, J.D., 1987. On the importance of the decay-induced Lorentz force to the photospheric structure of hot magnetic stars. Mon. Not. R. Astron. Soc. 225, 437-450. doi $10.1093 / \mathrm{mnras} / 225.2 .437$.

Landstreet, J.D., Bagnulo, S., 2019. Discovery of kilogauss magnetic fields on the nearby white dwarfs WD 1105-340 and WD 2150+591. Astron. Astrophys. 623, A46-A52. doi 10.1051/0004-6361/201834638, arXiv:1901.10353. 
Landstreet, J.D., Bagnulo, S., Martin, A., Valyavin, G., 2016. Discovery of an extremely weak magnetic field in the white dwarf LTT $16093=$ WD 2047+372. Astron. Astrophys. 591, A80-A85. doi:10.1051/0004-6361/201628488, arXiv:1605.04458,

Landstreet, J.D., Bagnulo, S., Valyavin, G., Valeev, A.F., 2017. Monitoring and modelling of white dwarfs with extremely weak magnetic fields. WD 2047+372 and WD 2359-434. Astron. Astrophys. 607, A92-A97. doi:10.1051/0004-6361/201731432, arXiv:1709.04099.

Landstreet, J.D., Bagnulo, S., Valyavin, G.G., Fossati, L., Jordan, S., Monin, D., Wade, G.A., 2012. On the incidence of weak magnetic fields in DA white dwarfs. Astron. Astrophys. 545, A30-A35. doi:10.1051/0004-6361/201219829, arXiv:1208.3650

Lawrie, K.A., Burleigh, M.R., Dufour, P., Hodgkin, S.T., 2013. SDSS J000555.90-100213.5: a hot, magnetic carbon-dominated atmosphere WD rotating with a 2.1 d period. Mon. Not. R. Astron. Soc. 433, 1599-1606.

Levy, E.H., Rose, W.K., 1974. Production of magnetic fields in the interiors of stars and several effects on stellar evolution. Astrophys. J. 193, 419-427. doi: $10.1086 / 153177$.

Li, J., Ferrario, L., Wickramasinghe, D., 1998. Planets around White Dwarfs. Astrophys. J. Lett. 503, L151-L154. doi 10.1086/311546.

Li, J.K., Wu, K.W., Wickramasinghe, D.T., 1994. Reduced Magnetic Braking in Synchronously Rotating Magnetic Cataclysmic Variables. Mon. Not. R. Astron. Soc. 268, 61-68. doi 10.1093/mnras/268.1.61.

Liebert, J., 1988. Searches for magnetic fields in white dwarfs since Babcock. Publ. Astron. Soc. Pac. 100, 1302-1305.

Liebert, J., Angel, J.R.P., Stockman, H.S., Beaver, E.A., 1978. Lp 790-29 a magnetic degenerate with a heavily blanketed energy distribution. Astrophys. J. 225, 181-183. 
Liebert, J., Bergeron, P., Holberg, J.B., 2003. The True Incidence of Magnetism Among Field White Dwarfs. Astron. J. 125, 348-353.

Liebert, J., Ferrario, L., Wickramasinghe, D.T., Smith, P.S., 2015. Enigmas from the Sloan Digital Sky Survey DR7 Kleinman White Dwarf Catalog. Astrophys. J. 804, 93-95. doi 10.1088/0004-637X/804/2/93, arXiv: 1504.08063

Liebert, J., Sion, E.M., 1979. Is the relative frequency of magnetic white dwarfs higher among cool stars than hot stars. Astrophys. Lett. 20, 53-55.

Liebert, J., Wickramasinghe, D.T., Schmidt, G.D., Silvestri, N.M., Hawley, S.L., Szkody, P., Ferrario, L., Webbink, R.F., Oswalt, T.D., Smith, J.A., Lemagie, M.P., 2005. Where Are the Magnetic White Dwarfs with Detached, Nondegenerate Companions? Astron. J. 129, 2376-2381. doi:10.1086/429639.

Martin, B., Wickramasinghe, D.T., 1979. Cyclotron absorption in magnetic white dwarfs. Mon. Not. R. Astron. Soc. 189, 69-77. doi:10.1093/mnras/189.1.69

Mason, E., Wickramasinghe, D., Howell, S.B., Szkody, P., 2007. First detection of Zeeman absorption lines in the polar VV Puppis. Observations of low activity states. Astron. Astrophys. 467, 277-281. doi: $10.1051 / 0004-6361: 20065354$.

Maxted, P.F.L., Ferrario, L., Marsh, T.R., Wickramasinghe, D.T., 2000. WD1953-011: a magnetic white dwarf with peculiar field structure. Mon. Not. R. Astron. Soc. 315, L41-L44.

Meggitt, S., Wickramasinghe, D., 1982. The polarization properties of magnetic accretion column. Mon. Not. R. Astron. Soc. 198, 71-82.

Merani, N., Main, J., Wunner, G., 1995. Balmer and Paschen bound-free opacities for hydrogen in strong white dwarf magnetic fields. Astron. Astrophys. 298, 193-203. 
Mestel, L., 1966. The magnetic field of a contracting gas cloud. I,Strict fluxfreezing. Mon. Not. R. Astron. Soc. 133, 265-284.

Montgomery, M.H., Williams, K.A., Winget, D.E., Dufour, P., DeGennaro, S., Liebert, J., 2008. SDSS J142625.71+575218.3: A Prototype for a New Class of Variable White Dwarf. Astrophys. J. Lett. 678, L51-L54. doi $10.1086 / 588286$, arXiv:0803.2646

Moss, D., 2003. The survival of fossil magnetic fields during pre-main sequence evolution. Astron. Astrophys. 403, 693-697. doi: $10.1051 / 0004-6361: 20030431$

Mosser, B., Goupil, M.J., Belkacem, K., Marques, J.P., Beck, P.G., Bloemen, S., De Ridder, J., Barban, C., Deheuvels, S., Elsworth, Y., Hekker, S., Kallinger, T., Ouazzani, R.M., Pinsonneault, M., Samadi, R., Stello, D., García, R.A., Klaus, T.C., Li, J., Mathur, S., Morris, R.L., 2012. Spin down of the core rotation in red giants. Astron. Astrophys. 548, A10-A23. doi:10.1051/0004-6361/201220106, arXiv:1209.3336

Musielak, Z.E., Noble, M., Porter, J.G., Winget, D.E., 2003. Chandra Observations of Magnetic White Dwarfs and Their Theoretical Implications. Astrophys. J. 593, 481-485. doi $10.1086 / 376407$.

Musielak, Z.E., Winget, D.E., Montgomery, M.H., 2005. Atmospheric Oscillations in White Dwarfs: A New Indicator of Chromospheric Activity. Astrophys. J. 630, 506-510. doi:10.1086/431790

Muslimov, A.G., van Horn, H.M., Wood, M.A., 1995. Magnetic field evolution in white dwarfs: The hall effect and complexity of the field. Astrophys. J. 442, 758-767. doi:10.1086/175481.

Nebot Gómez-Morán, A., Motch, C., Barcons, X., Carrera, F.J., Ceballos, M.T., Cropper, M., Grosso, N., Guillout, P., Hérent, O., Mateos, S., Michel, L., Osborne, J.P., Pakull, M., Pineau, F.X., Pye, J.P., Roberts, T.P., Rosen, 
S.R., Schwope, A.D., Watson, M.G., Webb, N., 2013. The XMM-Newton SSC survey of the Galactic plane. Astron. Astrophys. 553, A12-A32.

Nordhaus, J., Wellons, S., Spiegel, D.S., Metzger, B.D., Blackman, E.G., 2011. Formation of high-field magnetic white dwarfs from common envelopes. Proceedings of the National Academy of Science 108, 3135-3140. doi 10.1073/pnas.1015005108, arXiv:1010.1529,

Nousek, J.A., Shipman, H.L., Holberg, J.B., Liebert, J., Pravdo, S.H., White, N.E., Giommi, P., 1986. H $1504+65$ - an extraordinarily hot compact star devoid of hydrogen and helium. Astrophys. J. 309, 230-240. doi $10.1086 / 164594$.

O'Brien, M.S., Clemens, J.C., Kawaler, S.D., Dehner, B.T., 1996. The Pulsating Pre-White Dwarf Star PG 0122+200. Astrophys. J. 467, 397-415. doi:10.1086/177614, arXiv:astro-ph/9603025,

Oster, L., 1960. Effects of Collisions on the Cyclotron Radiation from Relativistic Particles. Phys. Rev. 119, 1444-1456.

Palla, F., Stahler, S.W., 1993. The Pre-Main-Sequence Evolution of Intermediate-Mass Stars. Astrophys. J. 418, 414-425. doi $10.1086 / 173402$.

Pelletier, C., Fontaine, G., Wesemael, F., Michaud, G., Wegner, G., 1986. Carbon pollution in helium-rich white dwarf atmospheres Timedependent calculations of the dredge-up process. Astrophys. J. 307, 242-252. doi $10.1086 / 164410$.

Perez, K., Hailey, C.J., Bauer, F.E., Krivonos, R.A., Mori, K., Baganoff, F.K., Barrière, N.M., Boggs, S.E., Christensen, F.E., Craig, W.W., Grefenstette, B.W., Grindlay, J.E., Harrison, F.A., Hong, J., Madsen, K.K., Nynka, M., Stern, D., Tomsick, J.A., Wik, D.R., Zhang, S., Zhang, W.W., Zoglauer, A., 2015. Extended hard-X-ray emission in the inner few parsecs of the Galaxy. Nature 520, 646-649. doi $10.1038 /$ nature14353 
Potter, A.T., Tout, C.A., 2010. Magnetic field evolution of white dwarfs in strongly interacting binary star systems. Mon. Not. R. Astron. Soc. 402, 1072-1080. doi 10.1111/j.1365-2966.2009.15935.x arXiv:0911.3657.

Pretorius, M.L., Mukai, K., 2014. Constraints on the space density of intermediate polars from the Swift-BAT survey. Mon. Not. R. Astron. Soc. 442, $2580-2585$.

Preuss, O., Haugan, M.P., Solanki, S.K., Jordan, S., 2004. An astronomical search for evidence of new physics: Limits on gravity-induced birefringence from the magnetic white dwarf REJ0317-853. Phys. Rev. D 70, 1-4. doi:10.1103/PhysRevD.70.067101, arXiv:gr-qc/0405068.

Putney, A., 1997. Surveying DC White Dwarfs for Magnetic Fields. Astrophys. J. Suppl. Ser. 112, 527-556.

Quentin, L.G., Tout, C.A., 2018. Rotation and magnetism in intermediate-mass stars. Mon. Not. R. Astron. Soc. 477, 2298-2309. doi:10.1093/mnras/sty770 arXiv: 1803.08270

Rebassa-Mansergas, A., Agurto-Gangas, C., Schreiber, M.R., Gänsicke, B.T., Koester, D., 2013. White dwarf main-sequence binaries from SDSS DR 8: unveiling the cool white dwarf population. Mon. Not. R. Astron. Soc. 433, $3398-3410$.

Reid, I.N., Liebert, J., Schmidt, G.D., 2001. Discovery of a Magnetic DZ White Dwarf with Zeeman-Split Lines of Heavy Elements. Astrophys. J. Lett. 550, L61-L63.

Ritter, H., Kolb, U., 2003. Catalogue of cataclysmic binaries, low-mass X-ray binaries and related objects (Seventh edition). Astron. Astrophys. 404, 301303.

Roesner, W., Wunner, G., Herold, H., Ruder, H., 1984. Hydrogen atoms in arbitrary magnetic fields. I - Energy levels and wavefunctions. Journal of Physics B Atomic Molecular Physics 17, 29-52. 
Rosen, S.R., Rainger, J.F., Burleigh, M.R., Mittaz, J.P.D., Buckley, D.A.H., Sirk, M.M., Lieu, R., Howell, S.B., de Martino, D., 2001. Multiwavelength monitoring of qs tel. Mon. Not. R. Astron. Soc. 322, 631-642.

Schmidt, G.D., Bergeron, P., Fegley, B., 1995. On the nature of spectral features in peculiar dq white dwarfs. Astrophys. J. 443, 274-280.

Schmidt, G.D., Harris, H.C., Liebert, J., Eisenstein, D.J., Anderson, S.F., Brinkmann, J., Hall, P.B., Harvanek, M., Hawley, S., Kleinman, S.J., Knapp, G.R., Krzesinski, J., Lamb, D.Q., Long, D., Munn, J.A., Neilsen, E.H., Newman, P.R., Nitta, A., Schlegel, D.J., Schneider, D.P., Silvestri, N.M., Smith, J.A., Snedden, S.A., Szkody, P., Vanden Berk, D., 2003. Magnetic White Dwarfs from the Sloan Digital Sky Survey: The First Data Release. Astrophys. J. 595, 1101-1113.

Schmidt, G.D., Szkody, P., Silvestri, N.M., Cushing, M.C., Liebert, J., Smith, P.S., 2005a. Discovery of a Magnetic White Dwarf/Probable Brown Dwarf Short-Period Binary. Astrophys. J. Lett. 630, L173-L176.

Schmidt, G.D., Szkody, P., Vanlandingham, K.M., Anderson, S.F., Barentine, J.C., Brewington, H.J., Hall, P.B., Harvanek, M., Kleinman, S.J., Krzesinski, J., Long, D., Margon, B., Neilsen, Jr., E.H., Newman, P.R., Nitta, A., Schneider, D.P., Snedden, S.A., 2005b. New Low Accretion Rate Magnetic Binary Systems and their Significance for the Evolution of Cataclysmic Variables. Astrophys. J. 630, 1037-1053.

Schmidt, G.D., Vennes, S., Wickramasinghe, D.T., Ferrario, L., 2001. Studies of magnetic and suspected-magnetic southern white dwarfs. Mon. Not. R. Astron. Soc. 328, 203-210.

Schwarz, R., Schwope, A., Staude, A., Rau, A., Hasinger, G., Urrutia, T., Motch, C., 2007. Paloma (RX J0524+42): the missing link in magnetic CV evolution. Astron. Astrophys. 473, 511-521. 
Schwope, A.D., Beuermann, K., 1990. Cyclotron and Zeeman spectroscopy of V834 Centauri. Astron. Astrophys. 238, 173-177.

Schwope, A.D., Nebot Gomez-Moran, A., Schreiber, M.R., Gänsicke, B.T., 2009. Post common envelope binaries from the SDSS. VI. SDSS J120615.73+510047.0: a new low accretion rate magnetic binary. Astron. Astrophys. 500, 867-872.

Sion, E.M., Holberg, J.B., Oswalt, T.D., McCook, G.P., Wasatonic, R., Myszka, J., 2014. The White Dwarfs within 25 pc of the Sun: Kinematics and Spectroscopic Subtypes. Astron. J. 147, 129-139. doi $10.1088 / 0004-6256 / 147 / 6 / 129$, arXiv: 1401.4989

Solanki, S.K., Haugan, M.P., Mann, R.B., 1999. Limits on gravity-induced depolarization of light from the white dwarf Grw +70 8247. Phys. Rev. D 59, 1-4. doi $10.1103 /$ PhysRevD.59.047101

Strittmatter, P.A., Wickramasinghe, D.T., 1971. The atmospheres of white dwarfs - III. The line spectra. Mon. Not. R. Astron. Soc. 152, 47-73. doi $10.1093 / \mathrm{mnras} / 152.1 .47$.

Thomas, J.H., Markiel, J.A., van Horn, H.M., 1995. Dynamo Generation of Magnetic Fields in White Dwarfs. Astrophys. J. 453, 403-413. doi: $10.1086 / 176400$.

Tout, C.A., Wickramasinghe, D.T., Ferrario, L., 2004. Magnetic fields in white dwarfs and stellar evolution. Mon. Not. R. Astron. Soc. 355, L13-L16.

Tout, C.A., Wickramasinghe, D.T., Liebert, J., Ferrario, L., Pringle, J.E., 2008. Binary star origin of high field magnetic white dwarfs. Mon. Not. R. Astron. Soc. $387,897-901$.

Tovmassian, G., Gonzales-Buitrago, D., Thorstensen, J., Kotze, E., Breytenbach, H., Schwope, A., Bernardini, F., Zharikov, S.V., Hernandez, M.S., Buckley, D.A.H., 2017. IGR J19552+0044: A new asynchronous short 
period polar. Filling the gap between intermediate and ordinary polars. Astron. Astrophys. 608, A36-A44. doi 10.1051/0004-6361/201731323, arXiv:1710.02126

Tovmassian, G., Gonzales-Buitrago, D., Zharikov, S., Reichart, D.E., Haislip, J.B., Ivarsen, K.M., LaCluyze, A.P., Moore, J.P., Miroshnichenko, A.S., 2016. Long-orbital-period Prepolars Containing Early K-type Donor Stars. Bottleneck Accretion Mechanism in Action. Astrophys. J. 819, 75. doi: $10.3847 / 0004-637 X / 819 / 1 / 75$ arXiv:1601.06240

Tovmassian, G., González, J.F., Hernández, M.S., Gonzales-Buitrago, D., Zharikov, S., Hernández Santisteban, J.V., 2018a. Quest for the Donor Star in the Magnetic Precataclysmic Variable V1082 Sgr. Astrophys. J. 869, 22-31. doi:10.3847/1538-4357/aaec02, arXiv:1811.08049,

Tovmassian, G., Szkody, P., Yarza, R., Kennedy, M., 2018b. K2 Study of the Magnetic Precataclysmic Variable V1082 Sagittarius. Astrophys. J. 863, 47-55. doi $10.3847 / 1538-4357 /$ aad229, arXiv: 1808.03722

Tremblay, P.E., Fontaine, G., Freytag, B., Steiner, O., Ludwig, H.G., Steffen, M., Wedemeyer, S., Brassard, P., 2015. On the Evolution of Magnetic White Dwarfs. Astrophys. J. 812, 19-30. doi 10.1088/0004-637X/812/1/19. arXiv:1509.05398.

Valyavin, G., Shulyak, D., Wade, G.A., Antonyuk, K., Zharikov, S.V., Galazutdinov, G.A., Plachinda, S., Bagnulo, S., Fox Machado, L., Alvarez, M., Clark, D.M., Lopez, J.M., Hiriart, D., Han, I., Jeon, Y.B., Zurita, C., Mujica, R., Burlakova, T., Szeifert, T., Burenkov, A., 2014. Suppression of cooling by strong magnetic fields in white dwarf stars. Nature 515, 88-91. doi $10.1038 /$ nature13836

Vanlandingham, K.M., Schmidt, G.D., Eisenstein, D.J., Harris, H.C., Anderson, S.F., Hall, P.B., Liebert, J., Schneider, D.P., Silvestri, N.M., Stinson, G.S., Wolfe, M.A., 2005. Magnetic White Dwarfs from the SDSS. II. The Second and Third Data Releases. Astron. J. 130, 734-741. 
Vauclair, G., Vauclair, S., Greenstein, J.L., 1979. The chemical evolution of white dwarf atmospheres : diffusion and accretion. Astron. Astrophys. 80, $79-96$.

Vennes, S., Schmidt, G.D., Ferrario, L., Christian, D.J., Wickramasinghe, D.T., Kawka, A., 2003. A Multiwavelength Study of the High-Field Magnetic White Dwarf EUVE J0317-85.5 (=RE J0317-853). Astrophys. J. 593, 1040-1048.

Visvanathan, N., Wickramasinghe, D.T., 1979. Absorption spectrum of the accretion column in VV Puppis. Nature 281, 47-48. doi:10.1038/281047a0.

Vornanen, T., Berdyugina, S.V., Berdyugin, A., 2013. Spectropolarimetric observations of cool DQ white dwarfs. Astron. Astrophys. 557, A38-A41.

Vornanen, T., Berdyugina, S.V., Berdyugin, A.V., Piirola, V., 2010. GJ 841B: the Second DQ White Dwarf With Polarized CH Molecular Bands. Astrophys. J. Lett. 720, L52-L55.

Wade, G.A., Neiner, C., Alecian, E., Grunhut, J.H., Petit, V., Batz, B.d., Bohlender, D.A., Cohen, D.H., Henrichs, H.F., Kochukhov, O., Landstreet, J.D., Manset, N., Martins, F., Mathis, S., Oksala, M.E., Owocki, S.P., Rivinius, T., Shultz, M.E., Sundqvist, J.O., Townsend, R.H.D., ud-Doula, A., Bouret, J.C., Braithwaite, J., Briquet, M., Carciofi, A.C., David-Uraz, A., Folsom, C.P., Fullerton, A.W., Leroy, B., Marcolino, W.L.F., Moffat, A.F.J., Nazé, Y., Louis, N.S., Aurière, M., Bagnulo, S., Bailey, J.D., Barbá, R.H., Blazère, A., Böhm, T., Catala, C., Donati, J.F., Ferrario, L., Harrington, D., Howarth, I.D., Ignace, R., Kaper, L., Lüftinger, T., Prinja, R., Vink, J.S., Weiss, W.W., Yakunin, I., 2016. The MiMeS survey of magnetism in massive stars: introduction and overview. Mon. Not. R. Astron. Soc. 456, 2-22. doi $10.1093 / \mathrm{mnras} / \mathrm{stv} 2568$, arXiv: 1511.08425 .

Webbink, R., Wickramasinghe, D., 2002. CV evolution: AM Her binaries and the period gap. Mon. Not. R. Astron. Soc. 335, 1-9. 
Weisskopf, M.C., Wu, K., Trimble, V., O’Dell, S.L., Elsner, R.F., Zavlin, V.E., Kouveliotou, C., 2007. A Chandra Search for Coronal X-Rays from the Cool White Dwarf GD 356. Astrophys. J. 657, 1026-1036. doi:10.1086/510776, arXiv:astro-ph/0609585

Wendell, C.E., van Horn, H.M., Sargent, D., 1987. Magnetic field evolution in white dwarfs. Astrophys. J. 313, 284-297. doi 10.1086/164968.

Werner, K., 2012. Central stars of planetary nebulae: The white dwarf connection, in: IAU Symposium, pp. 196-203. doi 10.1017/S1743921312010952, arXiv:1109.2391

Werner, K., Herwig, F., 2006. The Elemental Abundances in Bare Planetary Nebula Central Stars and the Shell Burning in AGB Stars. Publ. Astron. Soc. Pac. 118, 183-204. doi 10.1086/500443, arXiv:astro-ph/0512320.

Wesemael, F., Truran, J.W., 1982. Accretion of grains and element abundances in cool, helium-rich white dwarfs. Astrophys. J. 260, 807-814. doi:10.1086/160299.

West, S.C., 1989. The optical and near-infrared continuum polarization of five magnetic white dwarf stars - New observations and considerations regarding its origin. Astrophys. J. 345, 511-521. doi:10.1086/167926.

Wickramasinghe, D., 2014a. Accretion on to Magnetic White Dwarfs, in: European Physical Journal Web of Conferences, p. 03001. doi $10.1051 /$ epj conf/20136403001.

Wickramasinghe, D., 2014b. Accretion onto Magnetic White Dwarfs, in: European Physical Journal Web of Conferences, pp. 3001-3012.

Wickramasinghe, D.T., Farihi, J., Tout, C.A., Ferrario, L., Stancliffe, R.J., 2010. Does GD356 have a terrestrial planetary companion? Mon. Not. R. Astron. Soc. 404, 1984-1991. 
Wickramasinghe, D.T., Ferrario, L., 2000. Magnetism in Isolated and Binary White Dwarfs. Publ. Astron. Soc. Pac. 112, 873-924.

Wickramasinghe, D.T., Ferrario, L., 2005. The origin of the magnetic fields in white dwarfs. Mon. Not. R. Astron. Soc. 356, 1576-1582.

Wickramasinghe, D.T., Schmidt, G., Ferrario, L., Vennes, S., 2002. On the nature of the magnetic DB white dwarfs. Mon. Not. R. Astron. Soc. 332, 29-33.

Wickramasinghe, D.T., Tout, C.A., Ferrario, L., 2014. The most magnetic stars. Monthly Notices of the Royal Astronomical Society 437, 675-681.

Wickramasinghe, D.T., Tout, C.A., Ferrario, L., 2014. The most magnetic stars. Mon. Not. R. Astron. Soc. 437, 675-681. doi 10.1093/mnras/stt1910, arXiv:1310.2696

Willes, A.J., Wu, K., 2005. Radio emissions from terrestrial planets around white dwarfs. Astron. Astrophys. 432, 1091-1100. doi: $10.1051 / 0004-6361: 20040417$

Williams, K.A., Montgomery, M.H., Winget, D.E., Falcon, R.E., Bierwagen, M., 2016. Variability in Hot Carbon-dominated Atmosphere (Hot DQ) White Dwarfs: Rapid Rotation? Astrophys. J. 817, 27-35. doi: $10.3847 / 0004-637 X / 817 / 1 / 27$, arXiv: 1511.08834

Winget, D.E., Nather, R.E., Clemens, J.C., Provencal, J., Kleinman, S.J., Bradley, P.A., Wood, M.A., Claver, C.F., Frueh, M.L., Grauer, A.D., Hine, B.P., Hansen, C.J., Fontaine, G., Achilleos, N., Wickramasinghe, D.T., Marar, T.M.K., Seetha, S., Ashoka, B.N., O’Donoghue, D., Warner, B., Kurtz, D.W., Buckley, D.A., Brickhill, J., Vauclair, G., Dolez, N., Chevreton, M., Barstow, M.A., Solheim, J.E., Kanaan, A., Kepler, S.O., Henry, G.W., Kawaler, S.D., 1991. Asteroseismology of the DOV star PG 1159 - 035 with the Whole Earth Telescope. Astrophys. J. 378, 326-346. doi:10.1086/170434. 
Winget, D.E., Nather, R.E., Clemens, J.C., Provencal, J.L., Kleinman, S.J., Bradley, P.A., Claver, C.F., Dixson, J.S., Montgomery, M.H., Hansen, C.J., Hine, B.P., Birch, P., Candy, M., Marar, T.M.K., Seetha, S., Ashoka, B.N., Leibowitz, E.M., O’Donoghue, D., Warner, B., Buckley, D.A.H., Tripe, P., Vauclair, G., Dolez, N., Chevreton, M., Serre, T., Garrido, R., Kepler, S.O., Kanaan, A., Augusteijn, T., Wood, M.A., Bergeron, P., Grauer, A.D., 1994. Whole earth telescope observations of the DBV white dwarf GD 358. Astrophys. J. 430, 839-849. doi $10.1086 / 174455$.

Woltjer, L., 1964. X-Rays and Type i Supernova Remnants. Astrophys. J. 140, 1309-1313. doi:10.1086/148028.

Wunner, G., Roesner, W., Herold, H., Ruder, H., 1985. Stationary hydrogen lines in white dwarf magnetic fields and the spectrum of the magnetic degenerate GRW + 70 8247. Astron. Astrophys. 149, 102-108.

Xu, X.j., Wang, Q.D., Li, X.D., 2016. Fe Line Diagnostics of Cataclysmic Variables and Galactic Ridge X-Ray Emission. Astrophys. J. 818, 136. doi:10.3847/0004-637X/818/2/136, arXiv: 1602.05262 .

Zhang, C.M., Kojima, Y., 2006. The bottom magnetic field and magnetosphere evolution of neutron star in low-mass X-ray binary. Mon. Not. R. Astron. Soc. 366, 137-143. doi 10.1111/j.1365-2966.2005.09802.x. arXiv: astro-ph/0410248

Zhang, C.M., Wickramasinghe, D.T., Ferrario, L., 2009. Is there evidence for field restructuring or decay in accreting magnetic white dwarfs? Mon. Not. R. Astron. Soc. 397, 2208-2215. doi 10.1111/j.1365-2966.2009.15154.x arXiv:0905.4829.

Zhao, L.B., Stancil, P.C., 2006. Photoionization of hydrogen in white dwarf strength magnetic fields. Phys. Rev. A 74, 1-4. doi:10.1103/PhysRevA.74.055401. 
Zheleznyakov, V.V., Serber, A.V., 1991. Mass Loss by Magnetic Degenerate Stars. Soviet Astronomy Letters 17, 179-135.

Zuckerman, B., Koester, D., Dufour, P., Melis, C., Klein, B., Jura, M., 2011. An Aluminum/Calcium-rich, Iron-poor, White Dwarf Star: Evidence for an Extrasolar Planetary Lithosphere? Astrophys. J. 739, 101-110.

Zuckerman, B., Koester, D., Reid, I.N., Hünsch, M., 2003. Metal Lines in DA White Dwarfs. Astrophys. J. 596, 477-495. doi 10.1086/377492.

\section{Appendix}

Table1 lists three different angular momenta, Lánde factors and relative intensities of the Zeeman components for the most commonly detected element lines in WD atmospheres. The Lánde factors and relative intensities were calculated using the equations in $\S 2.1 .5$ 
Table 1: Lánde factors and relative intensities of the most detectable metals.

\begin{tabular}{|c|c|c|c|c|c|c|}
\hline \multicolumn{3}{|c|}{ Lower level } & \multicolumn{3}{|c|}{ Upper level } & \multirow[t]{2}{*}{ Rel. Int. } \\
\hline$J, L, S$ & $g$ & $m$ & $J, L, S$ & $g$ & $m$ & \\
\hline \multicolumn{7}{|c|}{$\mathrm{NaI} \lambda 5889.951$} \\
\hline \multirow[t]{7}{*}{$1 / 2,0,1 / 2$} & 2 & $-1 / 2$ & $3 / 2,1,1 / 2$ & $4 / 3$ & $-3 / 2$ & $3 / 16$ \\
\hline & & $-1 / 2$ & & & $-1 / 2$ & $1 / 4$ \\
\hline & & $1 / 2$ & & & $-1 / 2$ & $1 / 16$ \\
\hline & & $-1 / 2$ & & & $1 / 2$ & $1 / 26$ \\
\hline & & $1 / 2$ & & & $1 / 2$ & $1 / 4$ \\
\hline & & $1 / 2$ & & & $3 / 2$ & $3 / 16$ \\
\hline & & & $\mathrm{I} \lambda \mathrm{\lambda} 5895.924$ & & & \\
\hline \multirow[t]{5}{*}{$1 / 2,0,1 / 2$} & 2 & $-1 / 2$ & $1 / 2,1,-1 / 2$ & $4 / 3$ & $-1 / 2$ & $1 / 4$ \\
\hline & & $1 / 2$ & & & $-1 / 2$ & $1 / 4$ \\
\hline & & $-1 / 2$ & & & $1 / 2$ & $1 / 4$ \\
\hline & & $1 / 2$ & & & $1 / 2$ & $1 / 4$ \\
\hline & & & $\mathrm{g} \mathrm{I} \lambda 3832.304$ & & & \\
\hline \multirow[t]{9}{*}{$1,1,0$} & $3 / 2$ & -1 & $2,2,0$ & $4 / 3$ & -2 & $3 / 20$ \\
\hline & & -1 & & & -1 & $3 / 20$ \\
\hline & & 0 & & & -1 & $3 / 40$ \\
\hline & & -1 & & & 0 & $1 / 40$ \\
\hline & & 0 & & & 0 & $1 / 5$ \\
\hline & & 1 & & & 0 & $1 / 40$ \\
\hline & & 0 & & & 1 & $3 / 40$ \\
\hline & & 1 & & & 1 & $3 / 20$ \\
\hline & & 1 & & & 2 & $3 / 20$ \\
\hline
\end{tabular}


Table 1: Continued. Lánde factors and relative intensities of the most detectable metals.

\begin{tabular}{|c|c|c|c|c|c|c|}
\hline \multicolumn{3}{|c|}{ Lower level } & \multicolumn{3}{|c|}{ Upper level } & \multirow[t]{2}{*}{ Rel. Int } \\
\hline$J, L, S$ & $g$ & $m$ & $J, L, S$ & $g$ & $m$ & \\
\hline \multicolumn{7}{|c|}{$\operatorname{MgI} \lambda 3838.292$} \\
\hline \multirow[t]{16}{*}{$2,1,1$} & $5 / 3$ & -2 & $3,2,1$ & $4 / 3$ & -3 & $3 / 28$ \\
\hline & & -2 & & & -2 & $1 / 14$ \\
\hline & & -1 & & & -2 & $1 / 14$ \\
\hline & & -2 & & & -1 & $1 / 140$ \\
\hline & & -1 & & & -1 & $4 / 35$ \\
\hline & & 0 & & & -1 & $3 / 70$ \\
\hline & & -1 & & & 0 & $3 / 140$ \\
\hline & & 0 & & & 0 & $9 / 70$ \\
\hline & & 1 & & & 0 & $3 / 140$ \\
\hline & & 0 & & & 1 & $3 / 70$ \\
\hline & & 1 & & & 1 & $4 / 35$ \\
\hline & & 2 & & & 1 & $1 / 140$ \\
\hline & & 1 & & & 2 & $1 / 14$ \\
\hline & & 2 & & & 2 & $1 / 14$ \\
\hline & & 2 & & & 3 & $3 / 28$ \\
\hline & \multicolumn{6}{|c|}{ AlI $\lambda 3944.006$} \\
\hline \multirow[t]{5}{*}{$1 / 2,1,-1 / 2$} & $2 / 3$ & $-1 / 2$ & $1 / 2,0,1 / 2$ & 2 & $-1 / 2$ & $1 / 4$ \\
\hline & $2 / 3$ & $1 / 2$ & & & $-1 / 2$ & $1 / 4$ \\
\hline & $2 / 3$ & $-1 / 2$ & & & $1 / 2$ & $1 / 4$ \\
\hline & $2 / 3$ & $1 / 2$ & & & $1 / 2$ & $1 / 4$ \\
\hline & \multicolumn{6}{|c|}{ AlI $\lambda 3961.520$} \\
\hline $3 / 2,1,1 / 2$ & $4 / 3$ & $-3 / 2$ & $1 / 2,0,1 / 2$ & 2 & $-1 / 2$ & $3 / 16$ \\
\hline & & $-1 / 2$ & & & $-1 / 2$ & $1 / 4$ \\
\hline & & $1 / 2$ & & & $-1 / 2$ & $1 / 16$ \\
\hline & & $-1 / 2$ & & & $1 / 2$ & $1 / 16$ \\
\hline & & $1 / 2$ & & & $1 / 2$ & $3 / 16$ \\
\hline
\end{tabular}


Table 1: Continued. Lánde factors and relative intensities of the most detectable metals.

\begin{tabular}{|c|c|c|c|c|c|c|}
\hline \multicolumn{3}{|c|}{ Lower level } & \multicolumn{3}{|c|}{ Upper level } & \multirow[t]{2}{*}{ Rel. Int. } \\
\hline$J, L, S$ & $g$ & $m$ & $J, L, S$ & $g$ & $m$ & \\
\hline \multicolumn{7}{|c|}{ CaII $\lambda 3933.663$} \\
\hline \multirow[t]{6}{*}{$1 / 2,0,1 / 2$} & 2 & $-1 / 2$ & $3 / 2,1,1 / 2$ & $4 / 3$ & $1 / 2$ & $1 / 16$ \\
\hline & & $1 / 2$ & & & $3 / 2$ & $3 / 16$ \\
\hline & & $-1 / 2$ & & & $-1 / 2$ & $1 / 4$ \\
\hline & & $1 / 2$ & & & $1 / 2$ & $1 / 4$ \\
\hline & & $-1 / 2$ & & & $-3 / 2$ & $3 / 16$ \\
\hline & & $1 / 2$ & & & $-1 / 2$ & $1 / 16$ \\
\hline \multicolumn{7}{|c|}{ CaII $\lambda 3968.469$} \\
\hline \multirow[t]{4}{*}{$1 / 2,0,1 / 2$} & 2 & $-1 / 2$ & $1 / 2,1,1 / 2$ & $2 / 3$ & $1 / 2$ & $1 / 4$ \\
\hline & & $-1 / 2$ & & & $-1 / 2$ & $1 / 4$ \\
\hline & & $1 / 2$ & & & $1 / 2$ & $1 / 4$ \\
\hline & & $1 / 2$ & & & $-1 / 2$ & $1 / 4$ \\
\hline \multicolumn{7}{|c|}{$\mathrm{CaI} \lambda 4226.728$} \\
\hline $0,0,0$ & 0 & 0 & $1,1,0$ & 1 & 1 & $1 / 4$ \\
\hline & & 0 & & & 0 & $1 / 2$ \\
\hline & & 0 & & & -1 & $1 / 4$ \\
\hline
\end{tabular}




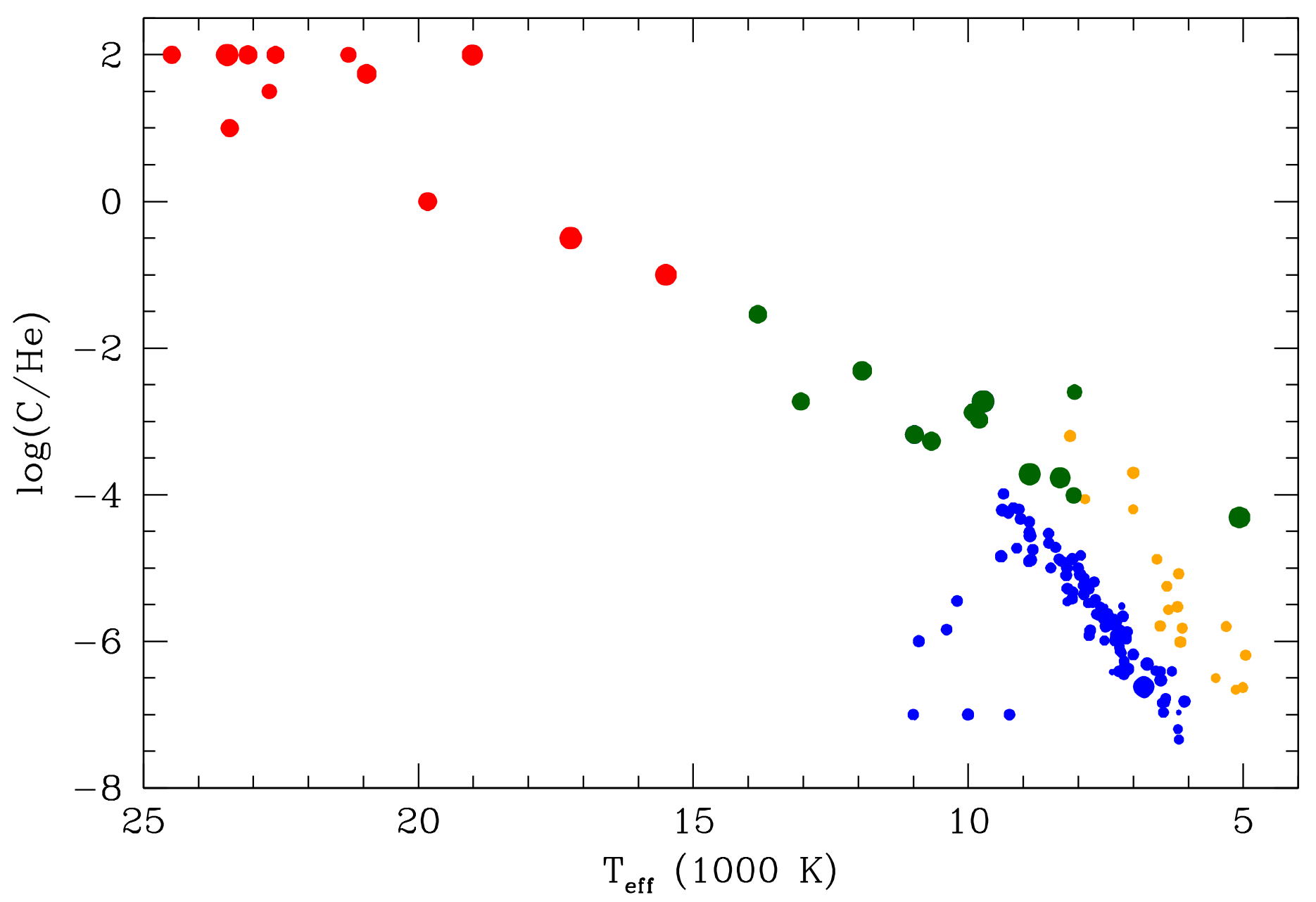



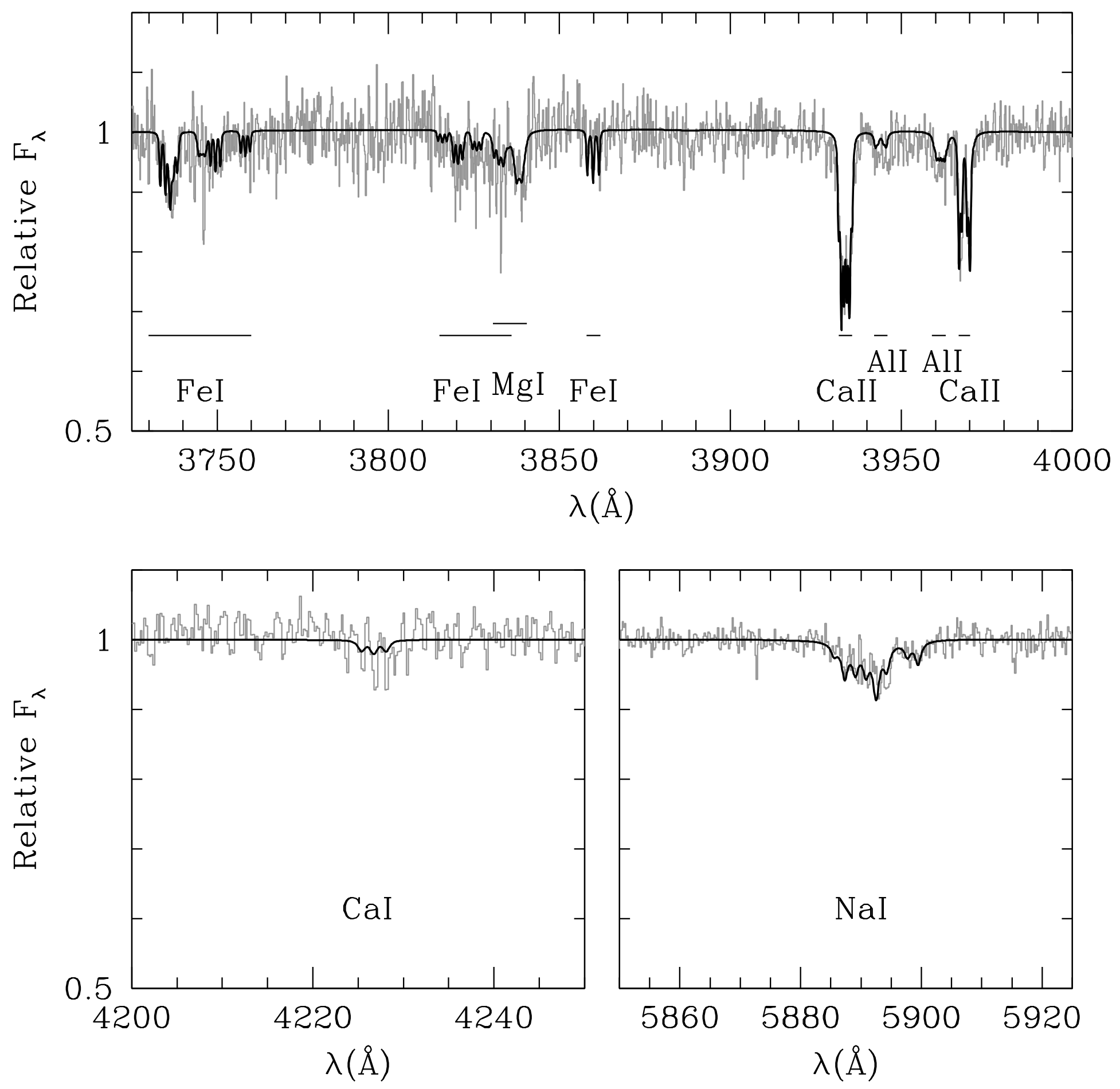